\title{
Higher arithmetic Chow groups
}

\author{
José Ignacio Burgos Gil and Elisenda Feliu*
}

\begin{abstract}
We give a new construction of higher arithmetic Chow groups for quasi-projective arithmetic varieties over a field. Our definition agrees with the higher arithmetic Chow groups defined by Goncharov for projective arithmetic varieties over a field. These groups are the analogue, in the Arakelov context, of the higher algebraic Chow groups defined by Bloch. For projective varieties the degree zero group agrees with the arithmetic Chow groups defined by Gillet and Soule, and in general with the arithmetic Chow groups of Burgos. Our new construction is shown to be a contravariant functor and is endowed with a product structure, which is commutative and associative.
\end{abstract}

Mathematics Subject Classification (2010). 14G40, 14C15, $14 \mathrm{~F} 43$.

Keywords. Arakelov geometry, higher Chow groups, Beilinson regulator, intersection theory, Deligne cohomology.

\section{Introduction}

Let $X$ be an arithmetic variety, i.e. a regular scheme which is flat and quasi-projective over an arithmetic ring. In [14], Gillet and Soule defined the arithmetic Chow groups of $X$, denoted by $\widehat{\mathrm{CH}}^{p}(X)$, whose elements are classes of pairs $\left(Z, g_{Z}\right)$, with $Z$ a codimension $p$ subvariety of $X$ and $g_{Z}$ a Green current for $Z$. Later, in [5], the first author gave an alternative definition for the arithmetic Chow groups, involving the Deligne complex of differential forms with logarithmic singularities along infinity, $\mathcal{D}_{\log }^{*}(X, p)$, that computes real Deligne-Beilinson cohomology, $H_{\mathscr{D}}^{*}(X, \mathbb{R}(p))$. When $X$ is proper, the two definitions are related by a natural isomorphism that takes into account the different normalization of both definitions. In this paper, we follow the latter definition.

It is shown in [5] that the following properties are satisfied by $\widehat{\mathrm{CH}}^{p}(X)$ :

- The groups $\widehat{\mathrm{CH}}^{p}(X)$ fit into an exact sequence:

$$
\mathrm{CH}^{p-1, p}(X) \stackrel{\rho}{\rightarrow} \mathscr{D}_{\log }^{2 p-1}(X, p) / \operatorname{im} d_{\mathscr{D}} \stackrel{a}{\rightarrow} \widehat{\mathrm{CH}}^{p}(X) \stackrel{\zeta}{\rightarrow} \mathrm{CH}^{p}(X) \rightarrow 0,
$$

\footnotetext{
*This work was partially supported by the projects MTM2006-14234-C02-01 and MTM2009-14163-C02-01.
} 
where $\mathrm{CH}^{p-1, p}(X)$ is the term $E_{2}^{p-1,-p}(X)$ of the Quillen spectral sequence (see [23], §7) and $\rho$ is the Beilinson regulator.

- There is a pairing

$$
\widehat{\mathrm{CH}}^{p}(X) \otimes \widehat{\mathrm{CH}}^{q}(X) \stackrel{\dot{\rightarrow}}{\widehat{\mathrm{CH}}^{p+q}}(X)_{\mathbb{Q}}
$$

turning $\bigoplus_{p \geq 0} \widehat{\mathrm{CH}}^{p}(X)_{\mathbb{Q}}$ into a commutative graded unitary $\mathbb{Q}$-algebra.

- If $f: X \rightarrow Y$ is a morphism, there exists a pull-back morphism

$$
f^{*}: \widehat{\mathrm{CH}}^{p}(Y) \rightarrow \widehat{\mathrm{CH}}^{p}(X) .
$$

Assume that $X$ is proper and defined over an arithmetic field. Then the arithmetic Chow groups have been extended to higher degrees by Goncharov, in [16]. These groups are denoted by $\widehat{\mathrm{CH}}^{p}(X, n)$ and are constructed in order to extend the exact sequence (1) to a long exact sequence of the form

$$
\begin{aligned}
\cdots \rightarrow & \widehat{\mathrm{CH}}^{p}(X, n) \stackrel{\zeta}{\rightarrow} \mathrm{CH}^{p}(X, n) \stackrel{\rho}{\rightarrow} H_{\mathscr{D}}^{2 p-n}(X, \mathbb{R}(p)) \stackrel{a}{\rightarrow} \widehat{\mathrm{CH}}^{p}(X, n-1) \rightarrow \cdots \\
& \cdots \rightarrow \mathrm{CH}^{p}(X, 1) \stackrel{\rho}{\rightarrow} \mathscr{D}_{\log }^{2 p-1}(X, p) / \operatorname{im} d_{\mathscr{D}} \stackrel{a}{\rightarrow} \widehat{\mathrm{CH}}^{p}(X) \stackrel{\zeta}{\rightarrow} \mathrm{CH}^{p}(X) \rightarrow 0 .
\end{aligned}
$$

Explicitly, Goncharov defined a regulator morphism

$$
Z^{p}(X, *) \stackrel{\mathcal{P}}{\rightarrow} \mathscr{D}_{D}^{2 p-*}(X, p),
$$

where

- $Z^{p}(X, *)$ is the chain complex given by Bloch in [3], whose homology groups are, by definition, $\mathrm{CH}^{p}(X, *)$;

- $\mathscr{D}_{D}^{*}(X, *)$ is the Deligne complex of currents.

Then the higher arithmetic Chow groups of a regular complex variety $X$ are defined as $\widehat{\mathrm{CH}}^{p}(X, n):=H_{n}\left(s\left(\mathcal{P}^{\prime}\right)\right)$, the homology groups of the simple of the induced morphism

$$
\mathcal{P}^{\prime}: Z^{p}(X, *) \stackrel{\mathcal{P}}{\rightarrow} \mathscr{D}_{D}^{2 p-*}(X, p) / \mathscr{D}^{2 p}(X, p) .
$$

For $n=0$, these groups agree with the ones given by Gillet and Soulé. However, this construction leaves the following questions open:

(1) Does the composition of the isomorphism $K_{n}(X)_{\mathbb{Q}} \cong \bigoplus_{p \geq 0} \mathrm{CH}^{p}(X, n)_{\mathbb{Q}}$ with the morphism induced by $\mathcal{P}$ agree with the Beilinson regulator?

(2) Can one define a product structure on $\bigoplus_{p, n} \widehat{\mathrm{CH}}^{p}(X, n)$ ?

(3) Are there well-defined pull-back morphisms? 
The use of the complex of currents in the definition of $\mathcal{P}$ is the main obstacle encountered when trying to answer these questions, since this complex does not behave well under pull-back or products. Moreover, the usual techniques for the comparison of regulators apply to morphisms defined for the class of quasi-projective varieties, which is not the case of $\mathcal{P}$.

In this paper we develop a higher arithmetic intersection theory by giving a new definition of the higher arithmetic Chow groups, based on a representative of the Beilinson regulator at the chain complex level. Our strategy has been to use the Deligne complex of differential forms instead of the Deligne complex of currents in the construction of the representative of the Beilinson regulator. The obtained regulator turns out to be a minor modification of the regulator described by Bloch in [4].

The present definition of higher arithmetic Chow groups is valid for quasi-projective arithmetic varieties over a field, pull-back morphisms are well-defined and can be given a commutative and associative product structure. Therefore, this construction overcomes the open questions left by Goncharov's construction.

The authors, jointly with Takeda, prove in [6] that this definition agrees with Goncharov's definition when the arithmetic variety is projective. Moreover, by a direct comparison of our regulator with $\mathcal{P}$, it is also proved that the regulator defined by Goncharov induces the Beilinson regulator. In this way, the open questions (1)-(3) are answered positively. Moreover, the question of the covariance of the higher arithmetic Chow groups with respect to proper morphisms will also be treated elsewhere.

Note that since the theory of higher algebraic Chow groups given by Bloch, $\mathrm{CH}^{p}(X, n)$ is only fully established for schemes over a field, we have to restrict ourselves to arithmetic varieties over a field. Therefore, the following question remains open:

(1) Can we extend the definition to arithmetic varieties over an arithmetic ring?

Let us now briefly describe the constructions presented in this paper. First, for the construction of the higher Chow groups, instead of using the simplicial complex defined by Bloch in [3], we use its cubical analog, defined by Levine in [19], due to its suitability for describing the product structure on $\mathrm{CH}^{*}(X, *)$. Thus $Z^{p}(X, n)_{0}$ will denote the normalized chain complex associated to a cubical abelian group. Let $X$ be a complex algebraic manifold. For every $p \geq 0$, we define two cochain complexes, $\mathscr{D}_{\mathbb{A}, Z^{p}}^{*}(X, p)_{0}$ and $\mathscr{D}_{\mathbb{A}}^{*}(X, p)_{0}$, constructed out of differential forms on $X \times \square^{n}$ with logarithmic singularities along infinity $\left(\square=\mathbb{P}^{1} \backslash\{1\}\right)$. For every $p \geq 0$, the following isomorphisms are satisfied:

$$
\begin{aligned}
H^{2 p-n}\left(D_{\mathbb{A}, Z^{p}}^{*}(X, p)_{0}\right) \cong \mathrm{CH}^{p}(X, n)_{\mathbb{R}}, & n \geq 0, \\
H^{r}\left(\mathscr{D}_{\mathbb{A}}^{*}(X, p)_{0}\right) \cong H_{\mathscr{D}}^{r}(X, \mathbb{R}(p)), & r \leq 2 p,
\end{aligned}
$$


where the first isomorphism is obtained by a explicit quasi-isomorphism

$$
\mathscr{D}_{\mathbb{A}, Z^{p}}^{2 p-*}(X, p)_{0} \longrightarrow Z^{p}(X, *)_{0} \otimes \mathbb{R}
$$

(see $\$ 2.4$ and $\S 2.5$ ).

We show that there is a natural chain morphism (see $\S 3.1)$

$$
\mathscr{D}_{\mathbb{A}, Z^{p}}^{2 p-*}(X, p)_{0} \stackrel{\rho}{\rightarrow} \mathscr{D}_{\mathbb{A}}^{2 p-*}(X, p)_{0}
$$

which induces, after composition with the isomorphism

$$
K_{n}(X)_{\mathbb{Q}} \cong \bigoplus_{p \geq 0} \mathrm{CH}^{p}(X, n)_{\mathbb{Q}}
$$

described by Bloch in [3], the Beilinson regulator (Theorem 3.5):

$$
K_{n}(X)_{\mathbb{Q}} \cong \bigoplus_{p \geq 0} \mathrm{CH}^{p}(X, n)_{\mathbb{Q}} \stackrel{\rho}{\rightarrow} \bigoplus_{p \geq 0} H_{\mathcal{D}}^{2 p-n}(X, \mathbb{R}(p)) .
$$

In the second part of this paper we use the morphism $\rho$ to define the higher arithmetic Chow group $\widehat{\mathrm{CH}}^{p}(X, n)$, for any arithmetic variety $X$ over a field. The formalism underlying our definition is the theory of diagrams of complexes and their associated simple complexes, developed by Beilinson in [1]. Let $X_{\Sigma}$ denote the complex manifold associated with $X$ and let $\sigma$ be the involution that acts as complex conjugation on the space and on the coefficients. As usual $\sigma$ as superscript will mean the fixed part under $\sigma$. Then one considers the diagram of chain complexes

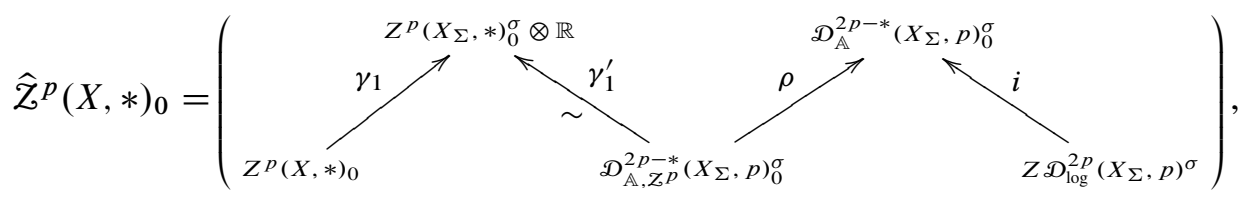

where $Z \mathscr{D}_{\log }^{2 p}\left(X_{\Sigma}, p\right)^{\sigma}$ is the group of closed elements of $\mathscr{D}_{\log }^{2 p}\left(X_{\Sigma}, p\right)^{\sigma}$ considered as a complex concentrated in degree 0 . Then the higher arithmetic Chow groups of $X$ are given by the homology groups of the simple of the diagram $\widehat{\mathcal{Z}}^{p}(X, *)_{0}$ (Definition 4.3):

$$
\widehat{\mathrm{CH}}^{p}(X, n):=H_{n}\left(s\left(\widehat{\mathcal{Z}}^{p}(X, *)_{0}\right)\right) .
$$

The following properties are shown:

- Theorem 4.8: Let $\widehat{\mathrm{CH}}^{p}(X)$ denote the arithmetic Chow group defined in [5]. Then there is a natural isomorphism

$$
\widehat{\mathrm{CH}}^{p}(X) \stackrel{\cong}{\rightrightarrows} \widehat{\mathrm{CH}}^{p}(X, 0) .
$$

It follows that if $X$ is proper, $\widehat{\mathrm{CH}}^{p}(X, 0)$ agrees with the arithmetic Chow group defined by Gillet and Soulé in [14]. 
- Proposition 4.4: There is a long exact sequence

$$
\begin{aligned}
\cdots \rightarrow \widehat{\mathrm{CH}}^{p}(X, n) \stackrel{\zeta}{\rightarrow} \mathrm{CH}^{p}(X, n) \stackrel{\rho}{\longrightarrow} H_{\mathscr{D}}^{2 p-n}\left(X_{\Sigma}, \mathbb{R}(p)\right)^{\sigma} \\
\quad \stackrel{a}{\longrightarrow} \widehat{\mathrm{CH}}^{p}(X, n-1) \rightarrow \cdots \rightarrow \mathrm{CH}^{p}(X, 1) \\
\quad \stackrel{\rho}{\longrightarrow} D_{\log }^{2 p-1}\left(X_{\Sigma}, p\right)^{\sigma} / \operatorname{im} d_{\mathscr{D}} \stackrel{a}{\longrightarrow} \widehat{\mathrm{CH}}^{p}(X) \stackrel{\zeta}{\longrightarrow} \mathrm{CH}^{p}(X) \rightarrow 0,
\end{aligned}
$$

with $\rho$ the Beilinson regulator.

- Proposition 4.12 (Pull-back): Let $f: X \rightarrow Y$ be a morphism between two arithmetic varieties over a field. Then there is a pull-back morphism

$$
\widehat{\mathrm{CH}}^{p}(Y, n) \stackrel{f^{*}}{\longrightarrow} \widehat{\mathrm{CH}}^{p}(X, n),
$$

for every $p$ and $n$, compatible with the pull-back maps on the groups $\mathrm{CH}^{p}(X, n)$ and $H_{\mathscr{D}}^{2 p-n}(X, \mathbb{R}(p))$.

- Corollary 4.16 (Homotopy invariance): Let $\pi: X \times \mathbb{A}^{m} \rightarrow X$ be the projection on $X$. Then the pull-back map

$$
\pi^{*}: \widehat{\mathrm{CH}}^{p}(X, n) \rightarrow \widehat{\mathrm{CH}}^{p}\left(X \times \mathbb{A}^{m}, n\right), \quad n \geq 1
$$

is an isomorphism.

- Theorem 5.46 (Product): There exists a product on

$$
\widehat{\mathrm{CH}}^{*}(X, *):=\bigoplus_{p \geq 0, n \geq 0} \widehat{\mathrm{CH}}^{p}(X, n),
$$

which is associative, graded commutative with respect to the degree $n$.

The paper is organized as follows. The first section is a preliminary section. It is devoted to fix the notation and state the main facts used in the rest of the paper. It includes general results on homological algebra, diagrams of complexes, cubical abelian groups and Deligne-Beilinson cohomology. In the second section we recall the definition of the higher Chow groups of Bloch and introduce the complexes of differential forms being the source and target of the regulator map. We proceed in the next section to the definition of the regulator $\rho$ and we prove that it agrees with Beilinson's regulator. In Sections 4 and 5, we develop the theory of higher arithmetic Chow groups. Section 4 is devoted to the definition and basic properties of the higher arithmetic Chow groups and to the comparison with the arithmetic Chow group for $n=0$. Finally, in Section 5 we define the product structure on $\widehat{C H}^{*}(X, *)$ and prove that it is commutative and associative. 
Acknowledgments. This paper originated during a stay of the first author at the CRM (Bellaterra). He is very grateful for the CRM hospitality. During the elaboration of the paper, the second author spent an academic year at the University of Regensburg with a pre-doc grant from the European Network "Arithmetic Algebraic Geometry". She wants to thank all members of the Arithmetic Geometry group, especially U. Jannsen and K. Künneman. We would like to acknowledge M. Levine, H. Gillet and D. Roessler for many useful conversations on the subject of this paper. Finally we would also like to thank the referee of the paper for his or her work.

\section{Preliminaries}

1.1. Notation on (co) chain complexes. We use the standard conventions on (co) chain complexes. By a (co) chain complex we mean a (co) chain complex over the category of abelian groups.

The cochain complex associated to a chain complex $A_{*}$ is simply denoted by $A^{*}$ and the chain complex associated to a cochain complex $A^{*}$ is denoted by $A_{*}$. The translation of a cochain complex $\left(A^{*}, d_{A}\right)$ by an integer $m$ is denoted by $A[m]^{*}$. Recall that $A[m]^{n}=A^{m+n}$ and the differential of $A[m]^{*}$ is $(-1)^{m} d_{A}$. If $\left(A_{*}, d_{A}\right)$ is a chain complex, then the translation of $A_{*}$ by an integer $m$ is denoted by $A[m]_{*}$. In this case the differential is also $(-1)^{m} d_{A}$ but $A[m]_{n}=A_{n-m}$.

The simple complex associated to an iterated chain complex $A_{*}$ is denoted by $s(A)_{*}$ and the analogous notation is used for the simple complex associated to an iterated cochain complex (see [8] $§ 2$ for definitions).

The simple of a cochain map $A^{*} \stackrel{f}{\rightarrow} B^{*}$ is the cochain complex $\left(s(f)^{*}, d_{s}\right)$ with $s(f)^{n}=A^{n} \oplus B^{n-1}$, and differential $d_{s}(a, b)=\left(d_{A} a, f(a)-d_{B} b\right)$. Note that this complex is the cone of $-f$ shifted by 1 . There is an associated long exact sequence

$$
\cdots \rightarrow H^{n}\left(s(f)^{*}\right) \rightarrow H^{n}\left(A^{*}\right) \stackrel{f}{\rightarrow} H^{n}\left(B^{*}\right) \rightarrow H^{n+1}\left(s(f)^{*}\right) \rightarrow \cdots
$$

If $f$ is surjective, there is a quasi-isomorphism

$$
\operatorname{ker} f \stackrel{i}{\rightarrow} s(-f)^{*}, \quad x \mapsto(x, 0),
$$

and if $f$ is injective, there is a quasi-isomorphism

$$
s(f)[1]^{*} \stackrel{\pi}{\rightarrow} B^{*} / A^{*}, \quad(a, b) \mapsto[b] .
$$

Analogously, equivalent results and quasi-isomorphisms can be stated for chain complexes.

Following Deligne [10], given a cochain complex $A^{*}$ and an integer $n$, we denote by $\tau_{\leq n} A^{*}$ the canonical truncation of $A^{*}$ at degree $n$. 
1.2. The simple of a diagram of complexes. We describe here Beilinson's ideas on the simple complexes associated to a diagram of complexes (see [1]). A diagram of chain complexes is a diagram of the form

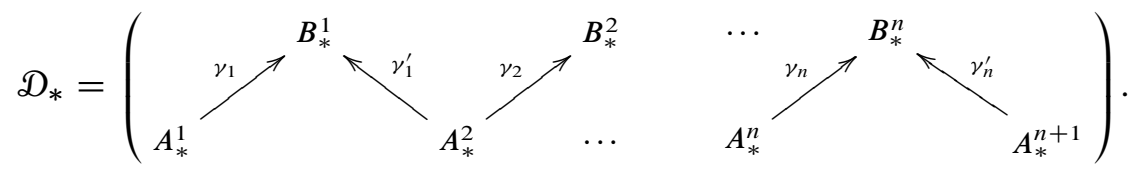

Consider the induced chain morphisms

$$
\begin{array}{lll} 
& \bigoplus_{i=1}^{n+1} A_{*}^{i} \stackrel{\varphi, \varphi_{1}, \varphi_{2}}{\longrightarrow} \bigoplus_{i=1}^{n} B_{*}^{i}, & \\
\varphi_{1}\left(a_{i}\right)=\gamma_{i}\left(a_{i}\right) & & \\
\varphi_{2}\left(a_{i}\right)=\gamma_{i-1}^{\prime}\left(a_{i}\right) & \text { if } a_{i} \in A_{*}^{i}, \\
\varphi\left(a_{i}\right)=\left(\varphi_{1}-\varphi_{2}\right)\left(a_{i}\right)=\left(\gamma_{i}-\gamma_{i-1}^{\prime}\right)\left(a_{i}\right) & \text { if } a_{i} \in A_{*}^{i}, & \text { if } a_{i} \in A_{*}^{i} .
\end{array}
$$

(where we set $\gamma_{n+1}=\gamma_{0}^{\prime}=0$ ). The simple complex associated to the diagram $\mathfrak{D}_{*}$ is defined to be the simple of the morphism $\varphi$ :

$$
s(\mathcal{D})_{*}:=s(\varphi)_{*} .
$$

1.3. Morphisms of diagrams. A morphism between two diagrams $\mathscr{D}_{*}$ and $\mathscr{D}_{*}^{\prime}$ consists of a collection of morphisms

$$
A_{*}^{i} \stackrel{h_{i}^{A}}{\longrightarrow} A_{*}^{\prime i}, \quad B_{*}^{i} \stackrel{h_{i}^{B}}{\longrightarrow} B_{*}^{\prime i},
$$

commuting with the morphisms $\gamma_{i}$ and $\gamma_{i}^{\prime}$, for all $i$. Any morphism of diagrams $\mathscr{D}_{*} \stackrel{h}{\rightarrow} \mathscr{D}_{*}^{\prime}$ induces a morphism on the associated simple complexes $s(\mathscr{D})_{*} \stackrel{s(h)}{\longrightarrow}$ $s\left(\mathscr{D}^{\prime}\right)_{*}$. Observe that if, for every $i, h_{i}^{A}$ and $h_{i}^{B}$ are quasi-isomorphisms, then $s(h)$ is also a quasi-isomorphism.

1.4. Product structure on the simple of a diagram. Let $\mathscr{D}_{*}$ and $\mathscr{D}_{*}^{\prime}$ be two diagrams as 1.4. Consider the diagram obtained by the tensor product of complexes:

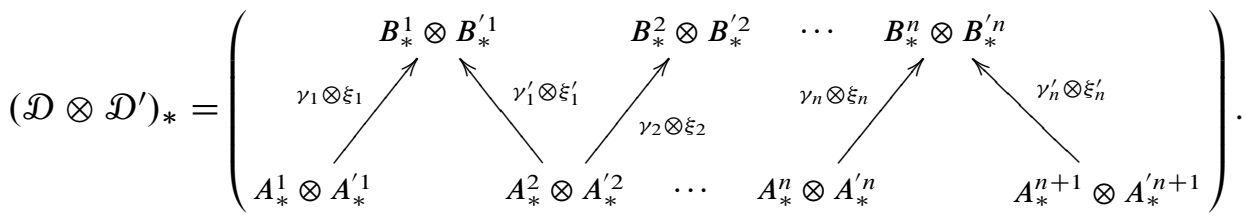


In [1], Beilinson defined, for every $\beta \in \mathbb{Z}$, a morphism

$$
s(\mathscr{D})_{*} \otimes s\left(\mathscr{D}^{\prime}\right)_{*} \stackrel{\star \beta}{\longrightarrow} s\left(\mathscr{D} \otimes \mathscr{D}^{\prime}\right)_{*}
$$

as follows. For $a \in A, a^{\prime} \in A^{\prime}, b \in B$ and $b^{\prime} \in B^{\prime}$, set

$$
\begin{aligned}
& a \star_{\beta} a^{\prime}=a \otimes a^{\prime}, \\
& b \star_{\beta} a^{\prime}=b \otimes\left((1-\beta) \varphi_{1}\left(a^{\prime}\right)+\beta \varphi_{2}\left(a^{\prime}\right)\right), \\
& a \star_{\beta} b^{\prime}=(-1)^{\operatorname{deg} a}\left(\beta \varphi_{1}(a)+(1-\beta) \varphi_{2}(a)\right) \otimes b^{\prime}, \\
& b \star_{\beta} b^{\prime}=0,
\end{aligned}
$$

where the tensor product between elements in different spaces is defined to be zero.

If $B_{*}, C_{*}$ are chain complexes, let

$$
\sigma: s\left(B_{*} \otimes C_{*}\right) \rightarrow s\left(C_{*} \otimes B_{*}\right)
$$

be the map sending $b \otimes c \in B_{n} \otimes C_{m}$ to $(-1)^{n m} c \otimes b \in C_{m} \otimes B_{n}$.

Lemma 1.8 (Beilinson). (i) The map $\star \beta$ is a morphism of complexes.

(ii) For every $\beta, \beta^{\prime} \in \mathbb{Z}, \star \beta$ is homotopic to $\star \beta^{\prime}$.

(iii) There is a commutative diagram

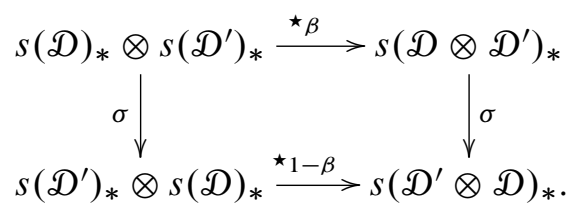

(iv) The products $\star_{0}$ and $\star_{1}$ are associative.

1.5. A specific type of diagrams. In this work we will use diagrams of the following form:

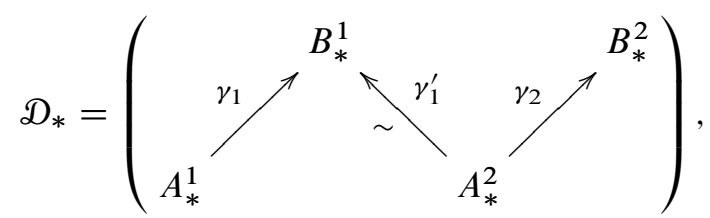

with $\gamma_{1}^{\prime}$ a quasi-isomorphism. For this type of diagrams, since $\gamma_{1}^{\prime}$ is a quasi-isomorphism, we obtain a long exact sequence equivalent to the long exact sequence related to the simple of a morphism. Since a diagram like this induces a map $A_{*}^{1} \longrightarrow B_{*}^{2}$ in the derived category, we obtain

Lemma 1.10. Let $D_{*}$ be a diagram like (1.9). Then there is a well-defined morphism

$$
H_{n}\left(A_{*}^{1}\right) \stackrel{\rho}{\rightarrow} H_{n}\left(B_{*}^{2}\right), \quad\left[a_{1}\right] \mapsto \gamma_{2}\left(\gamma_{1}^{\prime}\right)^{-1} \gamma_{1}\left[a_{1}\right]
$$


Moreover, there is a long exact sequence

$$
\cdots \rightarrow H_{n}\left(s(\mathcal{D})_{*}\right) \rightarrow H_{n}\left(A_{*}^{1}\right) \stackrel{\rho}{\rightarrow} H_{n}\left(B_{*}^{2}\right) \rightarrow H_{n-1}\left(s(\mathscr{D})_{*}\right) \rightarrow \cdots
$$

Consider now a diagram of the form

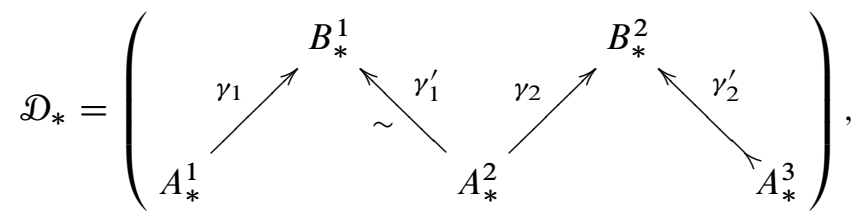

with $\gamma_{1}^{\prime}$ a quasi-isomorphism and $\gamma_{2}^{\prime}$ a monomorphism.

Lemma 1.13. Let $\mathfrak{D}$ be a diagram as (1.12) and let $\mathfrak{D}^{\prime}$ be the diagram

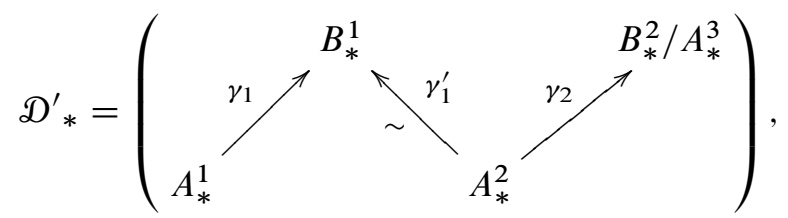

Then there is a quasi-isomorphism between the simple complexes associated to $D$ and to $\mathcal{D}^{\prime}$ :

$$
s(\mathscr{D})_{*} \stackrel{\sim}{\rightarrow} s\left(\mathscr{D}^{\prime}\right)_{*}
$$

Proof. It follows directly from the definition that the simple complex associated to $\mathcal{D}_{*}$ is quasi-isomorphic to the simple associated to the diagram

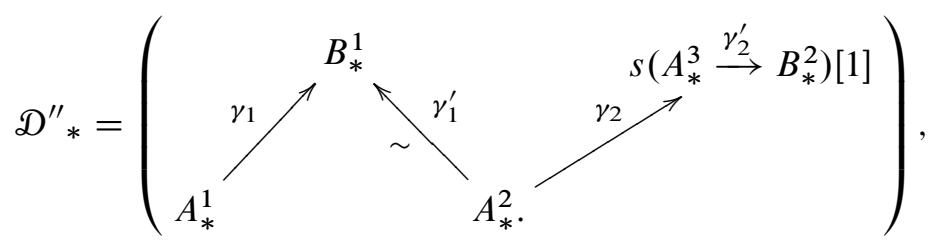

Then the quasi-isomorphism given in (1.3) induces a quasi-isomorphism

$$
s\left(\mathcal{D}^{\prime}\right)_{*} \stackrel{\sim}{\rightarrow} s\left(\mathscr{D}^{\prime \prime}\right)_{*}
$$

as desired.

Corollary 1.16. For any diagram of the form (1.12), there is a long exact sequence

$$
\cdots \rightarrow H_{n}\left(s(\mathcal{D})_{*}\right) \rightarrow H_{n}\left(A_{*}^{1}\right) \stackrel{\rho}{\rightarrow} H_{n-1}\left(s\left(\gamma_{2}^{\prime}\right)\right) \rightarrow H_{n-1}\left(s(\mathcal{D})_{*}\right) \rightarrow \cdots
$$

Proof. It follows from the previous lemma together with Proposition 1.10. 
1.6. Cubical abelian groups and chain complexes. Let $C$. $=\left\{C_{n}\right\}_{n \geq 0}$ be a cubical abelian group with face maps $\delta_{i}^{j}: C_{n} \rightarrow C_{n-1}$, for $i=1, \ldots, n$ and $j=0,1$, and degeneracy maps $\sigma_{i}: C_{n} \rightarrow C_{n+1}$, for $i=1, \ldots, n+1$. Let $D_{n} \subset C_{n}$ be the subgroup of degenerate elements of $C_{n}$, and let $\widetilde{C}_{n}=C_{n} / D_{n}$.

Let $C_{*}$ denote the associated chain complex, that is, the chain complex whose $n$-th graded piece is $C_{n}$ and whose differential is given by $\delta=\sum_{i=1}^{n} \sum_{j=0,1}(-1)^{i+j} \delta_{i}^{j}$. Thus $D_{*}$ is a subcomplex and $\widetilde{C}_{*}$ is a quotient complex. We fix the normalized chain complex associated to $C$., $N C_{*}$, to be the chain complex whose $n$-th graded group is $N C_{n}:=\bigcap_{i=1}^{n} \operatorname{ker} \delta_{i}^{1}$, and whose differential is $\delta=\sum_{i=1}^{n}(-1)^{i} \delta_{i}^{0}$. It is wellknown that there is a decomposition of chain complexes $C_{*} \cong N C_{*} \oplus D_{*}$ giving an isomorphism $N C_{*} \cong \widetilde{C}_{*}$.

For certain cubical abelian groups, the normalized chain complex can be further simplified, up to homotopy equivalence, by considering the elements which belong to the kernel of all faces but $\delta_{1}^{0}$.

Definition 1.18. Let $C$. be a cubical abelian group. Let $N_{0} C_{*}$ be the complex defined by

$$
N_{0} C_{n}=\bigcap_{i=1}^{n} \operatorname{ker} \delta_{i}^{1} \cap \bigcap_{i=2}^{n} \operatorname{ker} \delta_{i}^{0}, \quad \text { and differential } \delta=-\delta_{1}^{0} .
$$

The proof of the next proposition is analogous to the proof of Theorem 4.4.2 in [2]. The result is proved there only for the cubical abelian group defining the higher Chow complex (see $\S 2.1$ below). We give here the abstract version of the statement, valid for a certain type of cubical abelian groups.

Proposition 1.20. Let $C$. be a cubical abelian group. Assume that it comes equipped with a collection of maps

$$
h_{j}: C_{n} \rightarrow C_{n+1}, \quad j=1, \ldots, n,
$$

such that, for any $l=0,1$, the following identities are satisfied:

$$
\begin{aligned}
& \delta_{j}^{1} h_{j}=\delta_{j+1}^{1} h_{j}=s_{j} \delta_{j}^{1}, \\
& \delta_{j}^{0} h_{j}=\delta_{j+1}^{0} h_{j}=\mathrm{id}, \\
& \delta_{i}^{l} h_{j}= \begin{cases}h_{j-1} \delta_{i}^{l}, & i<j, \\
h_{j} \delta_{i-1}^{l}, & i>j+1 .\end{cases}
\end{aligned}
$$

Then the inclusion of complexes

$$
i: N_{0} C_{*} \hookrightarrow N C_{*}
$$

is a homotopy equivalence. 
Proof. Let $g_{j}: N C_{n} \rightarrow N C_{n+1}$ be defined as $g_{j}=(-1)^{n-j} h_{n-j}$ if $0 \leq j \leq n-1$ and $g_{j}=0$ otherwise. Then there is a well-defined morphism of chain complexes

$$
H_{j}=\left(\mathrm{Id}+\delta g_{j}+g_{j} \delta\right): N C_{*} \rightarrow N C_{*} .
$$

This morphism is homotopically equivalent to the identity.

Let $x \in N C_{n}$ and $0 \leq j \leq n-1$. Then

$$
\begin{aligned}
\delta h_{n-j}(x) & =\sum_{i=1}^{n+1}(-1)^{i} \delta_{i}^{0} h_{n-j}(x) \\
& =\sum_{i=1}^{n-j-1}(-1)^{i} h_{n-j-1} \delta_{i}^{0}(x)+\sum_{i=n-j+2}^{n+1}(-1)^{i} h_{n-j} \delta_{i-1}^{0}(x), \\
h_{n-j-1} \delta(x) & =\sum_{i=1}^{n}(-1)^{i} h_{n-j-1} \delta_{i}^{0}(x) .
\end{aligned}
$$

Hence,

$$
\begin{aligned}
& \delta g_{j}(x)+g_{j} \delta(x) \\
& =(-1)^{n-j} \sum_{i=n-j+1}^{n}(-1)^{i-1} h_{n-j} \delta_{i}^{0}(x)+(-1)^{n-j-1} \sum_{i=n-j}^{n}(-1)^{i} h_{n-j-1} \delta_{i}^{0}(x) .
\end{aligned}
$$

We consider the decreasing filtration $G^{*}$ of $N C_{*}$, given by

$$
G^{j} N C_{n}=\left\{x \in N C_{n} \mid \delta_{i}^{0}(x)=0, i>\max (n-j, 1)\right\} .
$$

Then $G^{0} N C_{*}=N C_{*}$ and for $j \geq n-1, G^{j} N C_{n}=N_{0} C_{n}$. If $x \in G^{j+1} N C_{*}$, then $\delta g_{j}(x)+g_{j} \delta(x)=0$ and thus, $H_{j}(x)=x$. Moreover, if $x \in G^{j} N C_{*}$, then $H_{j}(x) \in G^{j+1} N C_{*}$. Thus, $H_{j}$ is the projector from $G^{j} N C_{*}$ to $G^{j+1} N C_{*}$.

Thus, the morphism $\varphi: N C_{*} \rightarrow N_{0} C_{*}$ given, on $N C_{n}$, by $\varphi:=H_{n-2} \circ \cdots \circ H_{0}$ forms a chain morphism homotopically equivalent to the identity. Moreover $\varphi$ is the projector from $N C *$ to $N_{0} C_{*}$. Hence, $\varphi \circ i$ is the identity of $N_{0} C_{*}$ while $i \circ \varphi$ is homotopically equivalent to the identity of $N C_{*}$.

Remark 1.23. To every cubical abelian group $C$. there are associated four chain complexes: $C_{*}, N C_{*}, N_{0} C_{*}$ and $\widetilde{C}_{*}$. In some situations it will be necessary to consider the cochain complexes associated to these chain complexes. In this case we will write, respectively, $C^{*}, N C^{*}, N_{0} C^{*}$ and $\widetilde{C}^{*}$.

1.7. Cubical cochain complexes. Let $X^{*}$ be a cubical cochain complex. Then, for every $m$, the cochain complexes $N X_{m}^{*}, N_{0} X_{m}^{*}$ and $\widetilde{X}_{m}^{*}$ are defined. 
Proposition 1.24. Let $X^{*}, Y^{*}$ be two cubical cochain complexes and let $f: X^{*} \rightarrow$ $Y^{*}$ be a morphism. Assume that for every $m$, the cochain morphism

$$
X_{m}^{*} \stackrel{f_{m}}{\longrightarrow} Y_{m}^{*}
$$

is a quasi-isomorphism. Then the induced morphisms

$$
N X_{m}^{*} \stackrel{f_{m}}{\longrightarrow} N Y_{m}^{*} \text { and } \tilde{C}_{m}^{*} \stackrel{f_{m}}{\longrightarrow} \tilde{Y}_{m}^{*}
$$

are quasi-isomorphisms.

Proof. The proposition follows from the decompositions

$H^{r}\left(X_{m}^{*}\right)=H^{r}\left(N X_{m}^{*}\right) \oplus H^{r}\left(D X_{m}^{*}\right) \quad$ and $\quad H^{r}\left(Y_{m}^{*}\right)=H^{r}\left(N Y_{m}^{*}\right) \oplus H^{r}\left(D Y_{m}^{*}\right)$,

and the fact that $f_{m}$ induces cochain maps

$$
N X_{m}^{*} \stackrel{f_{m}}{\longrightarrow} N Y_{m}^{*} \text { and } D X_{m}^{*} \stackrel{f_{m}}{\longrightarrow} D Y_{m}^{*}
$$

Proposition 1.25. Let $X^{*}$ be a cubical cochain complex. Then the natural morphism

$$
H^{r}\left(N X_{n}^{*}\right) \stackrel{f}{\rightarrow} N H^{r}\left(X_{n}^{*}\right)
$$

is an isomorphism for all $n \geq 0$.

Proof. The cohomology groups $H^{r}\left(X^{*}\right)$ have a cubical abelian group structure. Hence there is a decomposition

$$
H^{r}\left(X_{.}^{*}\right)=N H^{r}\left(X_{*}^{*}\right) \oplus D H^{r}\left(X_{*}^{*}\right) .
$$

In addition, there is a decomposition $X_{n}^{*}=N X_{n}^{*} \oplus D X_{n}^{*}$. Therefore

$$
H^{r}\left(X_{.}^{*}\right)=H^{r}\left(N X_{.}^{*}\right) \oplus H^{r}\left(D X_{.}^{*}\right) .
$$

The lemma follows from the fact that the identity morphism in $H^{r}\left(X^{*}\right)$ maps $N H^{r}\left(X_{.}^{*}\right)$ to $H^{r}\left(N X_{.}^{*}\right)$ and $D H^{r}\left(X_{.}^{*}\right)$ to $H^{r}\left(D X_{.}^{*}\right)$.

1.8. Deligne-Beilinson cohomology. In this paper we use the definitions and conventions on Deligne-Beilinson cohomology given in [5] and [8], chapter 5.

One denotes $\mathbb{R}(p)=(2 \pi i)^{p} \cdot \mathbb{R} \subset \mathbb{C}$. Let $X$ be a complex algebraic manifold and denote by $E_{\log , \mathbb{R}}^{*}(X)(p)$ the complex of real differential forms with logarithmic singularities along infinity, twisted by $p$. Let $\left(\mathscr{D}_{\log }^{*}(X, p), d_{\mathscr{D}}\right)$ be the Deligne complex of differential forms with logarithmic singularities, as described in [5]. It computes real Deligne-Beilinson cohomology of $X$, that is,

$$
H^{n}\left(D_{\log }^{*}(X, p)\right)=H_{\mathscr{D}}^{n}(X, \mathbb{R}(p)) .
$$


This complex is functorial on $X$.

The product structure in Deligne-Beilinson cohomology can be described by a cochain morphism on the Deligne complex (see [5]):

$$
\begin{aligned}
\mathscr{D}_{\log }^{n}(X, p) \otimes \mathscr{D}_{\log }^{m}(X, q) & \stackrel{\longrightarrow}{\longrightarrow} \mathscr{D}_{\log }^{n+m}(X, p+q), \\
x \otimes y & \longmapsto x \bullet y .
\end{aligned}
$$

This product satisfies the expected relations:

(1) Graded commutativity: $\quad x \bullet y=(-1)^{n m} y \bullet x$.

(2) Leibniz rule: $\quad d_{\mathscr{D}}(x \bullet y)=d_{\mathscr{D}} x \bullet y+(-1)^{n} x \bullet d_{\mathscr{D}} y$.

Proposition 1.26. The Deligne product $\bullet$ is associative up to a natural homotopy, i.e. there exists

$$
h: \mathscr{D}_{\log }^{r}(X, p) \otimes \mathscr{D}_{\log }^{s}(X, q) \otimes \mathscr{D}_{\log }^{t}(X, l) \rightarrow \mathscr{D}_{\log }^{r+s+t}(X, p+q+l)
$$

such that

$d_{\mathscr{D}} h\left(\omega_{1} \otimes \omega_{2} \otimes \omega_{3}\right)+h d_{\mathscr{D}}\left(\omega_{1} \otimes \omega_{2} \otimes \omega_{3}\right)=\left(\omega_{1} \bullet \omega_{2}\right) \bullet \omega_{3}-\omega_{1} \bullet\left(\omega_{2} \bullet \omega_{3}\right)$.

Furthermore, if $\omega_{1} \in \mathscr{D}_{\log }^{2 p}(X, p), \omega_{2} \in \mathscr{D}_{\log }^{2 q}(X, q)$ and $\omega_{3} \in \mathscr{D}_{\log }^{2 l}(X, l)$ satisfy $d_{\mathscr{D}} \omega_{i}=0$ for all $i$, then

$$
h\left(\omega_{1} \otimes \omega_{2} \otimes \omega_{3}\right)=0 .
$$

Proof. This is [5], Theorem 3.3.

1.9. Cohomology with supports. Let $Z$ be a closed subvariety of a complex algebraic manifold $X$. Consider the complex $\mathscr{D}_{\log }^{*}(X \backslash Z, p)$, i.e. the Deligne complex of differential forms in $X \backslash Z$ with logarithmic singularities along $Z$ and infinity.

Definition 1.28. The Deligne complex with supports in $Z$ is defined to be

$$
\mathscr{D}_{\log , Z}^{*}(X, p)=s\left(\mathscr{D}_{\log }^{*}(X, p) \rightarrow \mathscr{D}_{\log }^{*}(X \backslash Z, p)\right) .
$$

The Deligne-Beilinson cohomology with supports in $Z$ is defined as the cohomology groups of the Deligne complex with supports in $Z$ :

$$
H_{\mathscr{D}, Z}^{n}(X, \mathbb{R}(p)):=H^{n}\left(\mathscr{D}_{\log , Z}^{*}(X, p)\right) .
$$

Lemma 1.29. Let $Z, W$ be two closed subvarieties of a complex algebraic manifold $X$. Then there is a short exact sequence of Deligne complexes,

$$
\begin{aligned}
0 \rightarrow \mathscr{D}_{\log }^{*}(X \backslash Z \cap W, p) \stackrel{i}{\longrightarrow} & \mathscr{D}_{\log }^{*}(X \backslash Z, p) \oplus \mathscr{D}_{\log }^{*}(X \backslash W, p) \\
& \stackrel{j}{\longrightarrow} \mathscr{D}_{\log }^{*}(X \backslash Z \cup W, p) \rightarrow 0,
\end{aligned}
$$

where $i(\alpha)=(\alpha, \alpha)$ and $j(\alpha, \beta)=-\alpha+\beta$. 
Proof. It follows from [7], Theorem 3.6.

In addition, Deligne-Beilinson cohomology with supports satisfies a semipurity property. Namely, let $Z$ be a codimension $p$ subvariety of an equidimensional complex manifold $X$, and let $Z_{1}, \ldots, Z_{r}$ be its codimension $p$ irreducible components. Then

$$
H_{\mathscr{D}, Z}^{n}(X, \mathbb{R}(p))= \begin{cases}0, & n<2 p, \\ \bigoplus_{i=1}^{r} \mathbb{R}\left[Z_{i}\right], & n=2 p .\end{cases}
$$

For the next proposition, let $\delta_{Z}$ denote the current integration along an irreducible variety $Z$. In the sequel we will use the conventions of [8] $\$ 5.4$ with respect to the current associated to a locally integrable form and to the current $\delta_{Z}$.

Proposition 1.31. Let $X$ be an equidimensional complex algebraic manifold and $Z$ a codimension $p$ irreducible subvariety of $X$. Let $j: X \rightarrow \bar{X}$ be a smooth compactification of $X$ (with a normal crossing divisor as its complement) and $\bar{Z}$ the closure of $Z$ in $\bar{X}$. The isomorphism

$$
\mathrm{cl}: \mathbb{R}[Z] \stackrel{\cong}{\rightarrow} H_{\mathscr{D}, Z}^{2 p}(X, \mathbb{R}(p))
$$

sends $[Z]$ to $\left[\left(j^{*} w, j^{*} g\right)\right]$, for any $[(w, g)] \in H_{\mathscr{D}, \bar{Z}}^{2 p}(\bar{X}, \mathbb{R}(p))$ satisfying the relation of currents in $\bar{X}$

$$
-2 \partial \bar{\partial}[g]=[w]-\delta_{\bar{Z}}
$$

Proof. See [8], Proposition 5.58.

In particular, assume that $Z=\operatorname{div}(f)$ is a principal divisor, where $f$ is a rational function on $X$. Then $[Z]$ is represented by the couple

$$
\left(0,-\frac{1}{2} \log (f \bar{f})\right) \in H_{\mathscr{D}, Z}^{2 p}(X, \mathbb{R}(p)) .
$$

The definition of the cohomology with support in a subvariety can be extended to the definition of the cohomology with support in a set of subvarieties of $X$. We explain here the case used in the sequel. Let $\mathcal{Z}^{p}$ be a subset of the set of codimension $p$ closed subvarieties of $X$, that is closed under finite unions. The inclusion of subsets turns $\mathcal{Z}^{p}$ into a directed ordered set. We define the complex

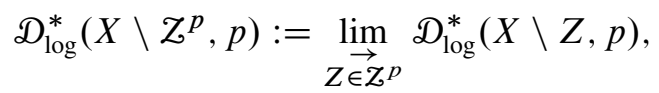

which is provided with an injective map

$$
\mathcal{D}_{\log }^{*}(X, p) \stackrel{i}{\rightarrow} \mathscr{D}_{\log }^{*}\left(X \backslash Z^{p}, p\right) .
$$


As above, we define

$$
\mathcal{D}_{\log , Z^{p}}^{*}(X, p):=s(i)^{*}
$$

and the Deligne-Beilinson cohomology with supports in $Z^{p}$ as

$$
H_{\mathscr{D}, Z^{p}}^{n}(X, \mathbb{R}(p)):=H^{n}\left(\mathcal{D}_{\log , Z^{p}}^{*}(X, p)\right) .
$$

1.10. Real varieties. A real variety $X$ consists of a couple $\left(X_{\mathbb{C}}, F_{\infty}\right)$, with $X_{\mathbb{C}}$ a complex algebraic manifold and $F_{\infty}$ an antilinear involution of $X_{\mathbb{C}}$.

If $X=\left(X_{\mathbb{C}}, F_{\infty}\right)$ is a real variety, we will denote by $\sigma$ the involution of $\mathscr{D}_{\log }^{n}\left(X_{\mathbb{C}}, p\right)$ given by

$$
\sigma(\eta)=\overline{F_{\infty}^{*} \eta}
$$

Then the real Deligne-Beilinson cohomology of $X$ is defined by

$$
H_{\mathscr{D}}^{n}(X, \mathbb{R}(p)):=H_{\mathscr{D}}^{n}\left(X_{\mathbb{C}}, \mathbb{R}(p)\right)^{\sigma},
$$

where the superscript $\sigma$ means the fixed part under $\sigma$.

The real cohomology of $X$ is expressed as the cohomology of the real Deligne complex

$$
\mathscr{D}_{\log }^{n}(X, p):=\mathscr{D}_{\log }^{n}\left(X_{\mathbb{C}}, p\right)^{\sigma},
$$

i.e. there is an isomorphism

$$
H_{\mathscr{D}}^{n}(X, \mathbb{R}(p)) \cong H^{n}\left(\mathcal{D}_{\log }^{n}(X, p), d_{\mathscr{D}}\right) .
$$

1.11. Truncated Deligne complex. In the rest of the work, we will consider the Deligne complex (canonically) truncated at degree $2 p$. For simplicity we will denote it by

$$
\tau \mathcal{D}_{\log }^{*}(X, p)=\tau_{\leq 2 p} \mathcal{D}_{\log }^{*}(X, p) .
$$

The truncated Deligne complex with supports in a variety $Z$ is denoted by $\tau \mathscr{D}_{\log , Z}^{*}(X, p)=\tau_{\leq 2 p} \mathscr{D}_{\log , Z}^{*}(X, p)$ and the truncated Deligne complex with supports in $\mathcal{Z}^{p}$ is denoted by $\tau \mathscr{D}_{\log , Z^{p}}^{*}(X, p)=\tau_{\leq 2 p} \mathscr{D}_{\log , Z^{p}}^{*}(X, p)$.

Note that, since the truncation is not an exact functor, it is not true that $\tau \mathscr{D}_{\log , Z^{p}}^{*}(X, p)$ is the simple complex of the map $\tau \mathscr{D}_{\log }^{*}(X, p) \rightarrow \tau \mathscr{D}_{\log }^{*}\left(X \backslash Z^{p}, p\right)$.

\section{Differential forms and higher Chow groups}

In this section we construct a complex of differential forms which is quasi-isomorphic to the complex $Z^{p}(X, *)_{0} \otimes \mathbb{R}$. This last complex computes the higher algebraic Chow groups introduced by Bloch in [3] with real coefficients. The key point of this construction is the set of isomorphisms given in (1.30). 
This complex is very similar to the complex introduced by Bloch in [4] in order to construct the cycle map for the higher Chow groups. In both constructions one considers a 2-iterated complex of differential forms on a cubical or simplicial scheme. Since this leads to a second quadrant spectral sequence, to avoid convergence problems, one has to truncate the complexes involved. The main difference between both constructions is the direction of the truncation. We truncate the 2 -iterated complex at the degree given by the differential forms, while in loc. cit. the complex is truncated at the degree given by the simplicial scheme.

2.1. The cubical Bloch complex. We recall here the definition and main properties of the higher Chow groups defined by Bloch in [3]. Initially, they were defined using the chain complex associated to a simplicial abelian group. However, since we are interested in the product structure, it is more convenient to use the cubical presentation, as given by Levine in [19].

Fix a base field $k$ and let $\mathbb{P}^{1}$ be the projective line over $k$. Let $\square=\mathbb{P}^{1} \backslash\{1\}\left(\cong \mathbb{A}^{1}\right)$. The cartesian product $\left(\mathbb{P}^{1}\right)^{\cdot}$ has a cocubical scheme structure. For $i=1, \ldots, n$, we denote by $t_{i} \in(k \cup\{\infty\}) \backslash\{1\}$ the absolute coordinate of the $i$-th factor. Then the coface and codegeneracy maps are defined as

$$
\begin{aligned}
& \delta_{0}^{i}\left(t_{1}, \ldots, t_{n}\right)=\left(t_{1}, \ldots, t_{i-1}, 0, t_{i}, \ldots, t_{n}\right), \\
& \delta_{1}^{i}\left(t_{1}, \ldots, t_{n}\right)=\left(t_{1}, \ldots, t_{i-1}, \infty, t_{i}, \ldots, t_{n}\right), \\
& \sigma^{i}\left(t_{1}, \ldots, t_{n}\right)=\left(t_{1}, \ldots, t_{i-1}, t_{i+1}, \ldots, t_{n}\right) .
\end{aligned}
$$

Then $\square$ inherits a cocubical scheme structure from that of $\left(\mathbb{P}^{1}\right)^{\prime}$. An $r$-dimensional face of $\square^{n}$ is any subscheme of the form $\delta_{j_{1}}^{i_{1}} \cdots \delta_{j_{r}}^{i_{r}}\left(\square^{n-r}\right)$.

We have chosen to represent $\mathbb{A}^{1}$ as $\mathbb{P}^{1} \backslash\{1\}$ so that the face maps are represented by the inclusion at zero and the inclusion at infinity. In this way the cubical structure of $\square$ is compatible with the cubical structure of $\left(\mathbb{P}^{1}\right)^{\cdot}$ in [9]. In the literature the usual representation $\mathbb{A}^{1}=\mathbb{P}^{1} \backslash\{\infty\}$ is often used. We will translate from one definition to the other by using the involution

$$
x \longmapsto \frac{x}{x-1} .
$$

This involution has the fixed points $\{0,2\}$ and interchanges the points 1 and $\infty$.

Let $X$ be an equidimensional quasi-projective variety of dimension $d$ over the field $k$. Let $Z^{p}(X, n)$ be the free abelian group generated by the codimension $p$ closed irreducible subvarieties of $X \times \square^{n}$, which intersect properly all the faces of $\square^{n}$. The pull-back by the coface and codegeneracy maps of $\square^{\cdot}$ endow $Z^{p}(X, \cdot)$ with a cubical abelian group structure. Let $\left(Z^{p}(X, *), \delta\right)$ be the associated chain complex (see §1.6) and consider the normalized chain complex associated to $Z^{p}(X, *)$,

$$
Z^{p}(X, n)_{0}:=N Z^{p}(X, n)=\bigcap_{i=1}^{n} \operatorname{ker} \delta_{i}^{1} .
$$


Definition 2.2. Let $X$ be a quasi-projective equidimensional variety over a field $k$. The higher Chow groups defined by Bloch are

$$
\mathrm{CH}^{p}(X, n):=H_{n}\left(Z^{p}(X, *)_{0}\right) .
$$

Let $N_{0}$ be the refined normalized complex of Definition (1.18). Let $Z^{p}(X, *)_{00}$ be the complex with

$$
Z^{p}(X, n)_{00}:=N_{0} Z^{p}(X, n)=\bigcap_{i=1}^{n} \operatorname{ker} \delta_{i}^{1} \cap \bigcap_{i=2}^{n} \operatorname{ker} \delta_{i}^{0} .
$$

Fix $n \geq 0$. For every $j=1, \ldots, n$, we define a map

$$
\begin{aligned}
\square^{n+1} & \stackrel{h^{j}}{\longrightarrow} \square^{n}, \\
\left(t_{1}, \ldots, t_{n+1}\right) & \longmapsto\left(t_{1}, \ldots, t_{j-1}, 1-\left(t_{j}-1\right)\left(t_{j+1}-1\right), t_{j+2}, \ldots, t_{n+1}\right) .
\end{aligned}
$$

The refined normalized complex of [2] $\$ 4.4$ is given by considering the elements in the kernel of all faces but $\delta_{1}^{1}$, instead of $\delta_{1}^{0}$ like here. Taking this into account, together with the involution (2.1), the map $h^{j}$ agrees with the map denoted by $h^{n-j}$ in [2] $\S 4.4$. Therefore, the maps $h_{j}$ are smooth, hence flat, so they induce pull-back maps

$$
h_{j}: Z^{p}(X, n) \longrightarrow Z^{p}(X, n+1), \quad j=1, \ldots, n+1,
$$

that satisfy the conditions of Proposition 1.20. Therefore the inclusion

$$
Z^{p}(X, n)_{00}:=N_{0} Z^{p}(X, n) \rightarrow Z^{p}(X, n)_{0}
$$

is a homotopy equivalence (see [2] §4.4).

2.2. Functoriality. It follows easily from the definition that the complex $Z^{p}(X, *)_{0}$ is covariant with respect to proper maps (with a shift in the grading) and contravariant for flat maps.

Let $f: X \rightarrow Y$ be an arbitrary map between two smooth varieties $X, Y$. Let $Z_{f}^{p}(Y, n)_{0} \subset Z^{p}(Y, n)_{0}$ be the subgroup generated by the codimension $p$ irreducible subvarieties $Z \subset Y \times \square^{n}$, intersecting properly the faces of $\square^{n}$ and such that the pullback $X \times Z$ intersects properly the graph of $f, \Gamma_{f}$. Then $Z_{f}^{p}(Y, *)_{0}$ is a chain complex and the inclusion of complexes $Z_{f}^{p}(Y, *)_{0} \subseteq Z^{p}(Y, *)_{0}$ is a quasi-isomorphism. Moreover, the pull-back by $f$ is defined for algebraic cycles in $Z_{f}^{p}(Y, *)_{0}$ and hence there is a well-defined pull-back morphism

$$
\mathrm{CH}^{p}(Y, n) \stackrel{f^{*}}{\longrightarrow} \mathrm{CH}^{p}(X, n) .
$$

A proof of this fact can be found in [20], §3.5. See also [18]. 
2.3. Product structure. Let $X$ and $Y$ be quasi-projective smooth varieties over $k$. Then there is a chain morphism

$$
s\left(Z^{p}(X, *)_{0} \otimes Z^{q}(Y, *)_{0}\right) \stackrel{\cup}{\rightarrow} Z^{p+q}(X \times Y, *)_{0}
$$

inducing exterior products

$$
\mathrm{CH}^{p}(X, n) \otimes \mathrm{CH}^{q}(Y, m) \stackrel{\cup}{\rightarrow} \mathrm{CH}^{p+q}(X \times Y, n+m) .
$$

More concretely, let $Z$ be a codimension $p$ irreducible subvariety of $X \times \square^{n}$, intersecting properly the faces of $\square^{n}$ and let $W$ be a codimension $q$ irreducible subvariety of $Y \times \square^{m}$, intersecting properly the faces of $\square^{m}$. Then the codimension $p+q$ subvariety

$$
Z \times W \subseteq X \times \square^{n} \times Y \times \square^{m} \cong X \times Y \times \square^{n} \times \square^{m} \cong X \times Y \times \square^{n+m},
$$

intersects properly the faces of $\square^{n+m}$. By linearity, we obtain a morphism

$$
Z^{p}(X, n) \otimes Z^{q}(Y, m) \stackrel{\cup}{\rightarrow} Z^{p+q}(X \times Y, n+m) .
$$

It induces a chain morphism on the normalized complexes

$$
s\left(Z^{p}(X, *)_{0} \otimes Z^{q}(Y, *)_{0}\right) \stackrel{\cup}{\rightarrow} Z^{p+q}(X \times Y, *)_{0},
$$

and hence there is an external product

$$
\cup: \mathrm{CH}^{p}(X, n) \otimes \mathrm{CH}^{q}(Y, m) \rightarrow \mathrm{CH}^{p+q}(X \times Y, n+m),
$$

for all $p, q, n, m$.

If $X$ is smooth, then the pull-back by the diagonal map $\Delta: X \rightarrow X \times X$ is defined on the higher Chow groups, $\mathrm{CH}^{p}(X \times X, *) \stackrel{\Delta^{*}}{\longrightarrow} \mathrm{CH}^{p}(X, *)$. Therefore, for all $p$, $q, n, m$, we obtain an internal product

$$
\cup: \mathrm{CH}^{p}(X, n) \otimes \mathrm{CH}^{q}(X, m) \rightarrow \mathrm{CH}^{p+q}(X \times X, n+m) \stackrel{\Delta^{*}}{\longrightarrow} \mathrm{CH}^{p+q}(X, n+m) .
$$

In the derived category of chain complexes, the internal product is given by the morphism

$$
\begin{aligned}
s\left(Z^{p}(X, *)_{0} \otimes Z^{q}(X, *)_{0}\right) \stackrel{U}{\longrightarrow} & Z_{\uparrow}^{p+q}(X \times X, *)_{0} \\
& Z_{\Delta}^{p+q}(X \times X, *)_{0} \stackrel{\Delta^{*}}{\longrightarrow} Z^{p+q}(X, *)_{0} .
\end{aligned}
$$

Proposition 2.7. Let $X$ be a quasi-projective smooth variety over $k$. The pairing (2.6) defines an associative product on $\mathrm{CH}^{*}(X, *)=\bigoplus_{p, n} \mathrm{CH}^{p}(X, n)$. This product is graded commutative with respect to the degree given by $n$.

Proof. See [19], Theorem 5.2. 
2.4. Differential forms and affine lines. For every $n, p \geq 0$, let $\tau \mathcal{D}_{\log }^{*}\left(X \times \square^{n}, p\right)$ be the truncated Deligne complex of differential forms in $X \times \square^{n}$, with logarithmic singularities at infinity. The structural maps of the cocubical scheme $\square$ ' induce a cubical structure on $\tau \mathscr{D}_{\log }^{r}\left(X \times \square^{*}, p\right)$ for every $r$ and $p$.

Consider the 2-iterated cochain complex

$$
\mathcal{D}_{\mathbb{A}}^{r,-n}(X, p)=\tau \mathcal{D}_{\log }^{r}\left(X \times \square^{n}, p\right),
$$

with differential $\left(d_{\mathscr{D}}, \delta=\sum_{i=1}^{n}(-1)^{i}\left(\delta_{i}^{0}-\delta_{i}^{1}\right)\right)$. Let

$$
\mathscr{D}_{\mathbb{A}}^{*}(X, p)=s\left(\mathscr{D}_{\mathbb{A}}^{*, *}(X, p)\right)
$$

be the associated simple complex. Hence its differential $d_{s}$ in $\mathscr{D}_{\mathbb{A}}^{*}(X, p)$ is given, for every $\alpha \in \mathcal{D}_{\mathbb{A}}^{r,-n}(X, p)$, by $d_{S}(\alpha)=d_{\mathscr{D}}(\alpha)+(-1)^{r} \delta(\alpha)$. Since we are using cubical structures, this complex does not compute the right cohomology and we have to normalize it.

For every $r, n$ we write

$$
\mathscr{D}_{\mathbb{A}}^{r,-n}(X, p)_{0}=\tau \mathcal{D}_{\log }^{r}\left(X \times \square^{n}, p\right)_{0}:=N \tau \mathscr{D}_{\log }^{r}\left(X \times \square^{n}, p\right) .
$$

Therefore $\mathcal{D}_{\mathbb{A}}^{*, *}(X, p)_{0}$ is the normalized 2-iterated complex and we denote by $D_{\mathbb{A}}^{*}(X, p)_{0}$ the associated simple complex.

Proposition 2.8. The natural morphism of complexes

$$
\tau \mathscr{D}_{\log }^{*}(X, p)=\mathscr{D}_{\mathbb{A}}^{*, 0}(X, p)_{0} \rightarrow \mathscr{D}_{\mathbb{A}}^{*}(X, p)_{0}
$$

is a quasi-isomorphism.

Proof. Consider the second quadrant spectral sequence with $E_{1}$ term given by

$$
E_{1}^{r,-n}=H^{r}\left(\mathscr{D}_{\mathbb{A}}^{*,-n}(X, p)_{0}\right) .
$$

Since

$$
\mathscr{D}_{\mathbb{A}}^{r,-n}(X, p)_{0}=0, \quad \text { for } r<0 \text { or } r>2 p,
$$

this spectral sequence converges to the cohomology groups $H^{*}\left(D_{\mathbb{A}}^{*}(X, p)_{0}\right)$. This is the main reason why we use the truncated complexes.

If we see that, for all $n>0$, the cohomology of the complex $\mathcal{D}_{\mathbb{A}}^{*,-n}(X, p)_{0}$ is zero, the spectral sequence degenerates and the proposition is proved. By the homotopy invariance of Deligne-Beilinson cohomology, there is an isomorphism

$$
\delta_{1}^{1} \circ \cdots \circ \delta_{1}^{1}: H^{*}\left(\tau \mathcal{D}_{\log }^{*}\left(X \times \square^{n}, p\right)\right) \rightarrow H^{*}\left(\tau \mathcal{D}_{\log }^{*}(X, p)\right) .
$$

By definition, the image of $H^{*}\left(\tau \mathscr{D}_{\log }^{*}\left(X \times \square^{n}, p\right)_{0}\right)$ by this isomorphism is zero. Since $H^{*}\left(\tau \mathscr{D}_{\log }^{*}\left(X \times \square^{n}, p\right)_{0}\right)$ is a direct summand of $H^{*}\left(\tau \mathscr{D}_{\log }^{*}\left(X \times \square^{n}, p\right)\right)$, it vanishes for all $n>0$. 
We define the complex $\mathcal{D}_{\mathbb{A}}^{*}(X, p)_{00}$ to be the simple complex associated to the 2-iterated complex with

$$
\mathcal{D}_{\mathbb{A}}^{r,-n}(X, p)_{00}=N_{0} \tau \mathcal{D}_{\log }^{r}\left(X \times \square^{n}, p\right) .
$$

Corollary 2.9. The natural morphism of complexes

$$
\tau \mathcal{D}_{\log }^{*}(X, p)=\mathscr{D}_{\mathbb{A}}^{*, 0}(X, p)_{00} \rightarrow \mathscr{D}_{\mathbb{A}}^{*}(X, p)_{00}
$$

is a quasi-isomorphism.

Proof. It follows from Proposition 2.8, Proposition 1.20 (using as maps $\left\{h_{j}\right\}$ the ones induced by the maps $h^{j}$ defined in 2.3) and Proposition 1.24.

2.5. A complex with differential forms for the higher Chow groups. Let $Z_{n, X}^{p}$ be the set of all codimension $p$ closed subvarieties of $X \times \square^{n}$ intersecting properly the faces of $\square^{n}$. We consider it as a set ordered by the inclusion relation. When there is no source of confusion, we simply write $\mathcal{Z}_{n}^{p}$ or even $\mathcal{Z}^{p}$. Consider the cubical abelian group

$$
\mathscr{H}^{p}(X, *):=H_{\mathscr{D}, \mathcal{Z}_{*}^{p}}^{2 p}\left(X \times \square^{*}, \mathbb{R}(p)\right),
$$

with faces and degeneracies induced by those of $\square$. Let $\mathscr{H}^{p}(X, *)_{0}$ be the associated normalized complex.

Lemma 2.11. Let $X$ be a complex algebraic manifold. For every $p \geq 0$, there is an isomorphism of chain complexes

$$
\gamma_{1}: Z^{p}(X, *)_{0} \otimes \mathbb{R} \stackrel{\cong}{\longrightarrow} \mathscr{H}^{p}(X, *)_{0}
$$

sending $z$ to $\operatorname{cl}(z)$.

Proof. It follows from the isomorphism (1.30).

Remark 2.12. Observe that the complex $\mathscr{H}^{p}(X, *)_{0}$ has the same functorial properties as $Z^{p}(X, *)_{0} \otimes \mathbb{R}$.

Let $\mathscr{D}_{\mathbb{A}, Z^{p}}^{*, *}(X, p)_{0}$ be the 2 -iterated cochain complex, whose component of bidegree $(r,-n)$ is

$$
\tau \mathscr{D}_{\log , \mathcal{Z}_{n}^{p}}^{r}\left(X \times \square^{n}, p\right)_{0}=N \tau \mathcal{D}_{\log , Z_{n}^{p}}^{r}\left(X \times \square^{n}, p\right)=N \tau_{\leq 2 p} \mathscr{D}_{\log , Z_{n}^{p}}^{r}\left(X \times \square^{n}, p\right),
$$

and whose differentials are $\left(d_{\mathscr{D}}, \delta\right)$. As usual, we denote by $\mathscr{D}_{\mathbb{A}, \mathcal{Z}^{p}}^{*}(X, p)_{0}$ the associated simple complex and by $d_{s}$ its differential.

Let $\mathscr{D}_{\mathbb{A}, Z^{p}}^{2 p-*}(X, p)_{0}$ be the chain complex whose $n$-graded piece is $\mathscr{D}_{\mathbb{A}, Z^{p}}^{2 p-n}(X, p)_{0}$. 
Proposition 2.13. For every $p \geq 0$, the family of morphisms

$$
\begin{aligned}
\mathcal{D}_{\mathbb{A}, Z^{p}}^{2 p-n}(X, p)_{0} & \stackrel{\gamma_{1}^{\prime}}{\longrightarrow} \mathscr{H}^{p}(X, n)_{0}, \\
\left(\left(\omega_{n}, g_{n}\right), \ldots,\left(\omega_{0}, g_{0}\right)\right) & \longmapsto\left[\left(\omega_{n}, g_{n}\right)\right]
\end{aligned}
$$

defines a quasi-isomorphism of chain complexes between $\mathscr{D}_{\mathbb{A}, Z^{p}}^{2 p-*}(X, p)_{0}$ and $\mathscr{H}^{p}(X, *)_{0}$.

Proof. The map is well defined because $\left(\omega_{n}, g_{n}\right) \in \tau \mathcal{D}_{\log , \mathcal{Z}_{n}^{p}}^{2 p}\left(X \times \square^{n}, p\right)_{0}$. Therefore, by definition of the truncated complex $\left(\omega_{n}, g_{n}\right)$ is closed. To see that it is a morphism of complexes we compute

$$
\begin{aligned}
\gamma_{1}^{\prime} d_{s}\left(\left(\omega_{n}, g_{n}\right), \ldots,\left(\omega_{0}, g_{0}\right)\right) & =\gamma_{1}^{\prime}\left((-1)^{2 p} \delta\left(\omega_{n}, g_{n}\right)+d_{\mathscr{D}}\left(\omega_{n-1}, g_{n-1}\right), \ldots\right) \\
& =\left[\delta\left(\omega_{n}, g_{n}\right)+d_{\mathscr{D}}\left(\omega_{n-1}, g_{n-1}\right)\right]=\delta\left[\left(\omega_{n}, g_{n}\right)\right] .
\end{aligned}
$$

Now we consider the second quadrant spectral sequence with $E_{1}$-term

$$
E_{1}^{-n, r}=H^{r}\left(\tau \mathcal{D}_{\log , Z^{p}}^{*}\left(X \times \square^{n}, p\right)_{0}\right) .
$$

By construction, $E_{1}^{-n, r}=0$ for all $r>2 p$. Moreover, for all $r<2 p$ and for all $n$, the semipurity property of Deligne-Beilinson cohomology implies that

$$
H^{r}\left(\tau D_{\log , Z^{p}}^{*}\left(X \times \square^{n}, p\right)\right)=0 .
$$

Hence, by Proposition 1.24, the same is true for the normalized chain complex

$$
H^{r}\left(\tau \mathcal{D}_{\log , Z^{p}}^{*}\left(X \times \square^{n}, p\right)_{0}\right)=0, \quad r<2 p .
$$

Therefore, the $E_{1}$-term of the spectral sequence is

$$
E_{1}^{-n, r}= \begin{cases}0 & \text { if } r \neq 2 p, \\ H^{2 p}\left(\tau \mathcal{D}_{\log , Z^{p}}^{*}\left(X \times \square^{n}, p\right)_{0}\right) & \text { if } r=2 p .\end{cases}
$$

Finally, from Proposition 1.25, it follows that the natural map

$$
H^{2 p}\left(\tau \mathscr{D}_{\log , Z^{p}}^{*}\left(X \times \square^{n}, p\right)_{0}\right) \rightarrow \mathscr{H}^{p}(X, n)_{0}
$$

is an isomorphism. Using the explicit description of the spectral sequence associated to a double complex, it is clear that the morphism induced in cohomology by $\gamma_{1}^{\prime}$ agrees with the morphism induced by the spectral sequence. Hence the proposition is proved.

We denote

$$
\mathrm{CH}^{p}(X, n)_{\mathbb{R}}=\mathrm{CH}^{p}(X, n) \otimes \mathbb{R} .
$$


Corollary 2.15. Let $z \in \mathrm{CH}^{p}(X, n)_{\mathbb{R}}$ be the class of an algebraic cycle $z$ in $X \times \square^{n}$. By the isomorphisms of Lemma 2.11 and Proposition 2.13, the algebraic cycle $z$ is represented, in $H^{2 p-n}\left(\mathscr{D}_{\mathbb{A}, Z^{p}}(X, p)_{0}\right)$, by any cycle

$$
\left(\left(\omega_{n}, g_{n}\right), \ldots,\left(\omega_{0}, g_{0}\right)\right) \in \mathfrak{D}_{\mathbb{A}, Z^{p}}^{2 p-n}(X, p)_{0}
$$

such that

$$
\operatorname{cl}(z)=\left[\left(\omega_{n}, g_{n}\right)\right]
$$

Remark 2.16. Our construction differs from the construction given by Bloch in [4] in two points:

- He considered the 2-iterated complex of differential forms on the simplicial scheme $\mathbb{A}^{n}$, instead of the differential forms on the cubical scheme $\square^{n}$.

- In order to ensure the convergence of the spectral sequence in the proof of last proposition, he truncated the 2 -iterated complex in the direction given by the affine schemes.

2.6. Functoriality of $\mathscr{D}_{\mathbb{A}, \mathcal{Z}^{p}}^{*}(X, p)_{0}$. In many ways, the complex $\mathscr{D}_{\mathbb{A}, Z^{p}}^{*}(X, p)_{0}$ behaves like the complex $Z^{*}(X, *)_{0}$.

Lemma 2.17. Let $f: X \rightarrow Y$ be a flat map between two equidimensional complex algebraic manifolds. Then there is a pull-back map

$$
f^{*}: \mathscr{D}_{\mathbb{A}, Z^{p}}^{*}(Y, p)_{0} \rightarrow \mathscr{D}_{\mathbb{A}, Z^{p}}^{*}(X, p)_{0} .
$$

Proof. We will see that in fact there is a map of iterated complexes

$$
f^{*}: \mathscr{D}_{\mathbb{A}, Z^{p}}^{r,-n}(Y, p) \rightarrow \mathscr{D}_{\mathbb{A}, Z^{p}}^{r,-n}(X, p) .
$$

Let $Z$ be a codimension $p$ subvariety of $Y \times \square^{n}$ intersecting properly the faces of $\square^{n}$. Since $f$ is flat, there is a well-defined cycle $f^{*}(Z)$. It is a codimension $p$ cycle of $X \times \square^{n}$ intersecting properly the faces of $\square^{n}$, and whose support is $f^{-1}(Z)$. Then, by [14], 1.3.3, the pull-back of differential forms gives a morphism

$$
\tau \mathscr{D}_{\log }^{*}\left(Y \times \square^{n} \backslash Z, p\right) \stackrel{f^{*}}{\longrightarrow} \tau D_{\log }^{*}\left(X \times \square^{n} \backslash f^{-1}(Z), p\right) .
$$

Hence, there is an induced morphism

$$
\begin{aligned}
\tau \mathscr{D}_{\log }^{*}\left(Y \times \square^{n} \backslash \mathcal{Z}_{Y}^{p}, p\right) \stackrel{f^{*}}{\longrightarrow} \lim _{\underset{Z \in Z_{Y}^{p}}{\rightarrow} \tau D_{\log }^{*}\left(X \times \square^{n} \backslash f^{-1}(Z), p\right)} \longrightarrow \tau \mathscr{D}_{\log }^{*}\left(X \times \square^{n} \backslash Z_{X}^{p}, p\right),
\end{aligned}
$$

and thus, there is a pull-back morphism

$$
f^{*}: \mathscr{D}_{\mathbb{A}, Z^{p}}^{*,-n}(Y, p) \rightarrow \mathscr{D}_{\mathbb{A}, Z^{p}}^{*,-n}(X, p)
$$

compatible with the differential $\delta$. 
Remark 2.18. The pull-back defined here agrees with the pull-back defined by Bloch under the isomorphisms of Lemma 2.11 and Proposition 2.13. Indeed, let $f: X \rightarrow Y$ be a flat map. Then, if $Z$ is an irreducible subvariety of $Y$ and $(\omega, g)$ a couple representing the class of $[Z]$ in the Deligne-Beilinson cohomology with support, then the couple $\left(f^{*} \omega, f^{*} g\right)$ represents the class of $\left[f^{*}(Z)\right]$ (see [14], Theorem 3.6.1).

Proposition 2.19. Let $f: X \rightarrow Y$ be a morphism of equidimensional complex algebraic manifolds. Let $\mathcal{Z}_{f}^{p}$ be the subset consisting of the subvarieties $Z$ of $Y \times \square^{n}$ intersecting properly the faces of $\square^{n}$ and such that $X \times Z \times \square^{n}$ intersects properly the graph of $f, \Gamma_{f}$. Then

(i) the complex $\mathscr{D}_{\mathbb{A}, Z_{f}^{p}}^{*}(Y, p)_{0}$ is quasi-isomorphic to $\mathscr{D}_{\mathbb{A}, Z^{p}}^{*}(Y, p)_{0}$;

(ii) there is a well-defined pull-back

$$
f^{*}: \mathscr{D}_{\mathbb{A}, Z_{f}^{p}}^{*}(Y, p)_{0} \rightarrow \mathscr{D}_{\mathbb{A}, Z^{p}}^{*}(X, p)_{0} .
$$

Proof. Arguing as in the proof of the previous proposition, there is a pull-back map

$$
f^{*}: \tau \mathscr{D}_{\log }^{*}\left(Y \times \square^{n} \backslash \mathcal{Z}_{f}^{p}, p\right) \stackrel{f^{*}}{\longrightarrow} \tau \mathcal{D}_{\log }^{*}\left(X \times \square^{n} \backslash \mathcal{Z}^{p}, p\right),
$$

inducing a morphism

$$
f^{*}: \mathscr{D}_{\mathbb{A}, Z_{f}^{p}}^{*}(Y, p) \rightarrow D_{\mathbb{A}, Z^{p}}^{*}(X, p),
$$

and hence a morphism

$$
f^{*}: \mathscr{D}_{\mathbb{A}, Z_{f}^{p}}^{*}(Y, p)_{0} \rightarrow \mathscr{D}_{\mathbb{A}, Z^{p}}^{*}(X, p)_{0} .
$$

All that remains to be shown is that the inclusion

$$
\mathbb{D}_{\mathbb{A}, Z_{f}^{p}}^{*}(Y, p)_{0} \stackrel{i}{\rightarrow} \mathscr{D}_{\mathbb{A}, Z^{p}}^{*}(Y, p)_{0}
$$

is a quasi-isomorphism. By the quasi-isomorphism mentioned in Paragraph 2.2 and the quasi-isomorphism of Proposition 2.13, there is a commutative diagram

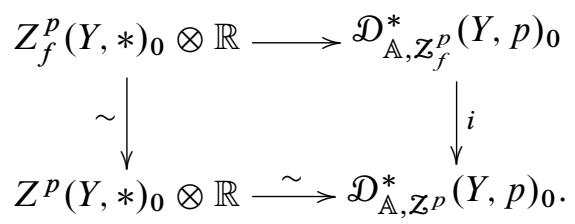

The proof that the upper horizontal arrow is a quasi-isomorphism is analogous to the proof of Proposition 2.13. Thus, we deduce that $i$ is a quasi-isomorphism. 


\section{Algebraic cycles and the Beilinson regulator}

In this section we define a chain morphism, in the derived category of chain complexes, that induces in homology the Beilinson regulator.

The construction is analogous to the definition of the cycle class map given by Bloch in [4], with the minor modifications mentioned in 2.16. However, in [4] there is no proof of the fact that the composition of the isomorphism $K_{n}(X)_{\mathbb{Q}} \cong$ $\bigoplus_{p \geq 0} \mathrm{CH}^{p}(X, n)_{\mathbb{Q}}$ with the cycle class map agrees with the Beilinson regulator.

3.1. Definition of the regulator. Consider the map of iterated cochain complexes defined by the projection onto the first factor

$$
\begin{aligned}
\mathscr{D}_{\mathbb{A}, Z^{p}}^{r,-n}(X, p) & \\
=\tau_{\leq 2 p} s\left(D_{\log }^{*}\left(X \times \square^{n}, p\right) \rightarrow \mathcal{D}_{\log }^{*}\left(X \times \square^{n} \backslash \mathcal{Z}^{p}, p\right)\right)^{r} & \stackrel{\rho}{\longrightarrow} \tau \mathcal{D}_{\log }^{r}\left(X \times \square^{n}, p\right), \\
(\omega, g) & \longmapsto \omega .
\end{aligned}
$$

It induces a cochain morphism

$$
\mathscr{D}_{\mathbb{A}, Z^{p}}^{*}(X, p)_{0} \stackrel{\rho}{\longrightarrow} D_{\mathbb{A}}^{*}(X, p)_{0},
$$

and hence a chain morphism

$$
\mathscr{D}_{\mathbb{A}, Z^{p}}^{2 p-*}(X, p)_{0} \stackrel{\rho}{\longrightarrow} \mathscr{D}_{\mathbb{A}}^{2 p-*}(X, p)_{0} .
$$

The morphism induced by $\rho$ in homology, together with the isomorphisms of Propositions $2.8,2.11$ and 2.13, induce a morphism

$$
\rho: \mathrm{CH}^{p}(X, n) \rightarrow \mathrm{CH}^{p}(X, n)_{\mathbb{R}} \rightarrow H_{\mathscr{D}}^{2 p-n}(X, \mathbb{R}(p)) .
$$

By abuse of notation, it will also be denoted by $\rho$.

By Corollary 2.15, we deduce that, if $z \in Z^{p}(X, n)_{0}$, then

$$
\rho(z)=\left(\omega_{n}, \ldots, \omega_{0}\right),
$$

for any cycle $\left(\left(\omega_{n}, g_{n}\right), \ldots,\left(\omega_{0}, g_{0}\right)\right) \in \mathscr{D}_{\mathbb{A}, Z^{p}}^{2 p-n}(X, p)_{0}$ such that $\left[\left(\omega_{n}, g_{n}\right)\right]=\operatorname{cl}(z)$.

Proposition 3.3. (i) The morphism $\rho: \mathscr{D}_{\mathbb{A}, Z^{p}}^{2 p-*}(X, p)_{0} \rightarrow \mathscr{D}_{\mathbb{A}}^{2 p-*}(X, p)_{0}$ is contravariant for flat maps.

(ii) The induced morphism $\rho: \mathrm{CH}^{p}(X, n) \rightarrow H_{D}^{2 p-n}(X, \mathbb{R}(p))$ is contravariant for arbitrary maps.

Proof. Both assertions are obvious. Let

$$
z=\left(\left(\omega_{n}, g_{n}\right), \ldots,\left(\omega_{0}, g_{0}\right)\right) \in \mathscr{D}_{\mathbb{A}, \mathcal{Z}^{p}}^{2 p-n}(X, p)_{0}
$$


be a cycle such that its inverse image by $f$ is defined. This is the case when $f$ is flat or when $z$ belongs to $\mathscr{D}_{\mathbb{A}, \mathcal{Z}_{f}^{p}}^{2 p-*}(X, p)_{0}$. In both cases

$$
f^{*}\left(\left(\omega_{n}, g_{n}\right), \ldots,\left(\omega_{0}, g_{0}\right)\right)=\left(\left(f^{*} \omega_{n}, f^{*} g_{n}\right), \ldots,\left(f^{*} \omega_{0}, f^{*} g_{0}\right)\right)
$$

and the claim follows.

Remark 3.4. Let $X$ be an equidimensional compact complex algebraic manifold. Observe that, by definition, the morphism

$$
\rho: \mathrm{CH}^{p}(X, 0)=\mathrm{CH}^{p}(X) \rightarrow H_{D}^{2 p}(X, \mathbb{R}(p))
$$

agrees with the cycle class map cl.

Now let $E$ be a vector bundle of rank $n$ over $X$. For every $p=1, \ldots, n$, there exists a characteristic class $C_{p}^{\mathrm{CH}}(E) \in \mathrm{CH}^{p}(X)$ (see [17]) and a characteristic class $C_{p}^{\mathscr{D}}(E) \in H_{\mathscr{D}}^{2 p}(X, \mathbb{R}(p))$, called the $p$-th Chern class of the vector bundle $E$. By definition, $\operatorname{cl}\left(C_{p}^{\mathrm{CH}}(E)\right)=C_{p}^{\mathbb{D}}(E)$. Hence,

$$
\rho\left(C_{p}^{\mathrm{CH}}(E)\right)=C_{p}^{D}(E),
$$

for all $p=1, \ldots, n$.

3.2. Comparison with the Beilinson regulator. We prove here that the regulator defined in (3.2) agrees with the Beilinson regulator.

The comparison is based on the following facts:

- The morphism $\rho$ is compatible with inverse images.

- The morphism $\rho$ is defined for quasi-projective schemes.

In view of these properties, it is enough to prove that the two regulators agree when $X$ is a Grassmanian manifold, which in turn follows from Remark 3.4.

Theorem 3.5. Let $X$ be an equidimensional complex algebraic scheme. Let $\rho^{\prime}$ be the composition of $\rho$ with the isomorphism given by the Chern character

$$
\rho^{\prime}: K_{n}(X)_{\mathbb{Q}} \stackrel{\cong}{\rightarrow} \bigoplus_{p \geq 0} \mathrm{CH}^{p}(X, n)_{\mathbb{Q}} \stackrel{\rho}{\rightarrow} \bigoplus_{p \geq 0} H_{\mathscr{D}}^{2 p-n}(X, \mathbb{R}(p)) .
$$

Then the morphism $\rho^{\prime}$ agrees with the Beilinson regulator.

Proof. The outline of the proof is as follows. We first recall the description of the Beilinson regulator in terms of homotopy theory of simplicial sheaves as in [15]. Then we recall the construction of the Chern character given by Bloch. We proceed reducing the comparison of the two maps to the case $n=0$ and for $X$ a Grassmanian 
scheme. We finally prove that at this stage both maps agree. Our site will always be the small Zariski site over $X$.

Consider $X$ as a smooth quasi-projective variety over $\mathbb{C}$. Let $B \cdot \mathrm{GL}_{N}$ be the simplicial version of the classifying space of the group $\mathrm{GL}_{N}(\mathbb{C})$ viewed as a simplicial complex manifold. Recall that all the face morphisms are flat. Let $B . \mathrm{GL}_{N, X}$ be the simplicial sheaf over $X$ given by the sheafification of the presheaf

$$
U \mapsto B . \mathrm{GL}_{N}\left(\Gamma\left(U, \mathcal{O}_{U}\right)\right)
$$

for every Zariski open $U \subseteq X$. This is the same as the simplicial sheaf given by

$$
U \mapsto \underline{\operatorname{Hom}}\left(U, B . \mathrm{GL}_{N}\right),
$$

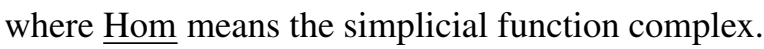
let

Consider the inclusion morphisms $B \cdot \mathrm{GL}_{N, X} \rightarrow B \cdot \mathrm{GL}_{N+1, X}$, for all $N \geq 1$, and

$$
\text { B.GL } \operatorname{Gim}_{X} B . \mathrm{GL}_{N, X} .
$$

Let $\mathbb{Z}_{\infty} B$.GL $\mathrm{G}_{N, X}$ and $\mathbb{Z}_{\infty} B$.GL $\mathrm{GL}_{X}$ be the sheaves associated to the respective Bousfield-Kan completions. Finally, let $\mathbb{Z}$ be the constant simplicial sheaf on $\mathbb{Z}$ and consider the following sheaves on $X$

$$
\begin{aligned}
\mathbb{K}_{X} & =\mathbb{Z} \times \mathbb{Z}_{\infty} B . \mathrm{GL}_{X}, \\
\mathbb{K}_{X}^{N} & =\mathbb{Z} \times \mathbb{Z}_{\infty} B . \mathrm{GL}_{N, X} .
\end{aligned}
$$

By [15], Proposition 5, there is a natural isomorphism

$$
K_{m}(X) \cong H^{-m}\left(X, \mathbb{K}_{X}\right)=\lim _{\vec{N}} H^{-m}\left(X, \mathbb{K}_{X}^{N}\right) .
$$

Here $H^{-*}(\cdot, *)$ denotes the generalized cohomology with coefficients in $\mathbb{K}_{X}$ and $\mathbb{K}_{X}^{N}$, as described in [15].

The Beilinson regulator is the Chern character taking values in Deligne-Beilinson cohomology. The regulator can be described in terms of homotopy theory of sheaves as follows.

Consider the Dold-Puppe functor $\mathcal{K} .(\cdot)$ (see [12]), which associates to every cochain complex of abelian groups concentrated in non-positive degrees, $G^{*}$, a simplicial abelian group $\mathcal{K} .(G)$, pointed by zero. It satisfies the property that $\pi_{i}(\mathcal{K} .(G), 0)=H^{-i}\left(G^{*}\right)$.

In [13], Gillet constructs Chern classes

$$
C_{p}^{D} \in H^{2 p}\left(B . \mathrm{GL}_{N}, \mathbb{R}(p)\right), \quad N \gg 0,
$$

which induce morphisms

$$
c_{p, X}^{\mathcal{D}}: \mathbb{K}_{X, \cdot}^{N} \rightarrow \mathcal{K} .\left(\mathscr{D}_{X}^{*}(\cdot, p)[2 p]\right), \quad N \gg 0
$$


in the homotopy category of simplicial sheaves.

These morphisms are compatible with the morphisms $\mathbb{K}_{X, \cdot}^{N} \rightarrow \mathbb{K}_{X, \cdot}^{N+1}$. Therefore, we obtain a morphism

$$
K_{m}(X)=\lim _{\vec{N}} H^{-m}\left(X, \mathbb{K}_{X}^{N}\right) \stackrel{C_{p, X}^{\mathcal{D}}}{\longrightarrow} H_{\mathscr{D}}^{2 p-m}(X, \mathbb{R}(p)) .
$$

Using the standard formula for the Chern character in terms of the Chern classes, we obtain a morphism

$$
K_{m}(X) \stackrel{c h^{\mathscr{D}}}{\longrightarrow} H_{\mathscr{D}}^{2 p-m}(X, \mathbb{R}(p)),
$$

which is the Beilinson regulator.

The Chern character for higher Chow groups. The description of the isomorphism $K_{n}(X)_{\mathbb{Q}} \stackrel{\cong}{\rightarrow} \bigoplus_{p \geq 0} \mathrm{CH}^{p}(X, n)_{\mathbb{Q}}$ given by Bloch follows the same pattern as the description of the Beilinson regulator. However, since the complexes that define the higher Chow groups are not sheaves (in fact not even functors) on the big Zariski site, a few modifications are necessary. We give here a sketch of the construction. For details see [3].

If $Y$. is a simplicial scheme whose face maps are flat, then there is a well-defined 2-iterated cochain complex $Z^{p}(Y ., *)_{0}$, whose $(n, m)$-bigraded group is

$$
Z^{p}\left(Y_{-n}, m\right)_{0},
$$

and induced differentials. The higher algebraic Chow groups of $Y$. are then defined as

$$
\mathrm{CH}^{p}(Y ., n)=H^{n}\left(Z^{p}(Y ., *)_{0}\right) .
$$

Since the face maps of the simplicial scheme $B . \mathrm{GL}_{N}$ are flat it follows that the group $\mathrm{CH}^{p}\left(B . \mathrm{GL}_{N}, n\right)$ is well defined for every $p$ and $n$.

First, Bloch constructs universal Chern classes

$$
C_{p}^{\mathrm{CH}} \in \mathrm{CH}^{p}\left(B . \mathrm{GL}_{N}, 0\right),
$$

following the ideas of Gillet. These classes are represented by elements

$$
C_{p}^{\mathrm{CH}, i} \in Z^{p}\left(B_{i} \mathrm{GL}_{N}, i\right)_{0} .
$$

Because at the level of complexes the pull-back morphism is not defined for arbitrary maps, one cannot consider the pull-back of these classes $C_{p}^{\mathrm{CH}, i}$ to $X$, as was the case for the Beilinson regulator. However, by [3], §7, there exists a purely transcendental extension $L$ of $\mathbb{C}$, and classes $C_{p}^{\mathrm{CH}, i}$ defined over $L$, such that the pull-back $f^{*} C_{p}^{\mathrm{CH}, i}$ is defined for every $\mathbb{C}$-morphism $f: V \rightarrow B_{i} \mathrm{GL}_{N}$.

Then there is a map of simplicial Zariski sheaves on $X$

$$
B . \mathrm{GL}_{N, X} \rightarrow \mathcal{K}_{X}\left(g_{*} Z_{X_{L}}^{p}(-, *)_{0}\right),
$$


where $g: X_{L} \rightarrow X$ is the natural map obtained by extension to $L$.

There is a specialization process described in [3], which, in the homotopy category of simplicial sheaves over $X$, gives a well-defined map

$$
\mathcal{K}_{X}\left(g_{*} Z_{X_{L}}^{p}(-, *)_{0}\right) \rightarrow \mathcal{K}_{X}\left(Z_{X}^{p}(-, *)_{0}\right)
$$

Therefore, there are maps $C_{p, X}^{\mathrm{CH}} \in\left[B \cdot \mathrm{GL}_{N, X}, \mathcal{K}_{X}\left(Z_{X}^{*}(\cdot, p)\right)\right]$, where $[\cdot, \cdot]$ denotes the set of arrows in the homotopy category. Proceeding as above, we obtain the Chern character morphism

$$
K_{m}(X) \rightarrow \bigoplus_{p \geq 0} \mathrm{CH}^{p}(X, m)_{\mathbb{Q}}
$$

For $m=0$, this is the usual Chern character.

End of the proof. Since, at the level of complexes, $\rho$ is functorial for flat maps, there is a sheaf map

$$
\rho: \mathcal{K}_{X}\left(Z_{X}^{*}(\cdot, p)\right) \rightarrow \mathcal{K} .\left(\mathcal{D}_{\log }(X, p)\right)
$$

in the small Zariski site of $X$.

It follows that the composition $\rho \circ C_{p}^{\mathrm{CH}}$ is obtained by the same procedure as the Beilinson regulator, but starting with the characteristic classes $\rho\left(C_{p}^{\mathrm{CH}}\right) \in$ $H_{\mathscr{D}}^{2 p}(X, \mathbb{R}(p))$ instead of the classes $C_{p}^{\mathscr{D}}$. Therefore, it remains to see that

$$
\rho\left(C_{p}^{\mathrm{CH}}\right)=C_{p}^{\mathfrak{D}}
$$

For integers $N, k \geq 0$ let $\operatorname{Gr}(N, k)$ be the complex Grassmanian scheme of $N$ planes in $\mathbb{C}^{k}$. It is a smooth complex projective scheme. Let $E_{N, k}$ be the rank $N$ universal bundle of $\operatorname{Gr}(N, k)$ and $U_{k}=\left(U_{k, \alpha}\right)_{\alpha}$ its standard trivialization. Let $N . U_{k}$ denote the nerve of this cover. It is a hypercover of $\operatorname{Gr}(N, k), N \cdot U_{k} \stackrel{\pi}{\rightarrow} \operatorname{Gr}(N, k)$. Consider the classifying map of the vector bundle $E_{N, k}$,

$$
\varphi_{k}: N . U_{k} \rightarrow B \cdot \mathrm{GL}_{N}
$$

which satisfies $\pi^{*}\left(E_{N, k}\right)=\varphi_{k}^{*}\left(E_{.}^{N}\right)$, for $E_{\text {. }}^{N}$ the universal vector bundle over B.GL ${ }_{N}$. Observe that all the faces and degeneracy maps of the simplicial scheme $N . U_{k}$ are flat, as well as the inclusion maps $N_{l} U_{k} \rightarrow \operatorname{Gr}(N, k)$. Thus $\mathrm{CH}^{p}\left(N . U_{k}, m\right)$ is defined and there is a pull-back map

$$
\mathrm{CH}^{p}(\mathrm{Gr}(N, k), m) \stackrel{\pi^{*}}{\rightarrow} \mathrm{CH}^{p}\left(N . U_{k}, m\right) .
$$

Since $\rho$ is defined on $N . U_{k}$ and is a functorial map, we obtain the following 
commutative diagram:

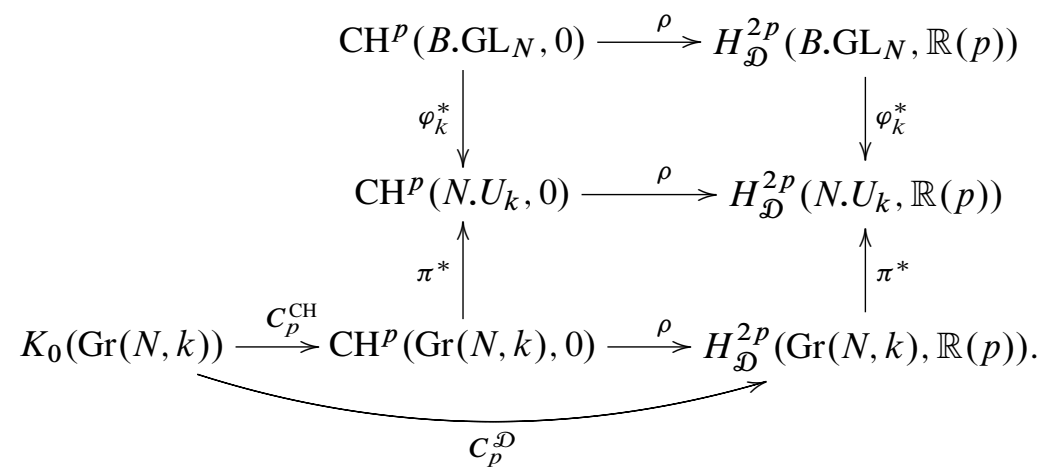

By construction, $C_{p}^{\mathrm{CH}}\left(E_{N, k}\right)$ is the standard $p$-th Chern class in the classical Chow group of $\operatorname{Gr}(N, k)$, and $C_{p}^{D}\left(E_{N, k}\right)$ is the $p$-th Chern class in Deligne-Beilinson cohomology. It then follows from Remark 3.4 that

$$
\rho\left(C_{p}^{\mathrm{CH}}\left(E_{N, k}\right)\right)=C_{p}^{\mathcal{D}}\left(E_{N, k}\right) .
$$

The vector bundle $E_{N, k} \in K_{0}(\operatorname{Gr}(N, k))=\lim _{\vec{M}}\left[\operatorname{Gr}(N, k), \mathbb{K}^{M}\right]$ is represented, in the homotopy category of simplicial sheaves, by the diagram

$$
\operatorname{Gr}(N, k) \stackrel{\pi}{\leftarrow} N \cdot U_{k} \stackrel{\varphi_{k}}{\longrightarrow} B . \mathrm{GL}_{N},
$$

where the map $\pi$ is a weak equivalence of sheaves because $N . U_{k}$ is a hypercover of $\operatorname{Gr}(N, k)$. This means that

$$
\varphi_{k}^{*}\left(C_{p}^{\mathrm{CH}}\left(E_{.}^{N}\right)\right)=\pi^{*}\left(C_{p}^{\mathrm{CH}}\left(E_{N, k}\right)\right) .
$$

Also, since $\pi$ is an hypercover, $\pi^{*}$ is an isomorphism in Deligne-Beilinson cohomology. Moreover, for each $m_{0}$, there exists $k_{0}$ such that, if $m \leq m_{0}$ and $k \geq k_{0}$, $\varphi_{k}^{*}$ is an isomorphism on the cohomology group $H_{\mathscr{D}}^{2 m}(, \mathbb{R}(m))$. To see this, we first use the computation of the mixed Hodge structure of the cohomology of the classifying space given in [11] and the well known mixed Hodge structure of the cohomology of the Grassmanian manifolds to reduce it to a comparison at the level of singular cohomology. Then we use that the infinite Grassmanian is homotopically equivalent to the classifying space. Finally we use the cellular decomposition of the infinite Grassmanian to compare its cohomology with the cohomology of the finite Grassmanian (see for instance [22]).

Under these isomorphisms, we obtain the equality

$$
C_{p}^{\mathbb{D}}\left(E_{N, k}\right)=\left(\pi^{*}\right)^{-1} \varphi_{k}^{*}\left(C_{p}^{\mathscr{D}}\left(E_{.}^{N}\right)\right) .
$$


Hence,

$$
\begin{aligned}
\rho\left(C_{p}^{\mathrm{CH}}\left(E_{.}^{N}\right)\right)=C_{p}^{\mathcal{D}}\left(E_{.}^{N}\right) & \Longleftrightarrow \varphi_{k}^{*} \rho\left(C_{p}^{\mathrm{CH}}\left(E_{\cdot}^{N}\right)\right)=\varphi_{k}^{*} C_{p}^{\mathcal{D}}\left(E_{.}^{N}\right) \\
& \Longleftrightarrow \rho \varphi_{k}^{*}\left(C_{p}^{\mathrm{CH}}\left(E_{.}^{N}\right)\right)=\varphi_{k}^{*} C_{p}^{\mathscr{D}}\left(E_{.}^{N}\right) .
\end{aligned}
$$

The last equality follows directly from (3.7), (3.8) and (3.9). Therefore, the theorem is proved.

\section{Higher arithmetic Chow groups}

Let $X$ be an arithmetic variety over a field. Using the description of the Beilinson regulator given in Section 3, we define the higher arithmetic Chow groups, $\widehat{\mathrm{CH}}^{n}(X, p)$. The definition is analogous to the definition given by Goncharov, in [16], but using differential forms instead of currents.

We need to restrict ourselves to arithmetic varieties over a field, because the theory of higher algebraic Chow groups by Bloch is only well established for schemes over a field. That is, we can define the higher arithmetic Chow groups for arbitrary arithmetic varieties, but since the functoriality properties and the product structure of the higher algebraic Chow groups are described only for schemes over a field, we cannot give a product structure or define functoriality for the higher arithmetic Chow groups of arithmetic varieties over a ring. Note however that, using work by Levine [21], it should be possible to extend the constructions here to smooth varieties over a Dedekind domain, at least after tensoring with $\mathbb{Q}$. In fact, when extending the definition to arithmetic varieties over a ring, it might be better to use the point of view of motivic homology à la Voevodsky or any of its more recent variants.

4.1. Higher arithmetic Chow groups. Following [14], an arithmetic field is a triple $\left(K, \Sigma, F_{\infty}\right)$, where $K$ is a field, $\Sigma$ is a nonempty set of complex immersions $K \hookrightarrow \mathbb{C}$ and $F_{\infty}$ is a conjugate-linear $\mathbb{C}$-algebra automorphism of $\mathbb{C}^{\Sigma}$ that leaves invariant the image of $K$ under the diagonal immersion. By an arithmetic variety $X$ over the arithmetic ring $K$ we mean a regular quasi-projective $K$-scheme $X$.

To the arithmetic variety $X$ we associate a complex variety $X_{\mathbb{C}}=\bigsqcup_{\iota \in \Sigma} X_{\iota}$, and a real variety $X_{\mathbb{R}}=\left(X_{\mathbb{C}}, F_{\infty}\right)$. The Deligne complex of differential forms on $X$ is defined from the real variety $X_{\mathbb{R}}$ as

$$
\mathscr{D}_{\log }^{n}(X, p):=\mathscr{D}_{\log }^{n}\left(X_{\mathbb{C}}, p\right)^{\sigma=\mathrm{id}},
$$

where $\sigma$ is the involution as in Paragraph 1.10. We define analogously the chain complexes

$$
\mathscr{D}_{\mathbb{A}}^{2 p-*}(X, p)_{0}, \quad \mathscr{D}_{\mathbb{A}}^{2 p-*}(X, p)_{00}, \quad \mathscr{D}_{\mathbb{A}, Z^{p}}^{2 p-*}(X, p)_{0}, \quad \text { and } \quad \mathscr{D}_{\mathbb{A}, Z^{p}}^{2 p-*}(X, p)_{00} .
$$


Let $\gamma_{1}$ be the composition

$\gamma_{1}: Z^{p}(X, n)_{0} \stackrel{\otimes \mathbb{R}}{\longrightarrow} Z^{p}(X, n)_{0} \otimes \mathbb{R} \stackrel{\times_{F} \mathbb{R}}{\longrightarrow} Z^{p}\left(X_{\mathbb{R}}, n\right)_{0} \otimes \mathbb{R} \cong \mathscr{H}^{p}(X, n)_{0}$.

We consider the diagram of complexes of the type of (1.12)

$$
\hat{Z}^{p}(X, *)_{0}=(\overbrace{Z^{p}(X, *)_{0}}^{\gamma_{1}} \overbrace{Z D_{\log }^{2 p}(X, p)_{*}}^{\mathscr{H}^{p}(X, *)_{0}}) \text {, }
$$

where $Z D_{\log }^{2 p}(X, p)_{*}$ is the chain complex which is zero in all degrees except in degree zero, where it consists of the vector subspace of cycles in $\mathscr{D}_{\log }^{2 p}(X, p)$. Note that it agrees with $Z E_{\log , \mathbb{R}}^{p, p}(X)(p)$, the subspace of $E_{\log , \mathbb{R}}^{p, p}(X)(p)$ consisting of differential forms with logarithmic singularities that are real up to a product by $(2 \pi i)^{p}$, of type $(p, p)$ and that vanish under $\partial$ and $\bar{\partial}$. The morphism $i$ is the inclusion of chain complexes.

Definition 4.2. The higher arithmetic Chow complex is the simple complex associated to the diagram $\widehat{Z}^{p}(X, *)_{0}$, as defined in (1.6):

$$
\widehat{Z}^{p}(X, *)_{0}:=s\left(\widehat{Z}^{p}(X, *)_{0}\right) .
$$

Recall that, by definition, $\widehat{Z}^{p}(X, n)_{0}$ consists of 5-tuples

$$
\begin{array}{r}
\left(Z, \alpha_{0}, \alpha_{1}, \alpha_{2}, \alpha_{3}\right) \in Z^{p}(X, n)_{0} \oplus \mathscr{D}_{\mathbb{A}, Z^{p}}^{2 p-n}(X, p)_{0} \oplus Z \mathscr{D}_{\log }^{2 p}(X, p)_{n} \\
\oplus \mathscr{H}^{p}(X, n+1)_{0} \oplus \mathscr{D}_{\mathbb{A}}^{2 p-n-1}(X, p)_{0},
\end{array}
$$

and the differential is given by

$$
\begin{aligned}
\hat{Z}^{p}(X, n)_{0} \stackrel{d}{\longrightarrow} \hat{Z}^{p}(X, n-1)_{0} & \\
\left(Z, \alpha_{0}, \alpha_{1}, \alpha_{2}, \alpha_{3}\right) \longmapsto & \left(\delta(Z), d_{s}\left(\alpha_{0}\right), 0, \gamma_{1}(Z)-\gamma_{1}^{\prime}\left(\alpha_{0}\right)-\delta\left(\alpha_{2}\right),\right. \\
& \left.\rho\left(\alpha_{0}\right)-\alpha_{1}-d_{s}\left(\alpha_{3}\right)\right) .
\end{aligned}
$$

Note that $\alpha_{1}$ will be zero unless $n=0$. Its differential, however, is always zero.

Definition 4.3. Let $X$ be an arithmetic variety over an arithmetic field. The $(p, n)$-th higher arithmetic Chow group of $X$ is defined by

$$
\widehat{\mathrm{CH}}^{p}(X, n):=H_{n}\left(\widehat{Z}^{p}(X, *)_{0}\right), \quad p, n \geq 0 .
$$


By its definition as the cohomology of a simple of a diagram of complexes it comes equipped with the following morphisms

$$
\begin{aligned}
\zeta: \widehat{\mathrm{CH}}^{p}(X, n) & \longrightarrow \mathrm{CH}^{p}(X, n), & \zeta\left[\left(Z, \alpha_{0}, \ldots, \alpha_{3}\right)\right]=[Z], \\
\mathrm{a}: H_{\mathscr{D}}^{2 p-n}(X, \mathbb{R}(p)) & \longrightarrow \widehat{\mathrm{CH}}^{p}(X, n), & \mathrm{a}([a])=[(0,0,0,0,-a)], \\
\mathrm{a}: \mathcal{D}_{\log }^{2 p-1}(X, p) & \longrightarrow \widehat{\mathrm{CH}}^{p}(X, 0), & \mathrm{a}(\tilde{a})=\left[\left(0,0,-d_{\mathscr{D}} a, 0,-a\right)\right], \\
\omega: \widehat{\mathrm{CH}}^{p}(X, 0) & \longrightarrow \mathrm{ZD}_{\log }^{2 p}(X, p), & \omega\left(\left[\left(Z, \alpha_{0}, \ldots, \alpha_{3}\right)\right]\right)=\alpha_{1} .
\end{aligned}
$$

Proposition 4.4. There is a long exact sequence

$$
\begin{gathered}
\cdots \rightarrow \widehat{\mathrm{CH}}^{p}(X, n) \stackrel{\zeta}{\rightarrow} \mathrm{CH}^{p}(X, n) \stackrel{\rho}{\rightarrow} H_{\mathscr{D}}^{2 p-n}(X, \mathbb{R}(p)) \stackrel{\text { a }}{\rightarrow} \widehat{\mathrm{CH}}^{p}(X, n-1) \rightarrow \cdots \\
\cdots \rightarrow \mathrm{CH}^{p}(X, 1) \stackrel{\rho}{\rightarrow} \mathscr{D}_{\log }^{2 p-1}(X, p) / \operatorname{im} d_{\mathscr{D}} \stackrel{\mathrm{a}}{\rightarrow} \widehat{\mathrm{CH}}^{p}(X, 0) \stackrel{\zeta}{\rightarrow} \mathrm{CH}^{p}(X, 0) \rightarrow 0,
\end{gathered}
$$

where $\rho$ is the Beilinson regulator.

Proof. It follows from Theorem 3.5, Lemma 1.16 and the fact that the homology groups of the complex

$$
s\left(Z \mathcal{D}_{\log }^{2 p}(X, p)_{*} \stackrel{i}{\rightarrow} \mathscr{D}_{\mathbb{A}}^{2 p-*}(X, p)_{0}\right)
$$

are $H_{\mathscr{D}}^{2 p-n}(X, \mathbb{R}(p))$ in degree $n \neq 0$ and $\mathscr{D}_{\log }^{2 p-1}(X, p) / \operatorname{im} d_{\mathscr{D}}$ in degree 0 .

Remark 4.6. Let $\widehat{D}_{\mathbb{A}}^{*, *}(X, p)_{0}$ be the 2 -iterated cochain complex given by the quotient $\mathscr{D}_{\mathbb{A}}^{*, *}(X, p)_{0} / \mathscr{D}^{2 p, 0}(X, p)$. That is, for all $r, n$,

$$
\widehat{D}_{\mathbb{A}}^{r,-n}(X, p)_{0}= \begin{cases}0 & \text { if } r=2 p \text { and } n=0, \\ \mathcal{D}_{\mathbb{A}}^{r,-n}(X, p)_{0} & \text { otherwise. }\end{cases}
$$

Let $\widehat{D}_{\mathbb{A}}^{*}(X, p)_{0}$ denote the simple complex associated to $\hat{D}_{\mathbb{A}}^{*, *}(X, p)_{0}$. Consider the composition of $\rho$ with the projection map

$$
\rho: \mathscr{D}_{\mathbb{A}, Z^{p}}^{2 p-*}(X, p)_{0} \stackrel{\rho}{\rightarrow} \mathscr{D}_{\mathbb{A}}^{2 p-*}(X, p)_{0} \rightarrow \widehat{D}_{\mathbb{A}}^{2 p-*}(X, p)_{0} .
$$

Then there is a diagram of chain complexes of the type of (1.9)

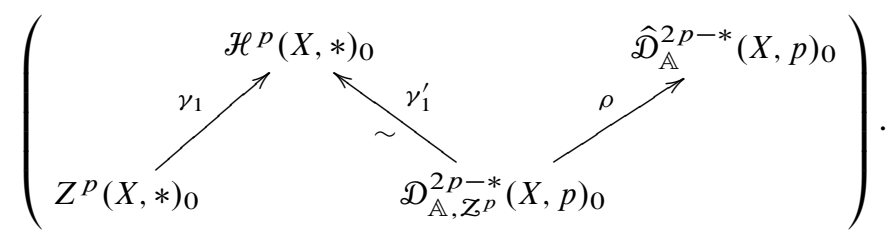


By Proposition 1.13, the simple complex associated to the diagram (4.7) is quasiisomorphic to the complex $\widehat{Z}^{p}(X, *)_{0}$ and hence, its homology groups are isomorphic $\widehat{\mathrm{CH}}^{p}(X, *)$. Nevertheless, in order to define a product structure in $\widehat{\mathrm{CH}}^{*}(X, *)$ it is better to work with the diagram (4.1).

4.2. Agreement with the arithmetic Chow groups. Let $X$ be an arithmetic variety and let $\widehat{\mathrm{CH}}^{p}(X)$ denote the $p$-th arithmetic Chow group of $X$ as defined by Burgos in [5]. We recall here its definition.

For every $p$, let $Z^{p}(X)=Z^{p}(X, 0)$ and let $Z \mathscr{D}_{\log }^{2 p}(X, p)$ denote the subgroup of cycles of $\mathscr{D}_{\log }^{2 p}(X, p)$. Let $\hat{Z}^{p}(X)$ denote the set

$$
\left\{(Z,(\omega, \tilde{g})) \in Z^{p}(X) \oplus Z \mathscr{D}_{\log }^{2 p}(X, p) \oplus \frac{\mathscr{D}_{\log }^{2 p-1}\left(X \backslash Z^{p}, p\right)}{\operatorname{im} d_{\mathscr{D}}} \mid \begin{array}{c}
\omega=d_{\mathscr{D}} \tilde{g} \\
\operatorname{cl}(Z)=[(\omega, g)]
\end{array}\right\} .
$$

If $Z \in Z^{p}(X)$, a Green form for $Z$ is a couple $(\omega, \tilde{g})$ as before such that $\operatorname{cl}(Z)=$ $[(\omega, g)]$, where $g$ is any representative of $\tilde{g}$.

Let $Y$ be a codimension $p-1$ subvariety of $X$ and let $f \in k^{*}(Y)$. As shown in [5], $\S 7$, there is a canonical Green form attached to $\operatorname{div} f$. It is denoted by $\mathrm{g}(f)$ and it is of the form $(0, \tilde{g}(f))$ for some class $\tilde{g}(f)$.

Let $\widehat{\operatorname{Rat}}^{p}(X)$ be the subgroup of $\widehat{Z}^{p}(X)$ generated by

$\left\{(\operatorname{div} f, \mathrm{~g}(f)) \mid f \in k^{*}(Y), Y \subset X\right.$ a codimension $p-1$ subvariety $\}$.

For every $p \geq 0$, the arithmetic Chow group of $X$ is defined by

$$
\widehat{\mathrm{CH}}^{p}(X)=\widehat{Z}^{p}(X) \widehat{\operatorname{Rat}}^{p}(X) .
$$

It is proved in [14], Theorem 3.3.5 and [5], Theorem 7.3, that these groups fit into exact sequences

$$
\mathrm{CH}^{p-1, p}(X) \stackrel{\rho}{\rightarrow} \mathcal{D}_{\log }^{2 p-1}(X, p) / \mathrm{im} d_{\mathscr{D}} \stackrel{\mathrm{a}}{\rightarrow} \widehat{\mathrm{CH}}^{p}(X) \stackrel{\zeta}{\rightarrow} \mathrm{CH}^{p}(X) \rightarrow 0
$$

where:

- $\mathrm{CH}^{p-1, p}(X)$ is the term $E_{2}^{p-1,-p}$ in the Quillen spectral sequence (see $\S 7$ of [23]).

- The map $\rho$ is the cycle class map and is the Beilinson regulator after composition with the isomorphism $K_{1}(X)_{\mathbb{Q}} \cong \bigoplus_{p \geq 0} \mathrm{CH}^{p-1, p}(X)_{\mathbb{Q}}$.

- The map $\zeta$ is the projection on the first component.

- The map a sends $\alpha$ to $\left(0,\left(-d_{\mathscr{D}} \alpha,-\alpha\right)\right)$. 
Theorem 4.8. The morphism

$$
\begin{aligned}
\widehat{\mathrm{CH}}^{p}(X) & \stackrel{\Phi}{\longrightarrow} \widehat{\mathrm{CH}}^{p}(X, 0), \\
{[(Z,(\omega, \tilde{g}))] } & \longmapsto[(Z,(\omega, g), 0,0,0)],
\end{aligned}
$$

where $g$ is any representative of $\tilde{g} \in \mathscr{D}_{\log }^{2 p-1}(X, p) / \mathrm{im} d_{\mathscr{D}}$, is an isomorphism.

Proof. We first prove that $\Phi$ is well defined. Afterwards, we will prove that the diagram

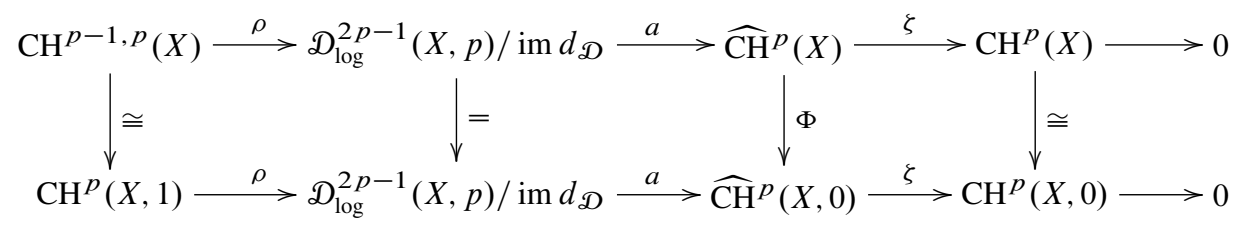

is commutative. The statement then follows from the five lemma.

The proof is a consequence of Lemmas 4.9, 4.10 and 4.11 below.

Lemma 4.9. The map $\Phi$ is well defined.

Proof. We have to prove that

(i) the elements in the image of $\Phi$ are indeed cycles in $\widehat{Z}^{p}(X, 0)_{0}$;

(ii) the map $\Phi$ does not depend on the choice of a representative of $g$;

(iii) the map $\Phi$ is zero on $\widehat{\operatorname{Rat}}^{p}(X)$.

Let $[(Z,(\omega, \tilde{g}))] \in \widehat{\mathrm{CH}}^{p}(X)$. The claim (i) follows from the equality $\operatorname{cl}(Z)=$ $[(\omega, \tilde{g})]=[(\omega, g)]$. Indeed, since $d_{s}(\omega, g)=0$,

$$
d(Z,(\omega, g), 0,0,0)=(0,0,0, \operatorname{cl}(Z)-\operatorname{cl}(\omega, g), 0)=0 .
$$

To see (ii), assume that $g_{1}, g_{2} \in \mathcal{D}_{\log }^{2 p-1}(X, p)$ are representatives of $\tilde{g}$, i.e. there exists $h \in \mathcal{D}_{\log }^{2 p-2}(X, p)$ such that $d_{\mathscr{D}} h=g_{1}-g_{2}$. Then

$$
\begin{aligned}
d(0,(0, h), 0,0,0) & =\left(0,\left(0, g_{1}-g_{2}\right), 0,0,0\right) \\
& =\left(Z,\left(\omega, g_{1}\right), 0,0,0\right)-\left(Z,\left(\omega, g_{2}\right), 0,0,0\right)
\end{aligned}
$$

and therefore we have $\left[\left(Z,\left(\omega, g_{1}\right), 0,0,0\right)\right]=\left[\left(Z,\left(\omega, g_{2}\right), 0,0,0\right)\right]$.

Finally, to prove (iii), we have to see that, if $Y$ is a codimension $p-1$ subvariety and $f \in k^{*}(Y)$, then

$$
\Phi(\operatorname{div} f, g(f))=0 \in \widehat{\mathrm{CH}}^{p}(X, 0)
$$


i.e. that

$$
[(\operatorname{div} f,(0, g(f)), 0,0,0)]=0,
$$

for any fixed representative $g(f)$ of $\tilde{g}(f)$.

Let $\hat{f}$ be the function of $Y \times \square^{1}$ given by $\left(y,\left(t_{1}: t_{2}\right)\right) \mapsto \frac{t_{1}-t_{2} f(y)}{t_{1}-t_{2}}$. Its divisor defines a codimension $p$ subvariety of $X \times \square^{1}$. Moreover, it intersects properly $X \times(0: 1)$ and $X \times(1: 0)$. Fix $g(\hat{f})$ to be any representative of $\tilde{g}(\hat{f})$. Since $\delta(\tilde{g}(\hat{f}))=\tilde{g}(f)$, there exists $h \in \mathscr{D}_{\log }^{2 p-1}(X \backslash \operatorname{div} f, p)$ with $d_{\mathscr{D}} h=\delta(g(\hat{f}))-g(f)$. Then

$$
d(\operatorname{div} \hat{f},(0, g(\hat{f}),(0, h)), 0,0,0)=(\operatorname{div} f,(0, g(f)), 0,0,0)
$$

as desired.

Lemma 4.10. There are isomorphisms

$$
\begin{aligned}
& \mathrm{CH}^{p}(X) \stackrel{\varphi_{1}}{\longrightarrow} \mathrm{CH}^{p}(X, 0), \\
& \mathrm{CH}^{p-1, p}(X) \stackrel{\varphi_{2}}{\longrightarrow} \mathrm{CH}^{p}(X, 1),
\end{aligned}
$$

making the following diagrams commutative:
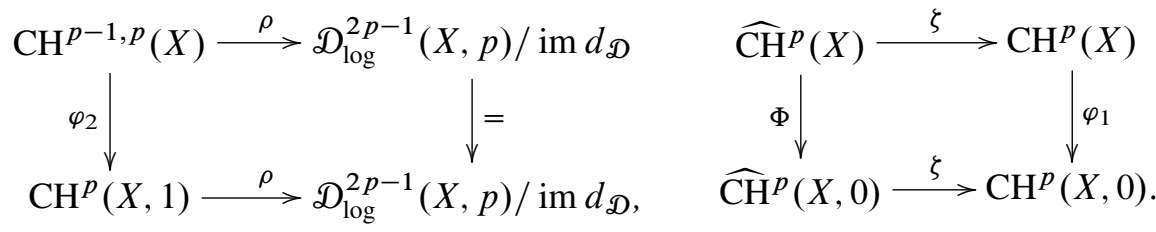

Proof. Both isomorphisms are well known. The morphism $\varphi_{1}$ is the isomorphism between the classical Chow group $\mathrm{CH}^{p}(X)$ and the Bloch Chow group $\mathrm{CH}^{p}(X, 0)$. The diagram is obviously commutative, since $\varphi_{1}([Z])=[Z]$.

The isomorphism $\varphi_{2}$ is defined as follows. Let $f \in \mathrm{CH}^{p-1, p}(X)$. It can be represented by a linear combination $\sum_{i}\left[f_{i}\right]$, where $f_{i} \in k^{*}\left(W_{i}\right), W_{i}$ is a codimension $p-1$ subvariety of $X$ and $\sum \operatorname{div} f_{i}=0$. Let $\Gamma_{f_{i}}$ be the restriction of the graph of $f_{i}$ in $\subset X \times \mathbb{P}^{1}$, to $X \times \square^{1}$. That is, $\Gamma_{f_{i}}$ is the codimension $p$ subvariety of $X \times \square^{1}$ given by

$$
\left\{\left(y, f_{i}(y)\right) \mid y \in W_{i}, f_{i}(y) \neq 1\right\} .
$$

Then $\varphi_{2}(f)$ is represented by the image in

$$
Z^{p}(X, 1) / D Z^{p}(X, 1) \cong Z^{p}(X, 1)_{0}
$$

of $\sum \Gamma_{f_{i}}$, where $D Z^{p}(X, 1)$ are the degenerate elements.

We want to see that $\rho \varphi_{2}=\rho$, i.e., $\rho\left(\sum_{i} \Gamma_{f_{i}}\right)=\rho\left(\sum\left[f_{i}\right]\right)$. See [5] or [8] for more details on the definition of $\rho$ on the right hand side.

Let $f=\sum_{i}\left[f_{i}\right] \in \mathrm{CH}^{p-1, p}(X)$ be as above. For every $i$, we can choose: 
- a rational function $\tilde{f}_{i} \in k^{*}(X)$ whose restriction to $W_{i}$ is $f_{i}$,

- a Green form for $W_{i}, \mathfrak{g}\left(W_{i}\right)=\left(\omega_{i}, g_{i}\right)$.

The form

$$
\mathrm{g}\left(\tilde{f}_{i}\right):=\left(0,-\frac{1}{2} \log \tilde{f}_{i} \overline{\tilde{f}}_{i}\right)
$$

is a Green form for the divisor $\operatorname{div} \tilde{f_{i}}$ on $X$.

Let $\star$ denote the $\star$-product of Green forms as described by Burgos in [5]. Then we write

$$
\left(\omega_{\rho}, \tilde{g}_{\rho}\right)=\sum \mathfrak{g}\left(\tilde{f}_{i}\right) \star \mathfrak{g}\left(W_{i}\right) .
$$

Since the first component of $\mathrm{g}\left(\tilde{f}_{i}\right)$ is zero, we have that $\omega_{\rho}=0$ as well. Moreover, since $\left(0, \tilde{g}_{\rho}\right)$ is a Green form for $\sum_{i} \operatorname{div} \tilde{f}_{i} \cap W_{i}=\sum_{i} \operatorname{div} f_{i}=0$, we can obtain a representative $g_{\rho}$ of $\tilde{g}_{\rho}$ that is a closed smooth form. Then $g_{\rho}$ is a representative of $\rho\left(\sum\left[f_{i}\right]\right)$.

Let us show now that $g_{\rho}$ is a representative of $\rho\left(\varphi_{2}(f)\right)$ as well. By the results of the previous sections, the form $\rho\left(\sum_{i} \Gamma_{f_{i}}\right)$ is obtained as follows. Let $Z \in Z^{p}(X, 1)_{0}$ be a cycle in the normalized group that differs from $\sum \Gamma_{f_{i}}$ by a degenerate element. We consider a representative $\left(\omega_{Z}, g_{Z}\right) \in \tau \mathscr{D}_{Z^{p}}^{2 p}\left(X \times \square_{1}, p\right)_{0}$ of $Z$. Since

$$
\beta=\delta_{1}^{0}\left(\omega_{Z}, g_{Z}\right)-\delta_{1}^{1}\left(\omega_{Z}, g_{Z}\right)
$$

represents the class of $\sum_{i} \operatorname{div} f_{i}=0$, the class of $\beta$ is zero and hence there exists $(\omega, g)$ such that $d_{\mathscr{D}}(\omega, g)=\beta$. Moreover, since $d_{\mathscr{D}} \omega_{Z}=0$ and the complex $\tau \mathscr{D}_{\log }^{*}\left(X \times \square^{1}, p\right)_{0}$ is acyclic (see the proof of Proposition 2.8), there exists $\alpha \in$ $\mathscr{D}_{\log }^{2 p-1}\left(X \times \square^{1}, p\right)_{0}$ such that $d_{\mathscr{D}}(\alpha)=\omega_{Z}$. Then $\rho\left(\sum_{i} \Gamma_{f_{i}}\right)$ is represented by $\omega+\delta(\alpha)$.

Therefore, we start by constructing the cycle $Z$ and suitable forms $\left(\omega_{Z}, g_{Z}\right)$ representing the class of $Z$. Consider the rational function $h_{i} \in k^{*}\left(X \times \square^{1}\right)$ given by

$$
\left(y,\left(t_{1}: t_{2}\right)\right) \mapsto \frac{t_{1}-t_{2} \tilde{f}_{i}(y)}{t_{1}-t_{2}} .
$$

If we write $\operatorname{div} f_{i}=\left(\operatorname{div} f_{i}\right)^{0}-\left(\operatorname{div} f_{i}\right)^{\infty}$ where $\left(\operatorname{div} f_{i}\right)^{0}$ is the divisor of zeroes and $\left(\operatorname{div} f_{i}\right)^{\infty}$ is the divisor of poles, the intersection of the divisor of $h_{i}$ with $W_{i}$, $\operatorname{div} h_{i} \cap W_{i}$, is exactly $\Gamma_{f_{i}}-\left(\operatorname{div} f_{i}\right)^{\infty}$. Observe that $\left(\operatorname{div} f_{i}\right)^{\infty}$ is a codimension $p$ degenerate cycle. Moreover $\operatorname{div} h_{i} \cap W_{i}$ belongs to $Z^{p}(X, 1)_{0}$. Hence

$$
Z=\sum \operatorname{div} h_{i} \cap W_{i}
$$

is the cycle we need. Let $\mathrm{g}\left(h_{i}\right)=\left(0,-\frac{1}{2} \log h_{i} \bar{h}_{i}\right)$ be the canonical Green form for $\operatorname{div} h_{i}$. Then, as above, a Green form for $Z$ is given by

$$
\sum \mathfrak{g}\left(h_{i}\right) \star \mathfrak{g}\left(W_{i}\right)=\left(0, \tilde{g}_{Z}\right) .
$$


Now, observe that

$$
\delta\left(0, \tilde{g}_{Z}\right)=\sum_{i} \delta_{1}^{0}\left(\mathfrak{g}\left(h_{i}\right)\right) \star \mathfrak{g}\left(W_{i}\right)=\sum_{i} \mathfrak{g}\left(\tilde{f}_{i}\right) \star \mathfrak{g}\left(W_{i}\right)=\left(0, \tilde{g}_{\rho}\right) .
$$

Since we can assume that $g_{\rho}$ is a smooth representative of $\tilde{g}_{\rho}$, we have that $d_{s}\left(g_{\rho}, 0\right)=$ $\left(0, g_{\rho}\right)$, and hence by the above description of $\rho$ we see that

$$
\rho\left(\sum_{i} \Gamma_{f_{i}}\right)=g_{\rho}
$$

This finishes the proof of the lemma.

Lemma 4.11. The following diagram is commutative:

$$
\mathscr{D}_{\log }^{2 p-1}(X, p) / \mathrm{im} d_{\mathscr{D}} \stackrel{\mathrm{a}}{\underset{\mathrm{a}}{\longrightarrow} \widehat{\mathrm{CH}}^{p}(X)} \widehat{\mathrm{CH}}^{p}(X, 0) .
$$

Proof. Let $\tilde{\alpha} \in \mathscr{D}_{\log }^{2 p-1}(X, p) / \operatorname{im} d_{\mathscr{D}}$. Then the lemma follows from the equality

$$
d(0,(\alpha, 0), 0,0,0)=\left(0,\left(d_{\mathscr{D}} \alpha, \alpha\right), 0,0,0\right)+(0,0,0,0, \alpha)
$$

in $\widehat{\mathrm{CH}}^{p}(X, 0)$.

This finishes the proof of Theorem 4.8 .

\subsection{Functoriality of the higher arithmetic Chow groups}

Proposition 4.12 (Pull-back). Let $f: X \rightarrow Y$ be a morphism between two arithmetic varieties. Then, for all $p \geq 0$, there exists a chain complex, $\widehat{Z}_{f}^{p}(Y, *)_{0}$ such that:

(i) There is a quasi-isomorphism

$$
\hat{Z}_{f}^{p}(Y, *)_{0} \stackrel{\sim}{\rightarrow} \hat{Z}^{p}(Y, *)_{0} .
$$

(ii) There is a pull-back morphism

$$
f^{*}: \widehat{Z}_{f}^{p}(Y, *)_{0} \rightarrow \widehat{Z}^{p}(X, *)_{0},
$$

inducing a pull-back morphism of higher arithmetic Chow groups

$$
\widehat{\mathrm{CH}}^{p}(Y, n) \stackrel{f^{*}}{\longrightarrow} \widehat{\mathrm{CH}}^{p}(X, n),
$$

for every $p, n \geq 0$. 
(iii) The pull-back is compatible with the morphisms a and $\zeta$. That is, there are commutative diagrams

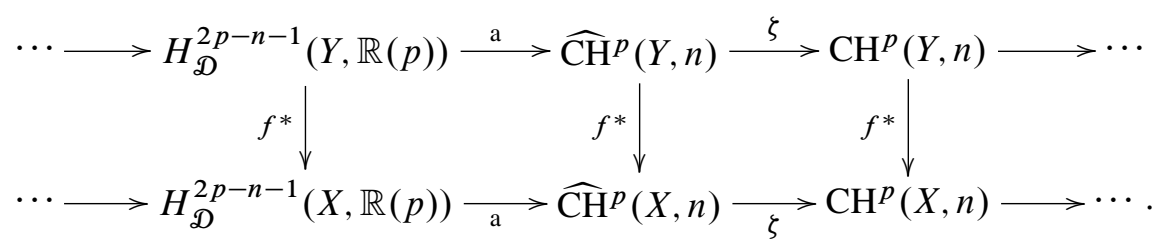

Proof. Recall that there are inclusions of complexes

$$
\begin{aligned}
Z_{f}^{p}(Y, *)_{0} & \subseteq Z^{p}(Y, *)_{0}, \\
\mathscr{H}_{f}^{p}(Y, *)_{0} & \subseteq \mathscr{H}^{p}(Y, *)_{0}, \\
\mathscr{D}_{\mathbb{A}, Z_{f}^{p}}^{*}(Y, p)_{0} & \subseteq \mathscr{D}_{\mathbb{A}, Z^{p}}^{*}(Y, p)_{0},
\end{aligned}
$$

which are quasi-isomorphisms. The pull-back by $f$ is defined for any $\alpha$ in $Z_{f}^{p}(Y, *)_{0}$, in $\mathscr{H}_{f}^{p}(Y, *)_{0}$ or in $\mathscr{D}_{\mathbb{A}, \mathcal{Z}_{f}^{p}}^{*}(Y, p)_{0}$. Moreover, by construction, there is a commutative diagram

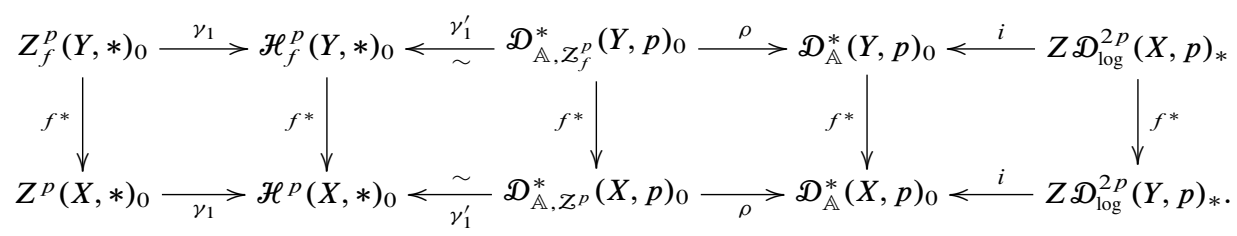

Let $\hat{Z}_{f}^{p}(Y, *)_{0}$ denote the simple associated to the first row diagram. Then there is a pull-back morphism

$$
f^{*}: \widehat{Z}_{f}^{p}(Y, *)_{0} \rightarrow \widehat{Z}^{p}(X, *)_{0} .
$$

Moreover, as noticed in $§ 1.3$, the natural map

$$
\hat{Z}_{f}^{p}(Y, *)_{0} \rightarrow \hat{Z}^{p}(Y, *)_{0}
$$

is a quasi-isomorphism. Therefore, (i) and (ii) are proved. Statement (iii) follows from the construction.

Remark 4.14. If the map is flat, then the pull-back is already defined at the level of the chain complexes $\widehat{Z}^{p}(Y, *)_{0}$ and $\hat{Z}^{p}(X, *)_{0}$.

Proposition 4.15 (Functoriality of pull-back). Let $f: Y$ and $g: Y \rightarrow Z$ be two morphisms of arithmetic varieties. Then

$$
f^{*} \circ g^{*}=(g \circ f)^{*}: \widehat{\mathrm{CH}}^{p}(Z, n) \rightarrow \widehat{\mathrm{CH}}^{p}(X, n) .
$$


Proof. Let $\hat{Z}_{g f \cup g}^{p}(Z, n)_{0}$ be the subgroup of $\hat{Z}^{p}(Z, n)_{0}$ obtained considering, at each of the complexes of the diagram $\widehat{Z}^{p}(Z, *)_{0}$, the subvarieties $W$ of $Z \times \square^{n}$ intersecting properly the faces of $\square^{n}$ and such that

- $X \times W \times \square^{n}$ intersects properly the graph of $g \circ f$,

- $Y \times W \times \square^{n}$ intersects properly the graph of $g$.

That is,

$$
\hat{Z}_{g f \cup g}^{p}(Z, n)_{0}=\hat{Z}_{g f}^{p}(Z, n)_{0} \cap \hat{Z}_{g}^{p}(Z, n)_{0} .
$$

Then the proposition follows from the commutative diagram

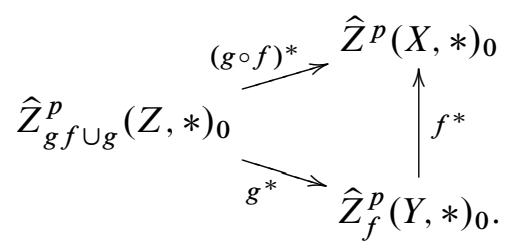

Corollary 4.16 (Homotopy invariance). Let $\pi: X \times \mathbb{A}^{m} \rightarrow X$ be the projection on $X$. Then the pull-back map

$$
\pi^{*}: \widehat{\mathrm{CH}}^{p}(X, n) \rightarrow \widehat{\mathrm{CH}}^{p}\left(X \times \mathbb{A}^{m}, n\right)
$$

is an isomorphism for all $n \geq 1$.

Proof. It follows from the five lemma in the diagram (4.13), using the fact that both the higher Chow groups and the Deligne-Beilinson cohomology groups are homotopy invariant.

\section{Product structure}

Let $X, Y$ be arithmetic varieties over an arithmetic field $K$. In this section, we define an external product, $\widehat{\mathrm{CH}}^{*}(X, *) \otimes \widehat{\mathrm{CH}}^{*}(Y, *) \rightarrow \widehat{\mathrm{CH}}^{*}(X \times Y$,*), and an internal product $\widehat{\mathrm{CH}}^{*}(X, *) \otimes \widehat{\mathrm{CH}}^{*}(X, *) \rightarrow \widehat{\mathrm{CH}}^{*}(X, *)$, for the higher arithmetic Chow groups. The internal product endows $\widehat{\mathrm{CH}}^{*}(X, *)$ with a ring structure. It will be shown that this product is commutative and associative. There are two main technical difficulties. The first one is that we are representing a cohomology class with support in a cycle by a pair of forms, the first one smooth on the whole variety and the second one with singularities along the cycle. The product of two singular forms has singularities along the union of the singular locus. Therefore, in order to define a cohomology class with support on the intersection of two cycles we need a little bit of homological algebra. To this end we adapt the technique used in [5]. The second difficulty is that the external product in higher Chow groups is not graded 
commutative at the level of complexes, but only graded commutative up to homotopy. To have explicit homotopies we will adapt the techniques of [19].

Recall that the higher arithmetic Chow groups are the homology groups of the simple complex associated to a diagram of complexes. Therefore, in order to define a product, we use the general procedure developed by Beilinson, as recalled in $\$ 1.4$. To this end, we need to define a product for each of the complexes in the diagram $\widehat{\mathcal{Z}}^{p}(X, *)_{0}(4.1)$, commuting with the morphisms $\gamma_{1}, \gamma_{1}^{\prime}, \rho$ and $i$. The pattern for the external product construction is analogous to the pattern followed to define the external product for the cubical higher Chow groups, described in $\$ 2.3$.

For the complex $Z^{p}(X, *)_{0}$ we already have an external product recalled in $\S 2.3$. Since the complex $\mathscr{H}^{p}(X, *)_{0}$ is isomorphic to $Z_{\mathbb{R}}^{p}\left(X_{\mathbb{R}}, *\right)_{0}$, the external product on the complex $\mathscr{H}^{*}(X, *)_{0}$ can be defined by means of this isomorphism. We will now construct the product for the remaining complexes.

5.1. Product structure on the complexes $\mathscr{D}_{\mathbb{A}}^{*}(X, p)$ and $Z \mathscr{D}_{\log }^{2 p}(X, p)_{*}$. We start by defining a product structure on $\mathscr{D}_{\mathbb{A}}^{*}(X, p)$. Let

$$
X \times Y \times \square^{n} \times \square^{m} \stackrel{p_{13}}{\longrightarrow} X \times \square^{n}, \quad X \times Y \times \square^{n} \times \square^{m} \stackrel{p_{24}}{\longrightarrow} Y \times \square^{m}
$$

be the projections indicated by the subindices. For every $\omega_{1} \in \tau \mathcal{D}_{\log }^{r}\left(X \times \square^{n}, p\right)$ and $\omega_{2} \in \tau \mathscr{D}_{\log }^{s}\left(Y \times \square^{m}, q\right)$, we define

$$
\omega_{1} \bullet \mathbb{A} \omega_{2}:=(-1)^{n s} p_{13}^{*} \omega_{1} \bullet p_{24}^{*} \omega_{2} \in \tau \mathcal{D}_{\log }^{r+s}\left(X \times Y \times \square^{n+m}, p+q\right),
$$

where $\bullet$ in the right hand side is the product in the Deligne complex (see $§ 1.8$ ).

This gives a map

$$
\begin{aligned}
\mathcal{D}_{\mathbb{A}}^{r_{1}}(X, p) \otimes \mathcal{D}_{\mathbb{A}}^{r_{2}}(Y, q) \stackrel{\bullet \mathbb{A}}{\longrightarrow} \mathcal{D}_{\mathbb{A}}^{r_{1}+r_{2}}(X \times Y, p+q), \\
\left(\omega_{1}, \omega_{2}\right) \stackrel{\longmapsto}{\longmapsto} \omega_{1} \bullet \mathbb{A} \omega_{2} .
\end{aligned}
$$

Lemma 5.1. The map $\bullet_{\mathbb{A}}$ satisfies the Leibniz rule. Therefore, there is a cochain morphism

$$
s\left(\mathcal{D}_{\mathbb{A}}^{*}(X, p) \otimes \mathcal{D}_{\mathbb{A}}^{*}(Y, q)\right) \stackrel{\bullet}{\longrightarrow} D_{\mathbb{A}}^{*}(X \times Y, p+q) .
$$

Proof. Let $\omega_{1} \in \tau \mathscr{D}_{\log }^{r}(X, n)$ and $\omega_{2} \in \tau \mathscr{D}_{\log }^{s}(Y, m)$. By definition of $\delta$, the following equality holds

$$
\delta\left(p_{13}^{*} \omega_{1} \bullet p_{24}^{*} \omega_{2}\right)=p_{13}^{*}\left(\delta \omega_{1}\right) \bullet p_{24}^{*} \omega_{2}+(-1)^{n} p_{13}^{*} \omega_{1} \bullet p_{24}^{*}\left(\delta \omega_{2}\right)
$$


Then

$$
\begin{aligned}
d_{s}\left(\omega_{1} \bullet \mathbb{A} \omega_{2}\right)= & (-1)^{n s} d_{s}\left(p_{13}^{*} \omega_{1} \bullet p_{24}^{*} \omega_{2}\right) \\
= & (-1)^{n s} d_{\mathscr{D}}\left(p_{13}^{*} \omega_{1} \bullet p_{24}^{*} \omega_{2}\right)+(-1)^{r+s+n s} \delta\left(p_{13}^{*} \omega_{1} \bullet p_{24}^{*} \omega_{2}\right) \\
= & (-1)^{n s} d_{\mathcal{D}}\left(p_{13}^{*} \omega_{1}\right) \bullet p_{24}^{*} \omega_{2}+(-1)^{r+n s} p_{13}^{*} \omega_{1} \bullet d_{\mathscr{D}}\left(p_{24}^{*} \omega_{2}\right) \\
& +(-1)^{r+s+n s} p_{13}^{*}\left(\delta \omega_{1}\right) \bullet p_{24}^{*} \omega_{2} \\
& \quad+(-1)^{r+s+n+n s} p_{13}^{*} \omega_{1} \bullet p_{24}^{*}\left(\delta \omega_{2}\right) \\
= & d_{\mathscr{D}} \omega_{1} \bullet \mathbb{A} \omega_{2}+(-1)^{r+n} \omega_{1} \bullet \mathbb{A} d_{\mathscr{D}}\left(\omega_{2}\right) \\
& +(-1)^{r} \delta \omega_{1} \bullet \mathbb{A} \omega_{2}+(-1)^{r+n+s} \omega_{1} \bullet \mathbb{A} \delta\left(\omega_{2}\right) \\
= & d_{S}\left(\omega_{1}\right) \bullet \mathbb{A} \omega_{2}+(-1)^{r+n} \omega_{1} \bullet \mathbb{A} d_{s}\left(\omega_{2}\right),
\end{aligned}
$$

as desired.

Definition 5.2. Let $\tau \mathscr{D}_{\log }^{*}\left(X \times Y \times \square^{*} \times \square^{*}, p\right)_{0}$ be the 3 -iterated cochain complex whose $(r,-n,-m)$-th graded piece is the group $\tau \mathcal{D}_{\log }^{r}\left(X \times Y \times \square^{n} \times \square^{m}, p\right)_{0}$ and whose differentials are $\left(d_{\mathscr{D}}, \delta, \delta\right)$. Let

$$
\mathcal{D}_{\mathbb{A} \times \mathbb{A}}^{*}(X \times Y, p)_{0}:=s\left(\tau \mathcal{D}_{\log }^{*}\left(X \times Y \times \square^{*} \times \square^{*}, p\right)_{0}\right)
$$

be the associated simple complex.

Remark 5.4. Observe that there is a cochain morphism

$$
\mathscr{D}_{\mathbb{A} \times \mathbb{A}}^{*}(X \times Y, p)_{0} \stackrel{\kappa}{\rightarrow} \mathscr{D}_{\mathbb{A}}^{*}(X \times Y, p)_{0}
$$

sending $\alpha \in \tau \mathscr{D}_{\log }^{r}\left(X \times Y \times \square^{n} \times \square^{m}, p\right)$ to $\alpha \in \tau \mathscr{D}_{\log }^{r}\left(X \times Y \times \square^{n+m}, p\right)$ under the identification

$$
\begin{aligned}
\square^{n+m} & \stackrel{\cong}{\longrightarrow} \square^{n} \times \square^{m}, \\
\left(x_{1}, \ldots, x_{n+m}\right) & \longmapsto\left(\left(x_{1}, \ldots, x_{n}\right),\left(x_{n+1}, \ldots, x_{n+m}\right)\right) .
\end{aligned}
$$

Moreover, the product $\bullet_{A}$ that we have defined previously, factors through the morphism $\kappa$ and a product, also denoted by $\bullet A$,

$$
\mathscr{D}_{\mathbb{A}}^{*}(X, p) \otimes \mathscr{D}_{\mathbb{A}}^{*}(Y, q) \stackrel{\bullet \mathbb{A}}{\longrightarrow} D_{\mathbb{A} \times \mathbb{A}}^{*}(X \times Y, p+q) .
$$

In order to define the product on the complex $Z \mathcal{D}_{\log }^{2 p}(X, p)_{*}$, recall that we have an isomorphism (see [5])

$$
Z \mathscr{D}_{\log }^{2 p}(X, p) \cong Z E_{\log , \mathbb{R}}^{p, p}(X)(p)
$$


and that the restriction of the product $\bullet$ to this subspace is given by the product $\wedge$.

The inclusion $i$ is compatible with the product $\bullet_{\mathbb{A}}$ and the product $\wedge$. That is, consider the projections $p_{X}: X \times Y \rightarrow X$ and $p_{Y}: X \times Y \rightarrow Y$. Then, if $\alpha \in Z E_{\log , \mathbb{R}}^{p, p}(X)(p)$ and $\beta \in Z E_{\log , \mathbb{R}}^{q, q}(Y)(q)$, we put

$$
\alpha \wedge \beta=p_{X}^{*}(\alpha) \wedge p_{Y}^{*}(\beta) \in Z E_{\log , \mathbb{R}}^{p+q, p+q}(X \times Y)(p+q) .
$$

We have a commutative diagram

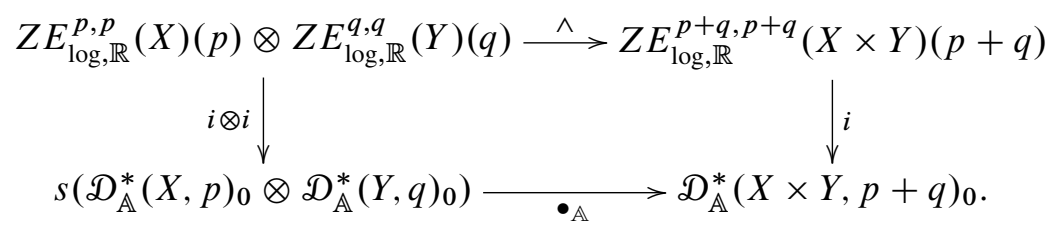

5.2. Product structure on the complex $\mathscr{D}_{\mathbb{A}, \mathfrak{Z}^{p} \boldsymbol{p}}^{*}(X, p)$. We define here a product on the complex $\mathscr{D}_{\mathbb{A}, \mathcal{Z}^{p}}^{*}(X, p)$. It will be compatible with the product on $\mathscr{D}_{\mathbb{A}}^{*}(X, p)$, under the morphism $\rho$, and with the product on $\mathscr{H}^{p}(X, *)_{0}$ under $\gamma_{1}^{\prime}$.

Let $X, Y$ be two real varieties. For every $p$, let $\mathcal{Z}_{X, n}^{p}$ be the subset of codimension $p$ subvarieties of $X \times \square^{n}$ intersecting properly the faces of $\square^{n}$. Let

$$
\mathcal{Z}_{X, Y, n, m}^{p, q} \subseteq \mathcal{Z}_{X \times Y, n+m}^{p+q}
$$

be the subset of the set of codimension $p+q$ subvarieties of $X \times Y \times \square^{n+m}$, intersecting properly the faces of $\square^{n+m}$, which are obtained as the cartesian product $Z \times W$ with $Z \in \mathcal{Z}_{X, n}^{p}$ and $W \in \mathcal{Z}_{Y, m}^{q}$.

For shorthand, we make the following identifications:

$$
\begin{gathered}
Z_{Y, m}^{q}=\left\{X \times Z \mid Z \in \mathcal{Z}_{Y, m}^{q}\right\} \subseteq Z_{X \times Y, n+m}^{q}, \\
Z_{X, n}^{p}=\left\{W \times Y \mid W \in Z_{X, m}^{p}\right\} \subseteq Z_{X \times Y, n+m}^{p} .
\end{gathered}
$$

To ease the notation, we write temporarily

$$
\square_{X, Y}^{n, m}:=X \times Y \times \square^{n} \times \square^{m} .
$$

For every $n, m, p, q$, let $j_{X, Y}^{p, q}(n, m)$ be the morphism

$$
\begin{aligned}
\mathscr{D}_{\log }^{*}\left(\square_{X, Y}^{n, m} \backslash \mathcal{Z}_{X, n}^{p}, p+q\right) \oplus \mathcal{D}_{\log }^{*}\left(\square_{X, Y}^{n, m} \backslash \mathcal{Z}_{Y, m}^{q}, p+q\right) \\
\stackrel{j_{X, Y}^{p, q}(n, m)}{\longrightarrow} \mathcal{D}_{\log }^{*}\left(\square_{X, Y}^{n, m} \backslash \mathcal{Z}_{X, n}^{p} \cup \mathcal{Z}_{Y, m}^{q}, p+q\right)
\end{aligned}
$$

induced on the limit complexes by the morphism $j$ in Lemma 1.29. 
Lemma 5.5. There is a short exact sequence

$$
\begin{aligned}
0 \longrightarrow & \mathbb{D}_{\log }^{*}\left(\square_{X, Y}^{n, m} \backslash \mathcal{Z}_{X, Y, n, m}^{p, q}, p+q\right) \\
\longrightarrow & \mathbb{D}_{\log }^{*}\left(\square_{X, Y}^{n, m} \backslash \mathcal{Z}_{X, n}^{p}, p+q\right) \oplus \mathcal{D}_{\log }^{*}\left(\square_{X, Y}^{n, m} \backslash \mathcal{Z}_{Y, m}^{q}, p+q\right) \\
& \stackrel{j_{X, Y}^{p, q}(n, m)}{\longrightarrow} \mathbb{D}_{\log }^{*}\left(\square_{X, Y}^{n, m} \backslash \mathcal{Z}_{X, n}^{p} \cup \mathcal{Z}_{Y, m}^{q}, p+q\right) \rightarrow 0 .
\end{aligned}
$$

Proof. This follows from Lemma 1.29.

By the quasi-isomorphism between the simple complex and the kernel of an epimorphism (see (1.2)), for every $n, m$, there is a quasi-isomorphism

$$
\begin{aligned}
\mathcal{D}_{\log }^{*}\left(\square_{X, Y}^{n, m} \backslash \mathcal{Z}_{X, Y, n, m}^{p, q}, p+q\right) & \stackrel{\sim}{\longrightarrow} s\left(-j_{X, Y}^{p, q}(n, m)\right)^{*} \\
\omega & \longmapsto(\omega, \omega, 0) .
\end{aligned}
$$

It induces a quasi-isomorphism

$$
\mathcal{D}_{\log , Z_{X, Y, n, m}^{p, q}}^{*}\left(\square_{X, Y}^{n, m}, p+q\right) \stackrel{\sim}{\rightarrow} s\left(\mathcal{D}_{\log }^{*}\left(\square_{X, Y}^{n, m}, p+q\right)^{*} \stackrel{i_{X, Y}^{p, q}(n, m)}{\longrightarrow} s\left(-j_{X, Y}^{p, q}(n, m)\right)\right)^{*},
$$

where $i_{X, Y}^{p, q}(n, m)$ is defined by

$$
\begin{aligned}
& \mathbb{D}_{\log }^{*}\left(\square_{X, Y}^{n, m}, p+q\right) \stackrel{i_{X, Y}^{p, q}(n, m)}{\longrightarrow} s\left(-j_{X, Y}^{p, q}(n, m)\right)^{*}, \\
& \omega \quad \longmapsto \quad(\omega, \omega, 0) .
\end{aligned}
$$

Remark 5.7. Observe that there is an induced bicubical cochain complex structure on $s\left(i_{X, Y}^{p, q}(\cdot, \cdot)\right)^{*}$. For every $r$, let $s\left(i_{X, Y}^{p, q}(*, *)\right)_{0}^{r}$ denote the 2 -iterated complex obtained by taking the normalized complex functor to both cubical structures. Consider the 3 -iterated complex $s\left(i_{X, Y}^{p, q}(*, *)\right)_{0}^{*}$ whose piece of degree $(r,-n,-m)$ is the group $\tau_{r \leq 2 p+2 q} s\left(i_{X, Y}^{p, q}(n, m)\right)_{0}^{r}$, and whose differential is $\left(d_{s}, \delta, \delta\right)$. Denote by $s\left(i_{X, Y}^{p, q}\right)_{0}^{*}$ the associated simple complex. Observe that the differential of $\alpha=\left(\alpha_{0},\left(\alpha_{1}, \alpha_{2}\right), \alpha_{3}\right) \in$ $s\left(i_{X, Y}^{p, q}\right)_{0}^{r}$ is given by

$$
d_{S}^{\prime}\left(\alpha_{0},\left(\alpha_{1}, \alpha_{2}\right), \alpha_{3}\right)=\left(d_{\mathscr{D}} \alpha_{0},\left(\alpha_{0}-d_{\mathscr{D}} \alpha_{1}, \alpha_{0}-d_{\mathscr{D}} \alpha_{2}\right),-\alpha_{1}+\alpha_{2}+d_{\mathscr{D}} \alpha_{3}\right) .
$$

Definition 5.8. Let $\bullet_{\mathbb{A}}$ be the map

$$
\mathscr{D}_{\log , Z^{p}}^{r}\left(X \times \square^{n}, p\right)_{0} \otimes \mathcal{D}_{\log , Z^{q}}^{s}\left(Y \times \square^{m}, q\right)_{0} \stackrel{\bullet}{\longrightarrow} s\left(i_{X, Y}^{p, q}(n, m)\right)_{0}^{r+s}
$$

defined by sending $(\omega, g) \otimes\left(\omega^{\prime}, g^{\prime}\right)$ to

$$
(-1)^{n s}\left(\omega \bullet \omega^{\prime},\left(g \bullet \omega^{\prime},(-1)^{r} \omega \bullet g^{\prime}\right),(-1)^{r-1} g \bullet g^{\prime}\right) .
$$


Lemma 5.9. The map $\bullet \mathbb{A}$ defines a pairing of complexes

$$
s\left(\mathscr{D}_{\mathbb{A}, Z^{p}}^{*}(X, p)_{0} \otimes \mathbb{D}_{\mathbb{A}, Z^{q}}^{*}(Y, q)_{0}\right) \stackrel{\bullet}{\longrightarrow} s\left(i_{X, Y}^{p, q}\right)_{0}^{*} .
$$

Proof. Let $(\omega, g) \in \mathscr{D}_{\log , Z^{p}}^{r}\left(X \times \square^{n}, p\right)_{0}$ and $\left(\omega^{\prime}, g^{\prime}\right) \in \mathscr{D}_{\log , Z^{q}}^{s}\left(Y \times \square^{m}, q\right)_{0}$. Then we have to see that

$$
d_{s}^{\prime}\left((\omega, g) \bullet_{\mathbb{A}}\left(\omega^{\prime}, g^{\prime}\right)\right)=d_{s}^{\prime}(\omega, g) \bullet_{\mathbb{A}}\left(\omega^{\prime}, g^{\prime}\right)+(-1)^{r-n}(\omega, g) \bullet_{\mathbb{A}} d_{s}^{\prime}\left(\omega^{\prime}, g^{\prime}\right) .
$$

That is, we have to show that the following two equalities hold:

$$
\begin{aligned}
d_{s}((\omega, g) \bullet \mathbb{A} & \left.\left(\omega^{\prime}, g^{\prime}\right)\right)=d_{s}(\omega, g) \bullet_{\mathbb{A}}\left(\omega^{\prime}, g^{\prime}\right)+(-1)^{r-n}(\omega, g) \bullet_{\mathbb{A}} d_{s}\left(\omega^{\prime}, g^{\prime}\right), \\
\delta\left((\omega, g) \bullet \mathbb{A}\left(\omega^{\prime}, g^{\prime}\right)\right) & =(-1)^{s} \delta(\omega, g) \bullet_{\mathbb{A}}\left(\omega^{\prime}, g^{\prime}\right)+(-1)^{n}(\omega, g) \bullet_{\mathbb{A}} \delta\left(\omega^{\prime}, g^{\prime}\right) .
\end{aligned}
$$

The proof of the second equality is analogous to the proof of Lemma 5.1. The first equality is a direct computation.

We define a complex $\mathscr{D}_{\mathbb{A} \times \mathbb{A}, Z_{X, Y}^{*, q}}^{*}(X \times Y, p+q)_{0}$ that is analogous to the complex $\mathscr{D}_{\mathbb{A} \times \mathbb{A}}^{*}(X, p)_{0}$ of Definition 5.2.

Definition 5.10. Let $\mathscr{D}_{\mathbb{A} \times \mathbb{A}, Z_{X, Y}^{*, q}}^{*}(X \times Y, p+q)_{0}$ be the simple complex associated to the 3-iterated complex whose $(r,-n,-m)$ graded piece is

$$
\tau \mathscr{D}_{\log , \mathcal{Z}_{X, Y, n, m}^{p, q}}^{r}\left(X \times Y \times \square^{n} \times \square^{m}, p+q\right)_{0} .
$$

As in Remark 5.4, we will denote by $\kappa$ the morphisms obtained by identifying $\square^{n} \times \square^{m}$ with $\square^{n+m}$.

$$
\mathscr{D}_{\mathbb{A} \times \mathbb{A}, Z^{p+q}}^{*}(X \times Y, p+q)_{0} \stackrel{\kappa}{\longrightarrow} \mathscr{D}_{\mathbb{A}, Z^{p+q}}^{*}(X \times Y, p+q)_{0} .
$$

We will denote by $\rho$ the morphisms obtained by forgetting the support:

$$
\begin{array}{r}
\mathscr{D}_{\mathbb{A} \times \mathbb{A}, Z_{X, Y}^{p, q}}^{*}(X \times Y, p+q)_{0} \stackrel{\rho}{\longrightarrow} D_{\mathbb{A} \times \mathbb{A}}^{*}(X \times Y, p)_{0}, \\
s\left(i_{X, Y}^{p, q}\right)_{0}^{*} \stackrel{\rho}{\longrightarrow} D_{\mathbb{A} \times \mathbb{A}}^{*}(X \times Y, p)_{0} .
\end{array}
$$

There are also natural morphisms, whose definitions are obvious,

$$
\begin{aligned}
& \mathcal{D}_{\mathbb{A} \times \mathbb{A}, Z_{X, Y}^{p, q}}^{*}(X \times Y, p+q)_{0} \longrightarrow \mathcal{D}_{\mathbb{A} \times \mathbb{A}, Z^{p+q}}^{*}(X \times Y, p+q)_{0}, \\
& \mathcal{D}_{\mathbb{A} \times \mathbb{A}, Z_{X, Y}^{p, q}}^{*}(X \times Y, p+q)_{0} \longrightarrow s\left(i_{X, Y}^{p, q}\right)_{0}^{*} .
\end{aligned}
$$

Lemma 5.11. The natural map

$$
\mathscr{D}_{\mathbb{A} \times \mathbb{A}, Z_{X, Y}^{p, q}}^{*}(X \times Y, p+q)_{0} \rightarrow s\left(i_{X, Y}^{p, q}\right)_{0}^{*}
$$

is a quasi-isomorphism. Moreover, it commutes with $\rho$. 
Proof. It follows from the quasi-isomorphism (5.6).

The external product on $\mathscr{D}_{\mathbb{A}, Z^{*}}^{*}(\cdot, *)_{0}$ is given, in the derived category of complexes, by

$$
\begin{aligned}
& \mathscr{D}_{\mathbb{A}, Z_{X}^{p}}^{r}(X, p)_{0} \otimes \mathcal{D}_{\mathbb{A}, Z_{Y}^{q}}^{s}(Y, q)_{0} \stackrel{\bullet_{\mathbb{A}}}{\longrightarrow} s\left(i_{X, Y}^{p, q}\right)_{0}^{r+s} \\
& \sim \uparrow \\
& \mathcal{D}_{\mathbb{A} \times \mathbb{A}, \mathcal{Z}_{X, Y}^{p, q}}^{r+s}(X \times Y, p+q)_{0} \stackrel{\kappa}{\longrightarrow} \mathcal{D}_{\mathbb{A}, \mathcal{Z}_{X \times Y}^{p+q}}^{r+s}(X \times Y, p+q)_{0} .
\end{aligned}
$$

The fact that to define the product in this complex we need to invert a quasi-isomorphism is the main reason of the complexity of the definition of the product on the higher arithmetic Chow groups.

By definition, it is clear that this morphism commutes with the morphism defined on the complex $\mathscr{D}_{\mathbb{A}}^{*}(X, p)$. It remains to be seen that the product on $\mathscr{D}_{\mathbb{A}, \mathcal{Z}^{p}}^{2 p-n}(X, p)_{0}$ is compatible with the product on $\mathscr{H}^{p}(X, n)_{0}$, under the quasi-isomorphism $\gamma_{1}^{\prime}$.

Let $\omega \in s\left(i_{X \times Y}^{p, q}\right)_{0}^{2 p+2 q-l}$ and let

$$
\left(\omega_{l}^{0}, \ldots, \omega_{l}^{l}\right) \in \bigoplus_{j=0}^{l} \tau_{\leq 2 p+2 q} s\left(i_{X, Y}^{p, q}(j, l-j)\right)_{0}^{2 p+2 q}
$$

be the components of $\omega$ corresponding to the degree $(2 p+2 q,-j, j-l)$. These are the components that have maximal degree as differential forms and, by the definition of the truncated complex they satisfy $d_{s} \omega_{l}^{j}=0$. Thus, the form $\omega_{l}^{j}$ defines a cohomology class $\left[\omega_{l}^{j}\right]$ in the complex $s\left(i_{X, Y}^{p, q}(j, l-j)\right)_{0}^{*}$. Since there is a quasiisomorphism

$$
\mathcal{D}_{\log , \mathcal{L}_{X, Y}^{p, q}}^{*}\left(X \times Y \times \square^{l}, p+q\right)_{0} \stackrel{\sim}{\rightarrow} s\left(i_{X, Y}^{p, q}(j, l-j)\right)_{0}^{*},
$$

we obtain a cohomology class in $H^{*}\left(\mathcal{D}_{\log , \mathcal{Z}_{X, Y}^{p, q}}\left(X \times Y \times \square^{l}, p+q\right)_{0}\right)$. Hence, a cohomology class $\left[\omega_{l}^{j}\right] \in \mathscr{H}^{p+q}(X \times Y, l)_{0}$. This procedure defines a chain morphism, denoted by $\gamma_{1}^{\prime}$,

$$
\begin{aligned}
s\left(i_{X, Y}^{p, q}\right)_{0}^{2 p+2 q-l} & \stackrel{\gamma_{1}^{\prime}}{\longrightarrow} \mathscr{H}^{p+q}(X \times Y, l)_{0}, \\
\omega & \longmapsto \sum_{j}\left[\omega_{l}^{j}\right] .
\end{aligned}
$$

By composition, we can define a morphism, also denoted by $\gamma_{1}^{\prime}$,

$$
\mathscr{D}_{\mathbb{A} \times A, \mathcal{Z}_{X, Y}^{p, q}}^{*}(X \times Y, p+q)_{0} \stackrel{\gamma_{1}^{\prime}}{\longrightarrow} \mathscr{H}^{p+q}(X \times Y, *)_{0} .
$$


Moreover there is a commutative diagram

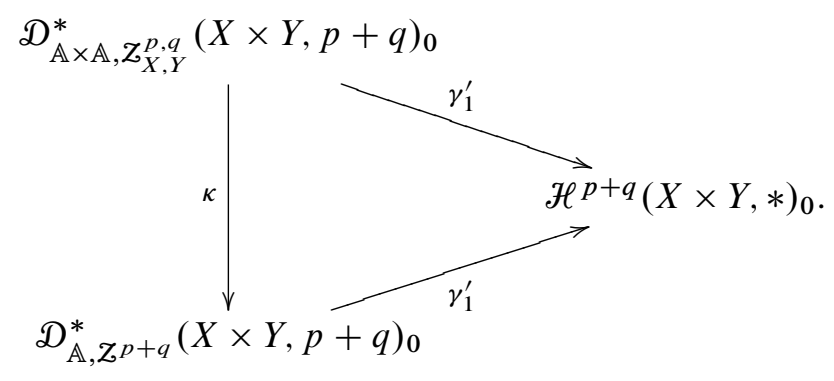

Proposition 5.13. Let $Z \in \mathcal{Z}_{X, n}^{p}$ and $T \in \mathcal{Z}_{Y, m}^{q}$. Let $\left[\left(\omega_{Z}, g_{Z}\right)\right] \in \mathscr{H}^{p}(X, n)_{0}$ represent the class of a cycle $z \in Z^{p}(X, n)_{0}$ with support on $Z$ and let $\left[\left(\omega_{T}, g_{T}\right)\right] \in \mathscr{H}^{q}(Y, m)_{0}$ represent the class of a cycle $t \in Z^{q}(Y, m)_{0}$ with support on $T$. Then

$$
\left[\left(\omega_{Z}, g_{Z}\right) \bullet_{\mathbb{A}}\left(\omega_{T}, g_{T}\right)\right] \in \mathscr{H}^{p+q}(X \times Y, n+m)_{0}
$$

represents the class of the cycle $z \times t$ in $Z^{p+q}(X \times Y, n+m)_{0}$.

Proof. It follows from [14], Theorem 4.2.3 and [5], Theorem 7.7.

Corollary 5.14. For every $p, q, n, m$, the diagram

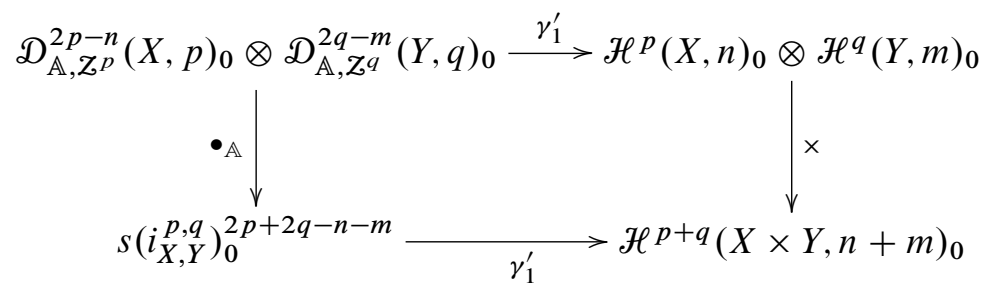

is commutative.

5.3. Product structure on the higher arithmetic Chow groups. Once we have defined a compatible product on each of the complexes involved, the product on the higher arithmetic Chow groups is given by the following diagram. 


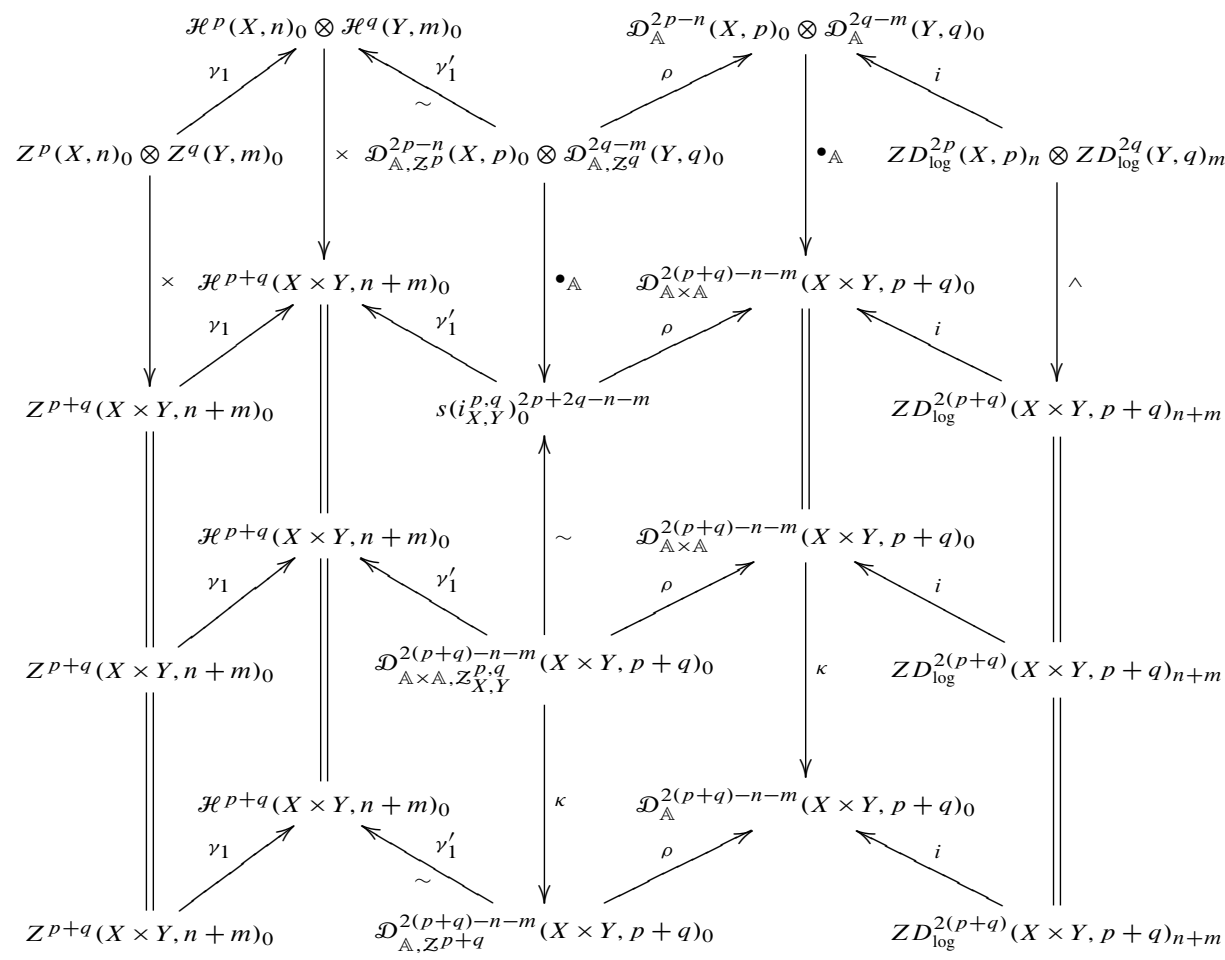

Observe that, in the first set of vertical arrows is where the product is defined, in the second set of vertical arrows we are just inverting the quasi-isomorphism (5.12), finally in the last set of vertical arrows we are applying the morphism $\kappa$.

The above diagram induces a morphism in the derived category of chain complexes

$$
s\left(\widehat{\mathcal{Z}}^{p}(X, *)_{0} \otimes \hat{\mathfrak{Z}}^{q}(Y, *)_{0}\right) \stackrel{\cup}{\rightarrow} s\left(\hat{\mathfrak{Z}}^{p+q}(X \times Y, *)_{0}\right)=\widehat{Z}^{p+q}(X \times Y, *)_{0} .
$$

Recall here the notation we are using, the symbol $\hat{\mathcal{Z}}^{p}(X, *)_{0}$ denotes the diagram where the symbol $\hat{Z}^{p}(X, *)_{0}$ denotes the associated simple complex.

By $\S 1.4$, for any $\beta \in \mathbb{Z}$ there is a morphism $\star \beta$ :

$$
\widehat{Z}^{p}(X, *)_{0} \otimes \widehat{Z}^{q}(Y, *)_{0} \stackrel{\star \beta}{\longrightarrow} s\left(\hat{Z}^{p}(X, *)_{0} \otimes \hat{Z}^{q}(Y, *)_{0}\right) .
$$

The composition of $\star \beta$ with $\cup$ induces a product

$$
\widehat{\mathrm{CH}}^{p}(X, n) \otimes \widehat{\mathrm{CH}}^{q}(Y, m) \stackrel{\cup}{\rightarrow} \widehat{\mathrm{CH}}^{p+q}(X \times Y, n+m),
$$

independent of $\beta$. 
Finally the pull-back by the diagonal map $X \stackrel{\Delta}{\rightarrow} X \times X$ gives an internal product on $\widehat{\mathrm{CH}}^{p}(X, *)$ :

$$
\widehat{\mathrm{CH}}^{p}(X, n) \otimes \widehat{\mathrm{CH}}^{q}(X, m) \stackrel{\cup}{\rightarrow} \widehat{\mathrm{CH}}^{p+q}(X \times X, n+m) \stackrel{\Delta^{*}}{\rightarrow} \widehat{\mathrm{CH}}^{p+q}(X, n+m) .
$$

Thus, in the derived category of complexes, the product is given by the composition

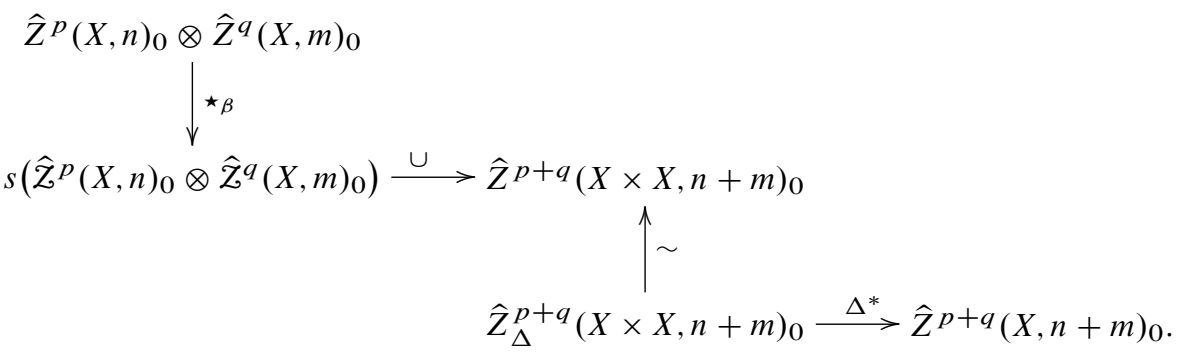

Remark 5.15. It follows from the definition that, for $n=0$, the product $\cup$ agrees with the product on the arithmetic Chow group $\widehat{\mathrm{CH}}^{p}(X)$ defined in [5].

5.4. Commutativity of the product. Let $X, Y$ be arithmetic varieties over a field $K$. We prove here that the pairing defined in the previous subsection on the higher arithmetic Chow groups is commutative, in the sense detailed below.

We first introduce some notation:

- If $B_{*}, C_{*}$ are chain complexes, let

$$
\sigma: s\left(B_{*} \otimes C_{*}\right) \rightarrow s\left(C_{*} \otimes B_{*}\right)
$$

be the map sending $b \otimes c \in B_{n} \otimes C_{m}$ to $(-1)^{n m} c \otimes b \in C_{m} \otimes B_{n}$.

- Let $\sigma_{X, Y}$ be the morphism

$$
\sigma_{X, Y}: Y \times X \rightarrow X \times Y
$$

interchanging $X$ with $Y$.

We will prove that there is a commutative diagram

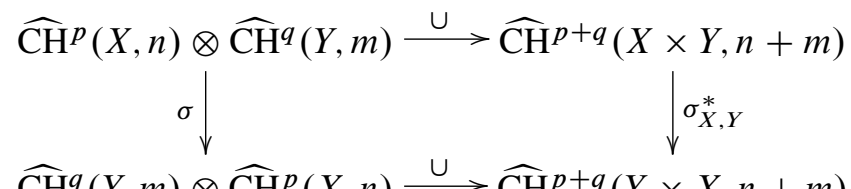

In particular, the internal product on the higher arithmetic Chow groups will be 
graded commutative with respect to the degree $n$. That is, if $W \in \widehat{\mathrm{CH}}^{p}(X, n)$ and $Z \in \widehat{\mathrm{CH}}^{q}(X, m)$, then

$$
W \cup Z=(-1)^{n m} Z \cup W
$$

Recall that, by definition, the product factorizes as

$\widehat{\mathrm{CH}}^{p}(X, n) \otimes \widehat{\mathrm{CH}}^{q}(Y, m) \stackrel{\star_{\beta}}{\longrightarrow} H_{n+m}\left(s\left(\widehat{\mathcal{Z}}^{p}(X, *)_{0} \otimes \widehat{\mathrm{Z}}^{q}(Y, *)_{0}\right)\right) \stackrel{\cup}{\rightarrow} \widehat{\mathrm{CH}}^{p+q}(X \times Y, n+m)$.

By Lemma 1.8, this factorization is independent on the integer $\beta$. Moreover, there is a commutative diagram

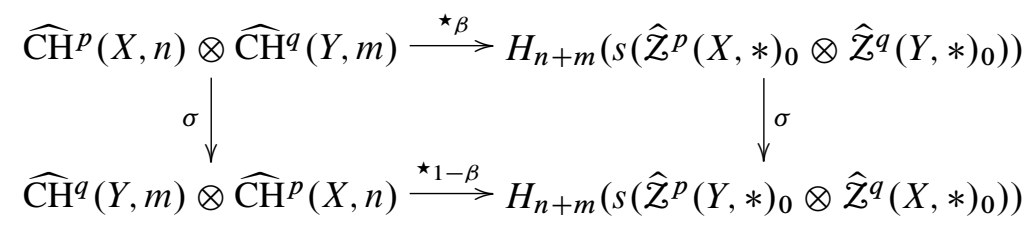

Therefore, all that remains is to check the commutativity for

$$
s\left(\widehat{Z}^{p}(X, *)_{0} \otimes \widehat{Z}^{q}(Y, *)_{0}\right) \stackrel{\cup}{\cup} \widehat{Z}^{p+q}(X \times Y, *)_{0} .
$$

Hence, we want to see that, in the derived category of chain complexes, there is a commutative diagram

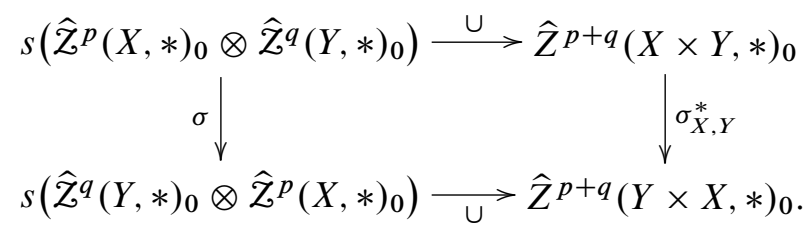

The obstruction to strict commutativity comes from the change of coordinates

$$
\begin{gathered}
\square^{n+m}=\square^{m} \times \square^{n} \stackrel{\sigma_{n, m}}{\longrightarrow} \square^{n} \times \square^{m}=\square^{n+m}, \\
\left(y_{1}, \ldots, y_{m}, x_{1}, \ldots, x_{n}\right) \stackrel{\longmapsto}{\longmapsto}\left(x_{1}, \ldots, x_{n}, y_{1}, \ldots, y_{m}\right) .
\end{gathered}
$$

Recall that the product is described by the big diagram in $\S 5.3$. In order to prove the commutativity, we change the second and third row diagrams of this big diagram, by more suitable diagrams. These changes do not modify the definition of the product, but ease the study of the commutativity.

We define a complex $Z_{\mathbb{A} \times \mathbb{A}}^{p}(X, n)_{0}$ analogously to the definition of the complex $D_{\mathbb{A} \times \mathbb{A}}^{*}(X, p)_{0}($ see $§ 5.2)$. Let

$$
Z^{p}(X, n, m)_{0}:=Z^{p}(X, n+m)_{0},
$$


and let $\delta^{\prime}=\sum_{i=1}^{n}(-1)^{i} \delta_{i}^{0}$ and $\delta^{\prime \prime}=\sum_{i=n+1}^{n+m}(-1)^{i-n} \delta_{i}^{0}$. Then

$$
\left(Z^{p}(X, *, *)_{0}, \delta^{\prime}, \delta^{\prime \prime}\right)
$$

is a 2 -iterated chain complex. For the sake of simplicity, we denote both $\delta^{\prime}$ and $\delta^{\prime \prime}$ by $\delta$.

Denote by $Z_{\mathbb{A} \times \mathbb{A}}^{p}(X, *)_{0}$ the associated simple complex, and analogously define the complex $\mathscr{H}_{\mathbb{A} \times \mathbb{A}}^{p}(X, *)_{0}$.

Let $\widehat{\mathcal{Z}}_{\mathbb{A} \times \mathbb{A}}^{p, q}(X \times Y, *)_{0}$ be the diagram

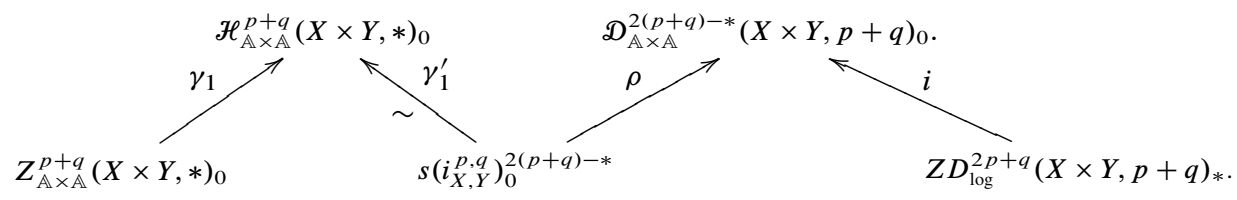

This diagram will fit in the second row of the new big diagram. Denote by $\widehat{Z}_{\mathbb{A} \times \mathbb{A}}^{p, q}(X \times Y, *)_{0}$ the simple complex associated to this diagram.

The third row of the new big diagram corresponds to a diagram whose complexes are obtained from the refined normalized complex of Definition 1.18. The fact that, in these complexes, most of the face maps vanish is the key point to construct explicit homotopies for the commutativity of the product. So, consider the following complexes:

- Let $Z^{q}(X, *, *)_{00}$ be the 2 -iterated chain complex with

$$
Z^{q}(X, n, m)_{00}:=\bigcap_{i \neq 0, n+1} \operatorname{ker} \delta_{i}^{0} \subset Z^{q}(X, n+m)_{0},
$$

and with differentials $\left(\delta^{\prime}, \delta^{\prime \prime}\right)=\left(-\delta_{1}^{0},-\delta_{n+1}^{0}\right)$. Denote by $Z_{\mathbb{A} \times \mathbb{A}}^{q}(X, *)_{00}$ the associated simple complex.

- Let $\tau \mathscr{D}_{\log }^{*}\left(X \times \square^{*} \times \square^{*}, p\right)_{00}$ be the 3 -iterated complex whose $(r,-n,-m)$ graded piece is

$$
\tau \mathscr{D}_{\log }^{r}\left(X \times \square^{n} \times \square^{m}, p\right)_{00}=\bigcap_{i \neq 0, n+1} \operatorname{ker} \delta_{i}^{0} \subset \tau \mathscr{D}_{\log }^{r}\left(X \times \square^{n+m}, p\right)_{0},
$$

and with differentials $\left(d_{\mathscr{D}},-\delta_{1}^{0},-\delta_{n+1}^{0}\right)$. Let $\mathscr{D}_{\mathbb{A} \times \mathbb{A}}^{*}(X, p)_{00}$ be the associated simple complex.

- Let $\tau D_{\log , Z_{X, Y, *, *}^{p, q}}^{*}\left(X \times Y \times \square^{*} \times \square^{*}, p+q\right)_{00}$ be the 3-iterated complex with

$$
\tau D_{\log , Z_{X, Y, n, m}^{p, q}}^{r}\left(X \times Y \times \square^{n} \times \square^{m}, p+q\right)_{00}=\bigcap_{i \neq 0, n+1} \operatorname{ker} \delta_{i}^{0}
$$


as a subset of $\tau \mathcal{D}_{\log , Z_{X, Y, n, m}^{r, q}}^{r}\left(X \times Y \times \square^{n+m}, p+q\right)_{0}$. The differentials are given by $\left(d_{\mathscr{D}},-\delta_{1}^{0},-\delta_{n+1}^{0}\right)$. Let $\mathcal{D}_{\mathbb{A} \times \mathbb{A}, \mathcal{Z}_{X, Y}^{p, q}}^{*}(X \times Y, p)_{00}$ be the associated simple complex.

Remark 5.18. Observe that there are induced morphisms

$$
\begin{gathered}
Z_{\mathbb{A} \times \mathbb{A}}^{p+q}(X \times Y, *)_{00} \stackrel{\gamma_{1}}{\longrightarrow} \mathcal{H}_{\mathbb{A} \times \mathbb{A}}^{p+q}(X \times Y, *)_{00}, \\
\mathcal{D}_{\mathbb{A} \times \mathbb{A}, Z_{X, Y}^{p, q}}^{2(p+q)-*}(X \times Y, p+q)_{00} \stackrel{\gamma_{1}^{\prime}}{\longrightarrow} \mathcal{H}_{\mathbb{A} \times \mathbb{A}}^{p+q}(X \times Y, *)_{00}, \\
\mathcal{D}_{\mathbb{A} \times \mathbb{A}, Z_{X, Y}^{p, q}}^{2(p+q)-*}(X \times Y, p+q)_{00} \stackrel{\rho}{\longrightarrow} \mathcal{D}_{\mathbb{A} \times \mathbb{A}}^{2(p+q)-*}(X \times Y, p+q)_{00} .
\end{gathered}
$$

Let $\hat{Z}_{\mathbb{A} \times \mathbb{A}}^{p, q}(X \times Y, *)_{00}$ be the diagram

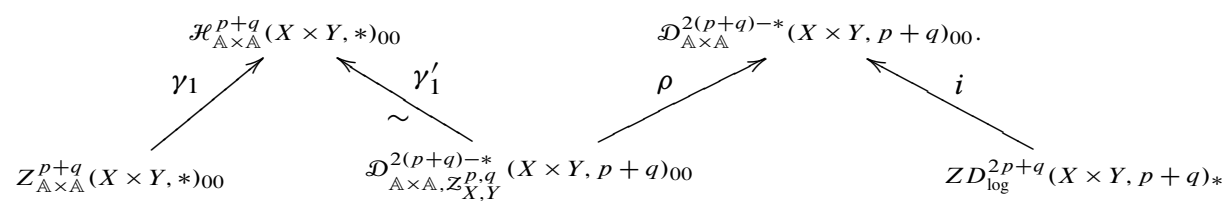

This is the diagram fitting in the third row of the new diagram. Let $\widehat{Z}_{\mathbb{A} \times \mathbb{A}}^{p, q}(X \times Y, *)_{00}$ be the simple complex associated to this diagram.

Lemma 5.19. Let $X$ be an arithmetic variety over a field.

(i) The natural chain morphisms

$$
\begin{gathered}
Z_{\mathbb{A} \times \mathbb{A}}^{q}(X, *)_{00} \stackrel{i}{\rightarrow} Z_{\mathbb{A} \times \mathbb{A}}^{q}(X, *)_{0}, \\
Z_{\mathbb{A} \times \mathbb{A}}^{q}(X, *)_{0} \stackrel{\kappa}{\longrightarrow} Z^{q}(X, *)_{0},
\end{gathered}
$$

are quasi-isomorphisms.

(ii) The natural cochain morphisms

$$
\begin{gathered}
\mathcal{D}_{\mathbb{A} \times \mathbb{A}}^{*}(X, p)_{00} \stackrel{i}{\rightarrow} D_{\mathbb{A} \times \mathbb{A}}^{*}(X, p)_{0}, \\
\mathcal{D}_{\mathbb{A} \times \mathbb{A}, Z_{X, Y}^{p, q}}^{*}(X \times Y, p+q)_{00} \stackrel{i}{\rightarrow} \mathcal{D}_{\mathbb{A} \times \mathbb{A}, Z_{X, Y}^{p, q}}^{*}(X \times Y, p+q)_{0}, \\
\mathcal{D}_{\mathbb{A} \times \mathbb{A}}^{*}(X, p)_{0} \stackrel{\kappa}{\longrightarrow} \mathcal{D}_{\mathbb{A}}^{*}(X, p)_{0},
\end{gathered}
$$

are quasi-isomorphisms.

Proof. The proofs of the facts that the morphisms $i$ are quasi-isomorphisms are analogous for the three cases. For every $n, m$, let $B(n, m)$ denote either $Z^{p}(X, n, m)$, 
$\tau \mathscr{D}_{\log }^{r}\left(X \times \square^{n} \times \square^{m}, p\right)$ or $\tau \mathscr{D}_{\log , \mathcal{L}_{X, Y, n, m}^{p, q}}^{r}\left(X \times Y \times \square^{n} \times \square^{m}, p+q\right)$, for some $r$. The groups $B(n, m)_{0}$ and $B(n, m)_{00}$ are defined analogously.

Observe that for every $n, m, B(\cdot, m)$ and $B(n, \cdot)$ are cubical abelian groups. We want to see that there is a quasi-isomorphism

$$
s\left(N_{0}^{2} N_{0}^{1} B(*, *)\right) \stackrel{i}{\rightarrow} s\left(N^{2} N^{1} B(*, *)\right),
$$

where the superscript 1 refers to the cubical structure given by the first index $n$ and the superscript 2 to the cubical structure given by the second index $m$. A spectral sequence argument together with Lemma 1.20 and Proposition 1.24 show that there is a quasi-isomorphism $s\left(N^{2} N_{0}^{1} B(*, *)\right) \stackrel{\sim}{\rightarrow} s\left(N^{2} N^{1} B(*, *)\right)$. By Lemma 1.20 and an spectral sequence argument again, we obtain that there is a quasi-isomorphism $s\left(N_{0}^{2} N_{0}^{1} B(*, *)\right) \stackrel{i}{\rightarrow} s\left(N^{2} N_{0}^{1} B(*, *)\right)$. Therefore, (5.25) is a quasi-isomorphism.

The proofs of the facts that the morphisms in (5.21) and (5.24) are quasi-isomorphisms are analogous to each other. Therefore, we just prove the statement for the morphism (5.21). Consider the composition morphism

$$
j: Z^{q}(X, m)_{0} \rightarrow Z^{q}(X, 0, m)_{0} \rightarrow Z_{\mathbb{A} \times \mathbb{A}}^{q}(X, m)_{0} .
$$

The composition of morphisms $Z^{q}(X, m)_{0} \stackrel{j}{\rightarrow} Z_{\mathbb{A} \times \mathbb{A}}^{q}(X, m)_{0} \stackrel{\kappa}{\rightarrow} Z^{q}(X, m)_{0}$ is the identity. Hence, it is enough to see that $j$ is a quasi-isomorphism. Consider the 1st quadrant spectral sequence with

$$
E_{n, m}^{1}=H_{m}\left(Z^{q}(X, n, *)_{0}\right) .
$$

We will see that if $n \geq 1, E_{n, m}^{1}=0$. By the homotopy invariance of higher Chow groups, the map

$$
f: Z^{q}\left(X \times \square^{n}, *\right)_{0} \stackrel{\delta_{1}^{1} \cdots \delta_{1}^{1}}{\longrightarrow} Z^{q}(X, *)_{0}
$$

is a quasi-isomorphism. By Proposition 1.24, it induces a quasi-isomorphism

$$
f: Z^{q}\left(X \times \square^{n}, *\right)_{0}=N Z^{q}\left(X \times \square^{n}, *\right)_{0} \rightarrow N Z^{q}(X, *)_{0}
$$

where the cubical structure on $Z^{q}(X, *)_{0}$ is the trivial one. Since for a trivial cubical abelian group $N Z^{q}(X, *)_{0}=0$, we see that

$$
H_{m}\left(Z^{q}(X, n, *)_{0}\right)=0, \quad n>0,
$$

and hence

$$
E_{n, m}^{1}= \begin{cases}0 & \text { if } n>0 \\ \mathrm{CH}^{q}(X, m) & \text { if } n=0\end{cases}
$$


It follows from the lemma that the product on the higher arithmetic Chow groups is also represented by the following diagram of complexes:

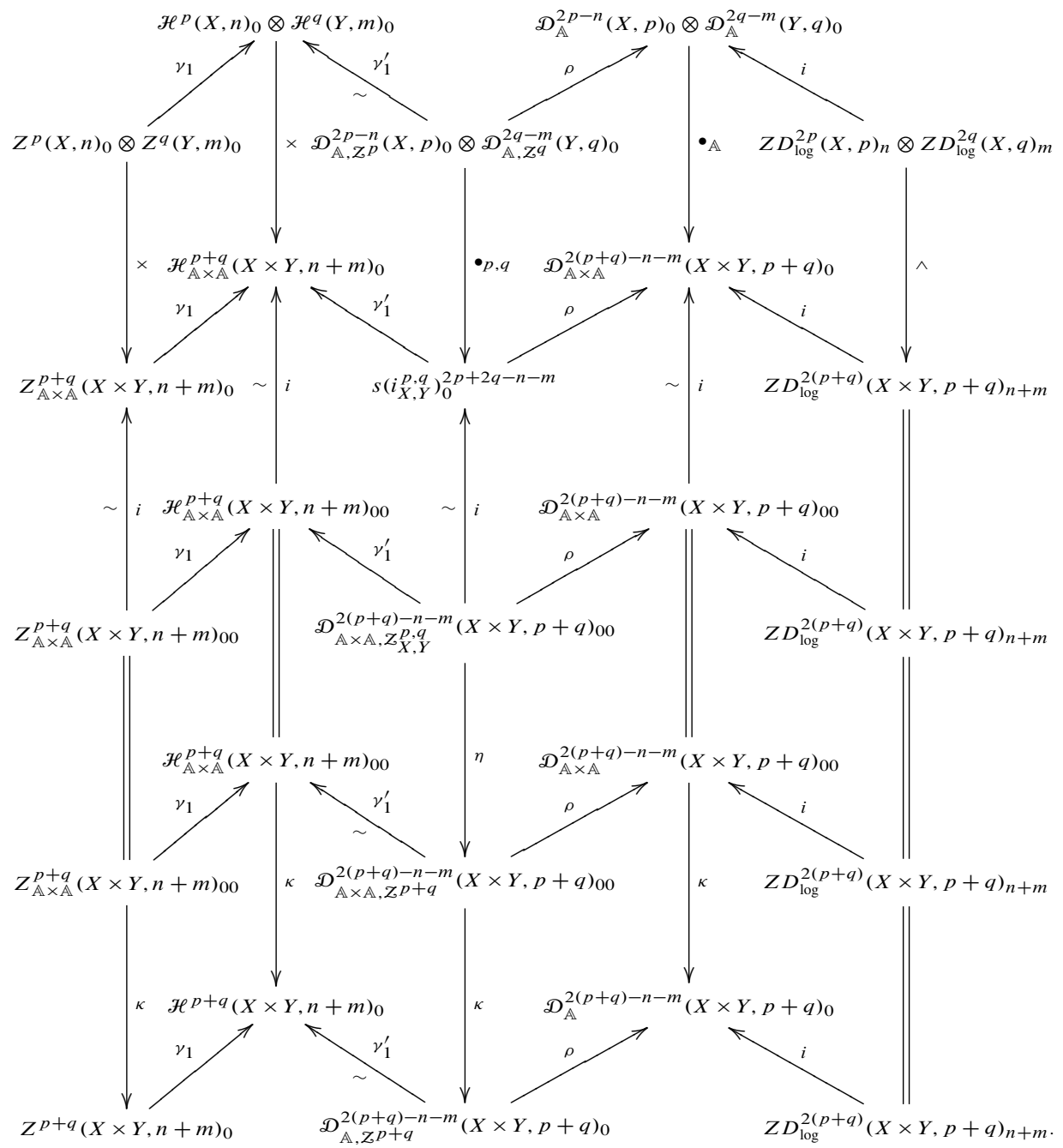

In the first set of vertical arrows of this diagram is where the product is defined. In the second set of vertical rows we invert the quasi-isomorphisms that relate the normalized complex and the refined normalized complex. Moreover, we also invert the quasi-isomorphism analogous to (5.12). In the third set of vertical arrows we just consider the change of supports $\mathcal{Z}_{X, Y}^{p, q} \subset \mathfrak{Z}^{p+q}$. We will denote the map induced by this change of support by $\eta$. Finally in the last set of vertical arrows we apply the morphisms $\kappa$ induced by the identification $\square^{n} \times \square^{m}=\square^{n+m}$. 
Let $\widehat{Z}_{\mathbb{A} \times \mathbb{A}}^{p+q}(X \times Y, *)_{00}$ denote the simple of the diagram of the fourth row. Hence, in the derived category of complexes, this product is described by the composition

$$
\begin{aligned}
& s\left(\widehat{\mathcal{Z}}^{p}(X, *)_{0} \otimes \widehat{\mathcal{Z}}^{q}(Y, *)_{0}\right) \stackrel{\cup}{\longrightarrow} \widehat{Z}_{\mathbb{A} \times \mathbb{A}}^{p, q}(X \times Y, *)_{0} \\
& \wedge \\
& \text { I } i \\
& \hat{Z}_{\mathbb{A} \times \mathbb{A}}^{p, q}(X \times Y, *)_{00} \stackrel{\eta}{\longrightarrow} \widehat{Z}_{\mathbb{A} \times \mathbb{A}}^{p+q}(X \times Y, *)_{00} \\
& \downarrow \\
& \widehat{Z}^{p+q}(X \times Y, *)_{0} \text {. }
\end{aligned}
$$

Note that the difference between the complexes $\widehat{Z}_{\mathbb{A} \times \mathbb{A}}^{p, q}(X \times Y, *)_{00}$ and $\widehat{Z}_{\mathbb{A} \times \mathbb{A}}^{p+q}(X \times$ $Y, *)_{00}$ lies on the change of supports $Z_{X, Y}^{p, q} \subset Z^{p+q}$. This is indicated by either two codimension superscripts $p, q$ in the first one or a unique codimension superscript $p+q$ in the second.

We next use this description of the product in the higher arithmetic Chow groups in order to prove its commutativity.

Recall that the map $\sigma_{n, m}$ is defined by

$$
\begin{gathered}
\square^{n+m}=\square^{m} \times \square^{n} \stackrel{\sigma_{n, m}}{\longrightarrow} \square^{n} \times \square^{m}=\square^{n+m}, \\
\left(y_{1}, \ldots, y_{m}, x_{1}, \ldots, x_{n}\right) \stackrel{\longmapsto}{\longmapsto}\left(x_{1}, \ldots, x_{n}, y_{1}, \ldots, y_{m}\right) .
\end{gathered}
$$

Let

$$
\sigma_{X, Y, n, m}: Y \times X \times \square^{m} \times \square^{n} \rightarrow X \times Y \times \square^{n} \times \square^{m}
$$

be the map $\sigma_{X, Y} \times \sigma_{n, m}$.

We define a morphism of diagrams

$$
\hat{\mathcal{Z}}_{\mathbb{A} \times \mathbb{A}}^{p, q}(X \times Y, *)_{0} \stackrel{\sigma_{X, Y, \square}^{*}}{\longrightarrow} \hat{\mathcal{Z}}_{\mathbb{A} \times \mathbb{A}}^{q, p}(Y \times X, *)_{0}
$$

as follows:

- Let $\sigma_{X, Y, \square}^{*}: Z_{\mathbb{A} \times \mathbb{A}}^{p+q}(X \times Y, *)_{0} \rightarrow Z_{\mathbb{A} \times \mathbb{A}}^{p+q}(Y \times X, *)_{0}$ be the map sending

$$
Z \in Z^{p+q}(X \times Y, n, m)_{0} \text { to }(-1)^{n m} \sigma_{X, Y, n, m}^{*}(Z) \in Z^{p+q}(Y \times X, m, n)_{0} .
$$

The morphism $\sigma_{X, Y, \square}^{*}: \mathscr{H}_{\mathbb{A} \times \mathbb{A}}^{p+q}(X \times Y, *)_{0} \rightarrow \mathscr{H}_{\mathbb{A} \times \mathbb{A}}^{p+q}(Y \times X, *)_{0}$ is defined analogously.

- Let $\sigma_{X, Y, \square}^{*}: \mathcal{D}_{\mathbb{A} \times \mathbb{A}}^{*}(X \times Y, p+q)_{0} \rightarrow \mathscr{D}_{\mathbb{A} \times \mathbb{A}}^{*}(Y \times X, p+q)_{0}$ be the map that, at the $(*,-n,-m)$ component, is

$(-1)^{n m} \sigma_{X, Y, n, m}^{*}: \tau \mathscr{D}_{\log }^{*}\left(X \times Y \times \square^{n} \times \square^{m}, p+q\right)_{0} \rightarrow \tau \mathscr{D}_{\log }^{*}\left(Y \times X \times \square^{m} \times \square^{n}, p+q\right)_{0}$.

Observe that it is a cochain morphism. 
- We define analogously the morphism $\sigma_{X, Y, \square}^{*}: s\left(i_{X, Y}^{p, q}\right)_{0}^{*} \rightarrow s\left(i_{Y, X}^{q, p}\right)_{0}^{*}$.

These morphisms commute with the morphisms $\gamma_{1}, \gamma_{1}^{\prime}$ and $\rho$. Hence, they induce a morphism of diagrams and therefore a morphism on the associated simple complexes:

$$
\widehat{Z}_{\mathbb{A} \times \mathbb{A}}^{p, q}(X \times Y, *)_{0} \stackrel{\sigma_{X, Y, \square}^{*}}{\longrightarrow} \widehat{Z}_{\mathbb{A} \times \mathbb{A}}^{q, p}(Y \times X, *)_{0} .
$$
$Y, *)_{00}$.

Note that the morphism $\sigma_{X, Y, \square}^{*}$ restricts to $\hat{Z}_{\mathbb{A} \times \mathbb{A}}^{p, q}(X \times Y, *)_{00}$ and to $\hat{Z}_{\mathbb{A} \times \mathbb{A}}^{p+q}(X \times$

Lemma 5.26. The diagram

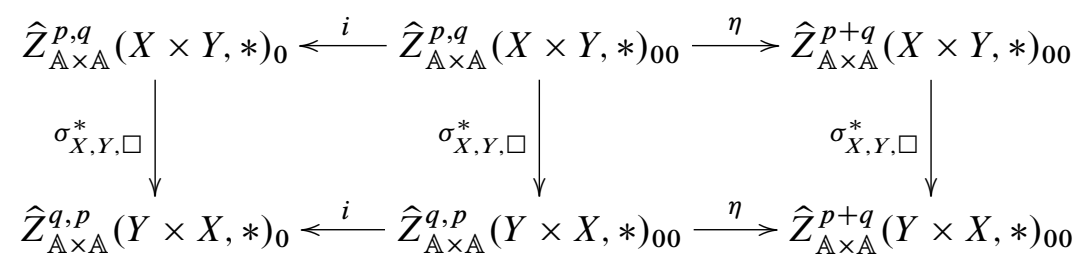

is commutative.

Proof. The statement follows from the definitions.

Lemma 5.27. The diagram

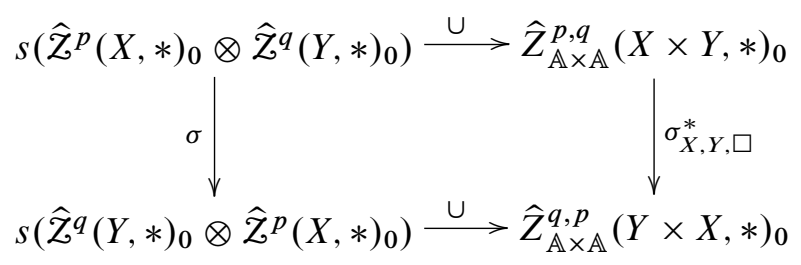

is commutative.

Proof. It follows from the definition that the morphism $\sigma_{X, Y, \square}^{*}$ commutes with the product $\times$ in $Z^{*}(X, *)_{0}$ and in $\mathscr{H}^{*}(X, *)_{0}$. The fact that it commutes with $\bullet_{\mathbb{A}}$ and $\bullet p, q$ is an easy computation.

By Lemmas 5.26 and 5.27, we are left to see that the diagram

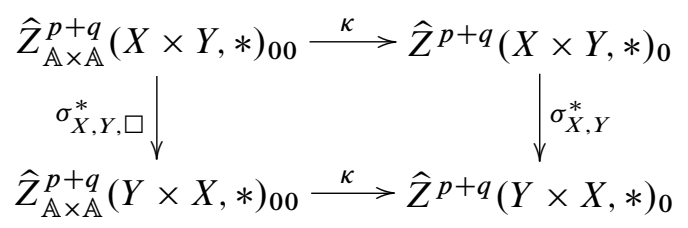


is commutative up to homotopy. We follow the ideas used by Levine, in [19], §4, in order to prove the commutativity of the product on the higher algebraic Chow groups. We will end up with an explicit homotopy for the commutativity of diagram 5.28.

Remark 5.29. For any scheme $X$, consider the morphism

$$
\hat{Z}_{\mathbb{A} \times \mathbb{A}}^{p}(X, *)_{00} \stackrel{\sigma_{\square}^{*}}{\longrightarrow} \hat{Z}_{\mathbb{A} \times \mathbb{A}}^{p}(X, *)_{00}
$$

induced by $(-1)^{n m} \sigma_{n, m}^{*}$ at each component. Then $\sigma_{X, Y, \square}^{*}=\sigma_{X, Y}^{*} \sigma_{\square}^{*}$ and hence, the commutativity of the diagram (5.28) will follow from the commutativity (up to homotopy) of the diagram

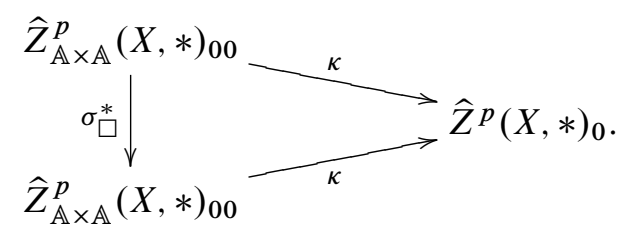

Let $W_{n}$ be the closed subvariety of $\square^{n+1} \times \mathbb{P}^{1}$ defined by the equation

$$
t_{1}\left(1-x_{1}\right)\left(1-x_{n+1}\right)=t_{1}-t_{0},
$$

where $\left(t_{0}: t_{1}\right)$ are the coordinates in $\mathbb{P}^{1}$ and $\left(x_{1}, \ldots, x_{n+1}\right)$ are the coordinates in $\square^{n+1}$. Recall that we have identified $\square^{1}$ with the subset $t_{0} \neq t_{1}$ of $\mathbb{P}^{1}$, with coordinate $x=t_{0} / t_{1}$. Then there is an isomorphism $W_{n} \cong \square^{n} \times \square^{1}$. The inverse of this isomorphism is given by

$$
\begin{aligned}
\square^{n+1} & \stackrel{\varphi_{n}}{\longrightarrow} W_{n}, \\
\left(x_{1}, \ldots, x_{n+1}\right) & \longmapsto\left(x_{1}, \ldots, x_{n+1}, x_{1}+x_{n+1}-x_{1} x_{n+1}\right) .
\end{aligned}
$$

Consider the projection

$$
\pi_{n}: W_{n} \rightarrow \square^{n}, \quad\left(x_{1}, \ldots, x_{n+1}, t\right) \mapsto\left(x_{2}, \ldots, x_{n}, t\right) .
$$

Let $\tau$ be the permutation

$$
\square^{n} \stackrel{\tau}{\rightarrow} \square^{n}, \quad\left(x_{1}, \ldots, x_{n}\right) \mapsto\left(x_{2}, \ldots, x_{n}, x_{1}\right) .
$$

Remark 5.31. Let $\sigma_{n, m}$ be the map defined in (5.17). Observe that it is decomposed as $\sigma_{n, m}=\tau \circ \stackrel{m}{.} \circ \circ$. Therefore, $\sigma_{n, m}^{*}=\tau^{*} \circ \stackrel{m}{.} \circ \tau^{*}$. 
It is easy to check that the following identities are satisfied:

$$
\begin{aligned}
& \pi_{n} \varphi_{n} \delta_{0}^{i}= \begin{cases}\mathrm{id} & \text { if } i=1, \\
\delta_{0}^{i-1} \pi_{n-1} \varphi_{n-1} & \text { if } i=2, \ldots, n, \\
\tau & \text { if } i=n+1 ;\end{cases} \\
& \pi_{n} \varphi_{n} \delta_{1}^{i}= \begin{cases}\delta_{1}^{n} \sigma^{n} & \text { if } i=1, \\
\delta_{1}^{i-1} \pi_{n-1} \varphi_{n-1} & \text { if } i=2, \ldots, n, \\
\delta_{1}^{n} \sigma^{n} \tau & \text { if } i=n+1 .\end{cases}
\end{aligned}
$$

Let $W_{n}^{X}$ be the pull-back of $W_{n}$ to $X \times \square^{n}$. Then the maps

$$
\pi_{n}: W_{n}^{X} \rightarrow X \times \square^{n}, \quad \text { and } \quad \varphi_{n}: X \times \square^{n+1} \rightarrow W_{n}^{X}
$$

are defined accordingly.

Proposition 5.33. Let $X$ be a quasi-projective regular scheme over a field $k$.

(i) The scheme $W_{n}$ is a flat regular scheme over $\square^{n}$.

(ii) There is a well-defined map

$$
Z^{p}(X, n) \stackrel{h_{n}}{\longrightarrow} Z^{p}(X, n+1), \quad Y \mapsto \varphi_{n}^{*} \pi_{n}^{*}(Y)
$$

Proof. See [19], Lemma 4.1.

For every $n \geq 1$, we define the morphisms

$$
\begin{gathered}
\mathscr{H}^{p}(X, n) \stackrel{h_{n}}{\longrightarrow} \mathscr{H}^{p}(X, n+1), \\
\tau \mathcal{D}_{\log }^{*}\left(X \times \square^{n}, p\right) \stackrel{h_{n}}{\longrightarrow} \tau \mathcal{D}_{\log }^{*}\left(X \times \square^{n+1}, p\right), \\
\tau \mathscr{D}_{\log , Z^{p}}^{*}\left(X \times \square^{n}, p\right) \stackrel{h_{n}}{\longrightarrow} \tau \mathcal{D}_{\log , Z^{p}}^{*}\left(X \times \square^{n+1}, p\right),
\end{gathered}
$$

by $h_{n}=\varphi_{n}^{*} \pi_{n}^{*}$. By Proposition 5.33, (ii), these morphisms are well defined.

Lemma 5.34. Let $\alpha$ be an element of $Z^{q}(X, n)_{0}, \mathscr{H}^{p}(X, n)_{0}, \tau \mathscr{D}_{\log , Z^{p}}^{*}\left(X \times \square^{n}, p\right)_{0}$ or $\tau \mathcal{D}_{\log }^{*}\left(X \times \square^{n}, p\right)_{0}$. Then the following equality is satisfied:

$$
\delta h_{n}(\alpha)+\sum_{i=1}^{n-1}(-1)^{i} h_{n-1} \delta_{i}^{0}(\alpha)=-\alpha+(-1)^{n+1} \tau^{*}(\alpha)
$$


Proof. By hypothesis, $\delta_{i}^{1}(\alpha)=0$ for all $i=1, \ldots, n$. Then, by the pull-back of the equalities (5.32), we see that $\delta_{i}^{1} \varphi_{n}^{*} \pi_{n}^{*}(\alpha)=0$. Therefore, using (5.32),

$$
\begin{aligned}
\delta h_{n}(\alpha) & =\sum_{i=1}^{n+1} \sum_{j=0,1}(-1)^{i+j} \delta_{i}^{j} \varphi_{n}^{*} \pi_{n}^{*}(\alpha)=\sum_{i=1}^{n+1}(-1)^{i} \delta_{i}^{0} \varphi_{n}^{*} \pi_{n}^{*}(\alpha) \\
& =-\alpha+\sum_{i=2}^{n}(-1)^{i} \varphi_{n}^{*} \pi_{n-1}^{*} \delta_{i-1}^{0}(\alpha)+(-1)^{n-1} \tau^{*}(\alpha) \\
& =-\alpha-\sum_{i=1}^{n-1}(-1)^{i} h_{n-1} \delta_{i}^{0}(\alpha)+(-1)^{n+1} \tau^{*}(\alpha),
\end{aligned}
$$

as desired.

Proposition 5.35. Let $X$ be an arithmetic variety over a field. Then the following diagram is commutative up to homotopy.

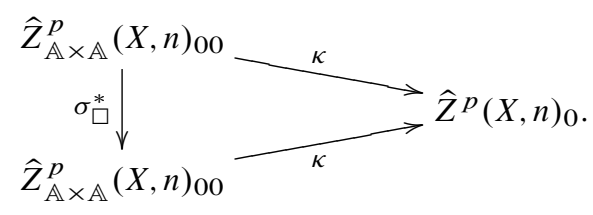

Proof. We start by defining maps

$$
\begin{gathered}
Z^{p}(X, n, m)_{00} \stackrel{H_{n, m}}{\longrightarrow} Z^{p}(X, n+m+1)_{0}, \\
\mathscr{H}^{p}(X, n, m)_{00} \stackrel{H_{n, m}}{\longrightarrow} \mathcal{H}^{p}(X, n+m+1)_{0}, \\
\tau \mathcal{D}_{\log }^{*}\left(X \times \square^{n} \times \square^{m}, p\right)_{00} \stackrel{H_{n, m}}{\longrightarrow} \tau \mathcal{D}_{\log }^{*}\left(X \times \square^{n+m+1}, p\right)_{0}, \\
\tau \mathscr{D}_{\log , Z^{p}}^{*}\left(X \times \square^{n} \times \square^{m}, p\right)_{00} \stackrel{H_{n, m}}{\longrightarrow} \tau \mathcal{D}_{\log , Z^{p}}^{*}\left(X \times \square^{n+m+1}, p\right)_{0} .
\end{gathered}
$$

By construction, these maps will commute with $\gamma_{1}, \gamma_{1}^{\prime}$ and $\rho$. This will allow us to define the homotopy for the commutativity of the diagram in the statement.

All the maps $H_{n, m}$ will be defined in the same way. Thus, let $B(X, n, m)_{00}$ denote either $Z^{p}(X, n, m)_{00}, \mathscr{H}^{p}(X, n, m)_{00}, \tau \mathscr{D}_{\log }^{*}\left(X \times \square^{n} \times \square^{m}, p\right)_{00}$, or $\tau \mathscr{D}_{\log , Z^{p}}^{*}(X \times$ $\left.\square^{n} \times \square^{m}, p\right)_{00}$. For the last two cases, $B(X, n, m)_{00}$ is a cochain complex, while for the first two cases, it is a group. Analogously, denote by $B(X, n+m+1)_{0}$ the groups/complexes that are the target of $H_{n, m}$. The map $H_{n, m}$ will be a cochain complex for the last two cases.

Let $\alpha \in B(X, n, m)_{00}$. Then let $H_{n, m}(\alpha) \in B(X, n+m+1)_{0}$ be defined by

$$
H_{n, m}(\alpha)= \begin{cases}\sum_{i=0}^{n-1}(-1)^{(m+i)(n+m-1)} h_{n+m+1}\left(\left(\tau^{*}\right)^{m+i}(\alpha)\right), & n \neq 0, \\ 0, & n=0 .\end{cases}
$$


From the definition it follows that:

$\triangleright$ If $B(X, n, m)_{00}$ is $\tau \mathscr{D}_{\log }^{*}\left(X \times \square^{n} \times \square^{m}, p\right)_{00}$, or $\tau \mathscr{D}_{\log , Z^{p}}^{*}\left(X \times \square^{n} \times \square^{m}, p\right)_{00}$, then

$$
d_{\mathscr{D}} H_{n, m}(\alpha)=H_{n, m} d_{\mathscr{D}}(\alpha),
$$

i.e. $H_{n, m}$ is a cochain morphism.

$\triangleright \gamma_{1} H_{n, m}=H_{n, m} \gamma_{1}, \gamma_{1}^{\prime} H_{n, m}=H_{n, m} \gamma_{1}^{\prime}$ and $\rho H_{n, m}=H_{n, m} \rho$.

Recall that in all these complexes,

$$
\begin{aligned}
\delta^{\prime}(\alpha) & =-\delta_{1}^{0}(\alpha) \in B(X, n-1, m)_{00} \\
\delta^{\prime \prime}(\alpha) & =-\delta_{n+1}^{0}(\alpha) \in B(X, n, m-1)_{00}
\end{aligned}
$$

Lemma 5.37. For every $\alpha \in B(X, n, m)_{00}$ we have

$$
\delta H_{n, m}(\alpha)-H_{n-1, m} \delta_{1}^{0}(\alpha)-(-1)^{n} H_{n, m-1} \delta_{n+1}^{0}(\alpha)=\alpha-(-1)^{n m} \sigma_{n, m}^{*}(\alpha) .
$$

Proof. If $n=0$, since $\alpha=\sigma_{0, m}(\alpha)$ and $H_{0, m}(\alpha)=0$ the equality is satisfied. For simplicity, for every $i=0, \ldots, n-1$, we denote

$$
H_{n, m}^{i}(\alpha)=(-1)^{(m+i)(n+m-1)} h_{n+m+1}\left(\left(\tau^{*}\right)^{m+i}(\alpha)\right) \in B(X, n+m+1)_{0} .
$$

An easy computation shows that

$$
\delta_{j}^{0} \tau^{*}(\alpha)= \begin{cases}\tau^{*} \delta_{j-1}^{0}(\alpha) & \text { if } j \neq 1 \\ \delta_{n}^{0}(\alpha) & \text { if } j=1\end{cases}
$$

and hence

$$
\delta_{j}^{0}\left(\tau^{*}\right)^{i}(\alpha)= \begin{cases}\left(\tau^{*}\right)^{i} \delta_{j-i}^{0}(\alpha) & \text { if } j>i \\ \left(\tau^{*}\right)^{i-1} \delta_{n}^{0}(\alpha) & \text { if } j=i \\ \left(\tau^{*}\right)^{i-1} \delta_{n-i+j}^{0}(\alpha) & \text { if } j<i\end{cases}
$$

Therefore,

$$
\begin{aligned}
\delta H_{n, m}^{i}(\alpha)= & \sum_{j=1}^{n+m+1}(-1)^{j+(m+i)(n+m-1)} \delta_{j}^{0} h_{n+m+1}\left(\left(\tau^{*}\right)^{m+i}(\alpha)\right) \\
= & (-1)^{1+(m+i)(n+m-1)}\left(\tau^{*}\right)^{m+i}(\alpha)+(-1)^{(m+i+1)(n+m-1)}\left(\tau^{*}\right)^{m+i+1}(\alpha) \\
& \quad+\sum_{j=2}^{n+m}(-1)^{j+(m+i)(n+m-1)} h_{n+m}\left(\delta_{j-1}^{0}\left(\tau^{*}\right)^{m+i}(\alpha)\right) .
\end{aligned}
$$

Recall that the only non-zero faces of $\alpha$ are $\delta_{1}^{0}$ and $\delta_{n+1}^{0}$. Therefore, from the equalities (5.32), we see that the only non-zero faces are the faces corresponding to 
the indices $j=m+i+2$ and $j=i+2$. In these cases, they take the values $\left(\tau^{*}\right)^{m+i} \delta_{1}^{0}$ and $\left(\tau^{*}\right)^{m+i-1} \delta_{n+1}^{0}$ respectively. Therefore, if $i \neq n-1$, we obtain

$$
\begin{aligned}
\delta H_{n, m}^{i}(\alpha)=- & (-1)^{(m+i)(n+m-1)}\left(\tau^{*}\right)^{m+i}(Z) \\
& +(-1)^{(m+i+1)(n+m-1)}\left(\tau^{*}\right)^{m+i+1}(\alpha) \\
& +(-1)^{(m+i)(n+m-2)} h_{n+m}\left(\left(\tau^{*}\right)^{m+i} \delta_{1}^{0}(\alpha)\right) \\
& +(-1)^{i+(m+i)(n+m-1)} h_{n+m}\left(\left(\tau^{*}\right)^{m-1+i} \delta_{n+1}^{0}(\alpha)\right) .
\end{aligned}
$$

Observe that $(-1)^{i+(m+i)(n+m-1)}=(-1)^{(m+i-1)(n+m)+n}$. Therefore, the last summand in the previous equality is exactly

$$
H_{n-1, m}^{i}\left(\delta_{1}^{0}(\alpha)\right)+(-1)^{n} H_{n, m-1}^{i}\left(\delta_{n+1}^{0}(\alpha)\right) .
$$

If $i=n-1$, then $\delta_{j-1}^{0}\left(\tau^{*}\right)^{m+i}(\alpha)=0$, for $j=2, \ldots, n-m$. Therefore,

$$
\begin{aligned}
\delta H_{n, m}^{n-1}(\alpha)=( & -1)^{1+(m+n-1)(n+m-1)}\left(\tau^{*}\right)^{m+n-1}(\alpha) \\
& +(-1)^{(m+n)(n+m-1)}\left(\tau^{*}\right)^{m+n}(\alpha) \\
& +(-1)^{n-1+(m+n-1)(n+m-1)} h_{n+m}\left(\left(\tau^{*}\right)^{m-1+i} \delta_{n+1}^{0}(\alpha)\right) \\
=- & (-1)^{(m+n-1)(n+m-1)}\left(\tau^{*}\right)^{m+n-1}(\alpha)+\alpha \\
& +(-1)^{n+(m+n-2)(n+m)} h_{n+m}\left(\left(\tau^{*}\right)^{m-1+i} \delta_{n+1}^{0}(\alpha)\right) .
\end{aligned}
$$

Finally, we have seen that

$$
\begin{aligned}
\delta H_{n, m}(\alpha)=- & (-1)^{m(n+m-1)}\left(\tau^{*}\right)^{m}(\alpha)+\sum_{i=0}^{n-2} H_{n-1, m}^{i}\left(\delta_{1}^{0}(\alpha)\right) \\
& +\sum_{i=0}^{n-1}(-1)^{n} H_{n, m-1}^{i}\left(\delta_{n+1}^{0}(\alpha)\right)+\alpha,
\end{aligned}
$$

and since $(-1)^{m(n+m-1)}=(-1)^{n m}$, we obtain the equality

$$
\delta H_{n, m}(\alpha)-H_{n-1, m}\left(\delta_{1}^{0}(\alpha)\right)-(-1)^{n} H_{n, m-1}\left(\delta_{n+1}^{0}(\alpha)\right)=\alpha-(-1)^{n m} \sigma_{n, m}^{*}(\alpha) .
$$

Let

$$
Z_{\mathbb{A} \times \mathbb{A}}^{p}(X, *)_{00} \stackrel{H}{\rightarrow} Z^{p}(X, *+1)_{0}, \quad \mathscr{H}_{\mathbb{A} \times \mathbb{A}}^{p}(X, *)_{00} \stackrel{H}{\rightarrow} \mathscr{H}^{p}(X, *+1)_{0},
$$

be the maps which are $H_{n, m}$ on the $(n, m)$-component. Let

$$
\mathscr{D}_{\mathbb{A} \times \mathbb{A}, Z^{p}}^{2 p-*}(X, p)_{00} \stackrel{H}{\rightarrow} \mathscr{D}_{\mathbb{A}, Z^{p}}^{2 p-*-1}(X, p)_{0},
$$


be the maps which are $(-1)^{r} H_{n, m}$ on the $(r,-n,-m)$-component. Observe that now

$$
d_{\mathscr{D}} H=-H d_{\mathscr{D}} .
$$

Let

$$
H: \widehat{Z}_{\mathbb{A} \times \mathbb{A}}^{p}(X, n)_{00} \rightarrow \widehat{Z}^{p}(X, n+1)_{0}
$$

be defined by

$$
H\left(Z, \alpha_{0}, \alpha_{1}, \alpha_{2}, \alpha_{3}\right)=\left(H(Z), H\left(\alpha_{0}\right), \alpha_{1},-H\left(\alpha_{2}\right),-H\left(\alpha_{3}\right)\right) .
$$

Let $x=\left(Z, \alpha_{0}, \alpha_{1}, \alpha_{2}, \alpha_{3}\right) \in \widehat{Z}_{\mathbb{A} \times \mathbb{A}}^{p}(X, n)_{00}$. Then

$$
\begin{aligned}
d H(x)= & \left(\delta H(Z), d_{s} H\left(\alpha_{0}\right), d_{\mathscr{D}}\left(\alpha_{1}\right), \gamma_{1} H(Z)-\gamma_{1}^{\prime} H\left(\alpha_{0}\right)\right. \\
& \left.+\delta H\left(\alpha_{2}\right), \rho H\left(\alpha_{0}\right)+d_{S} H\left(\alpha_{3}\right)-\alpha_{1}\right) \\
H d(x)=( & H \delta(Z), H d_{S}\left(\alpha_{0}\right), d_{D}\left(\alpha_{1}\right),-H \gamma_{1}(Z)+H \gamma_{1}^{\prime}\left(\alpha_{0}\right)+H \delta\left(\alpha_{2}\right), \\
& \left.-H \rho\left(\alpha_{0}\right)+H d_{S}\left(\alpha_{3}\right)+H\left(\alpha_{1}\right)\right) .
\end{aligned}
$$

Observe that for $\alpha_{0} \in \tau \mathcal{D}_{\log , Z^{p}}^{r}\left(X \times \square^{n} \times \square^{m}, p\right)_{00}$, we have

$$
\begin{aligned}
& H d_{s}\left(\alpha_{0}\right)=H d_{D}\left(\alpha_{0}\right)+(-1)^{r} H \delta\left(\alpha_{0}\right)=-d_{\mathscr{D}} H\left(\alpha_{0}\right)+(-1)^{r} H \delta\left(\alpha_{0}\right), \\
& d_{s} H\left(\alpha_{0}\right)=d_{\mathscr{D}} H\left(\alpha_{0}\right)+(-1)^{r} \delta H\left(\alpha_{0}\right) .
\end{aligned}
$$

The same remark applies to $\alpha_{3} \in \tau \mathscr{D}_{\log }^{r}\left(X \times \square^{n} \times \square^{m}, p\right)_{00}$. Moreover, since $\alpha_{1}$ equals zero in all degrees but 0 and $H$ is the identity in degree zero, we have, by Lemma 5.37,

$$
d H(x)+H d(x)=x-\sigma_{\square}^{*}(x) .
$$

Corollary 5.38. The following diagram is commutative up to homotopy:

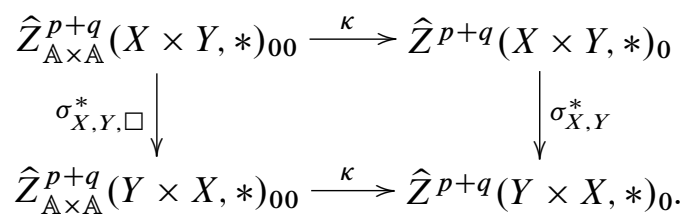

Proof. It follows from Proposition 5.35.

Corollary 5.39. Let $X, Y$ be arithmetic varieties.

(i) Under the canonical isomorphism $X \times Y \cong Y \times X$, the pairing

$$
\widehat{\mathrm{CH}}^{p}(X, n) \otimes \widehat{\mathrm{CH}}^{q}(Y, m) \stackrel{\cup}{\rightarrow} \widehat{\mathrm{CH}}^{p+q}(X \times Y, n+m),
$$

is graded commutative with respect to the degree $n$.

(ii) The internal pairing

$$
\widehat{\mathrm{CH}}^{p}(X, n) \otimes \widehat{\mathrm{CH}}^{q}(X, m) \stackrel{\cup}{\rightarrow} \widehat{\mathrm{CH}}^{p+q}(X, n+m),
$$

is graded commutative with respect to the degree $n$. 
5.5. Associativity. We prove here that the product for the higher arithmetic Chow groups is associative. First of all, observe that the product on $Z^{*}(X, *)_{0}$ is strictly associative. Hence, all that remains is to study the associativity of the product in the complexes with differential forms, except for $Z D_{\log }^{2 p}(X, p)_{*}$, where it is already associative. The key point will be Proposition 1.26.

Denote by $h$ the homotopy for the associativity of the product in the Deligne complex of differential forms of Proposition 1.26. Let $X, Y, Z$ be complex algebraic manifolds. Then the external product $\bullet_{\mathbb{A}}$ is associative, in the sense that there is a commutative diagram up to homotopy:

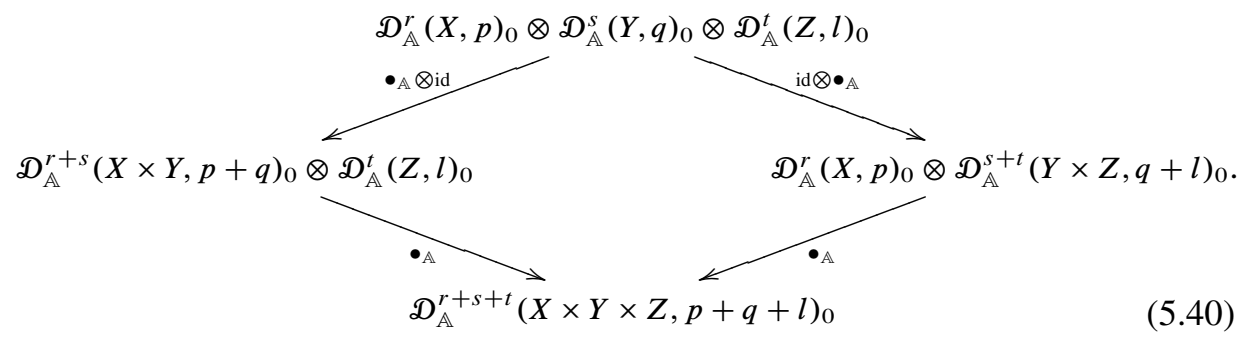

This follows from the fact that the homotopy $h$ is functorial (see [5]).

Proposition 5.41. Let $X, Y, Z$ be complex algebraic manifolds. Then there is a commutative diagram, up to homotopy:

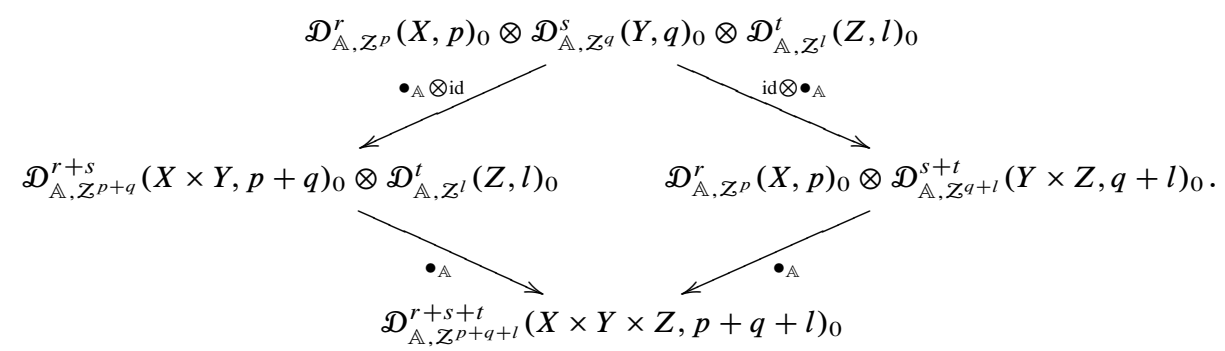

Proof. In order to prove the proposition, we need to introduce some new complexes, which are analogous to $s\left(i_{X, Y}^{p, q}\right)^{*}$, but with the three varieties $X, Y, Z$. Due to the similarity, we will leave the details to the reader.

$$
\begin{aligned}
& \text { We write } \square_{X, Y, Z}^{n, m, d}=X \times Y \times Z \times \square^{n+m+d} \text {. Let } \\
& A^{*}=\mathscr{D}_{\log }^{*}\left(\square_{X, Y, Z}^{n, m, d} \backslash \mathcal{Z}_{X, n}^{p}, k\right) \oplus \mathcal{D}_{\log }^{*}\left(\square_{X, Y, Z}^{n, m, d} \backslash \mathcal{Z}_{Y, m}^{q}, k\right) \oplus \mathcal{D}_{\log }^{*}\left(\square_{X, Y, Z}^{n, m, d} \backslash Z_{Z, d}^{l}, k\right),
\end{aligned}
$$

and

$$
\begin{aligned}
& B^{*}= \mathcal{D}_{\log }^{*}\left(\square_{X, Y, Z}^{n, m, d} \backslash \mathcal{Z}_{X, Y, n, m}^{p, q}, k\right) \oplus \mathcal{D}_{\log }^{*}\left(\square_{X, Y, Z}^{n, m, d} \backslash \mathcal{Z}_{X, Z, n, d}^{p, l}, k\right) \\
& \oplus \mathcal{D}_{\log }^{*}\left(\square_{X, Y, Z}^{n, m, d} \backslash Z_{Y, Z, m, d}^{q, l}, k\right),
\end{aligned}
$$


and consider the sequence of morphisms of complexes

$$
A^{*} \stackrel{i}{\rightarrow} B^{*} \stackrel{j}{\rightarrow} \mathscr{D}_{\log }^{*}\left(\square^{n, m, d} \backslash \mathcal{Z}_{X, Y, Z}^{p, q, l}, k\right) .
$$

By analogy with the definition of $s\left(-j_{X, Y}^{p, q}(n, m)\right)^{*}$, denote by $s\left(-j_{X, Y, Z}^{p, q, l}(n, m, d)\right)^{*}$ the simple complex associated to this sequence of morphisms. Consider the morphism

$$
\begin{aligned}
& \mathscr{D}_{\log }^{*}\left(\square_{X, Y, Z}^{n, m, d}, k\right) \stackrel{i_{X, Y, Z}^{p, q, l}(n, m, d)}{\longrightarrow} s\left(-j_{X, Y, Z}^{p, q, l}(n, m, d)\right)^{*}, \\
& \omega \quad \longmapsto \quad(\omega, \omega, \omega, 0,0,0,0) .
\end{aligned}
$$

Observe that for every $n, m, d$, the simple of this morphism is a cochain complex. Moreover, considering the normalized complex associated to the cubical structure at every component of $s\left(i_{X, Y, Z}^{p, q, l}(\cdot, \cdot, \cdot)\right)^{*}$, we obtain the cochain complex $s\left(i_{X, Y, Z}^{p, q, l}\right)_{0}^{*}$ (analogous to the construction of $s\left(i_{X, Y}^{p, q}\right)_{0}^{*}$ in Remark 5.7).

Let $\mathscr{D}_{\mathbb{A} \times \mathbb{A} \times \mathbb{A}, Z_{X, Y, Z}^{p, q, l}}^{*}(X \times Y \times Z, p+q+l)_{0}$ be the complex analogous to $\mathscr{D}_{\mathbb{A} \times \mathbb{A}, Z_{X, Y}^{p, q}}^{*}(X \times Y, p+q)_{0}$, but with the cartesian product of 3 varieties. It is the simple complex associated to the analogous 4-iterated complex (see Remark 5.7).

Observe that there is a quasi-isomorphism

$$
\mathcal{D}_{\mathbb{A} \times \mathbb{A} \times \mathbb{A}, Z_{X, Y, Z}^{p, q, l}}^{*}(X \times Y \times Z, p+q+l)_{0} \stackrel{\sim}{\rightarrow} s\left(i_{X, Y, Z}^{p, q, l}\right)_{0}^{*} .
$$

We define a pairing

$$
s\left(i_{X, Y}^{p, q}(n, m)\right)_{0}^{r} \otimes \mathscr{D}_{\mathbb{A}, Z^{l}}^{s, d}(Z, l)_{0} \stackrel{\bullet}{\rightarrow} s\left(i_{X, Y, Z}^{p, q, l}(n, m, d)\right)_{0}^{r+s}
$$

by

$$
\begin{aligned}
(a,(b, c), d) \bullet\left(a^{\prime}, b^{\prime}\right)= & (-1)^{(n+m) s}\left(a \bullet a^{\prime},\left(b \bullet a^{\prime}, c \bullet a^{\prime},(-1)^{r} a \bullet b^{\prime}\right),\right. \\
& \left.\left(d \bullet a^{\prime},(-1)^{r-1} b \bullet b^{\prime},(-1)^{r-1} c \bullet b^{\prime}\right),(-1)^{r-2} d \bullet b^{\prime}\right) .
\end{aligned}
$$

Define analogously a pairing

$$
\mathcal{D}_{\mathbb{A}, Z^{p}}^{r, n}(X, p)_{0} \otimes s\left(i_{Y, Z}^{q, l}(m, d)\right)_{0}^{s} \stackrel{\bullet}{\rightarrow} s\left(i_{X, Y, Z}^{p, q, l}(n, m, d)\right)_{0}^{r+s}
$$

by

$$
\begin{aligned}
(a, b) \bullet\left(a^{\prime},\left(b^{\prime}, c^{\prime}\right), d^{\prime}\right)= & (-1)^{n s}\left(a \bullet a^{\prime},\left(b \bullet a^{\prime},(-1)^{r} a \bullet b^{\prime},(-1)^{r} a \bullet c^{\prime}\right),\right. \\
& \left.\left((-1)^{r-1} b \bullet b^{\prime},(-1)^{r-1} b \bullet c^{\prime}, a \bullet d^{\prime}\right), b \bullet d^{\prime}\right) .
\end{aligned}
$$

It is easy to check that these two morphisms are chain morphisms. 
Lemma 5.42. The diagram

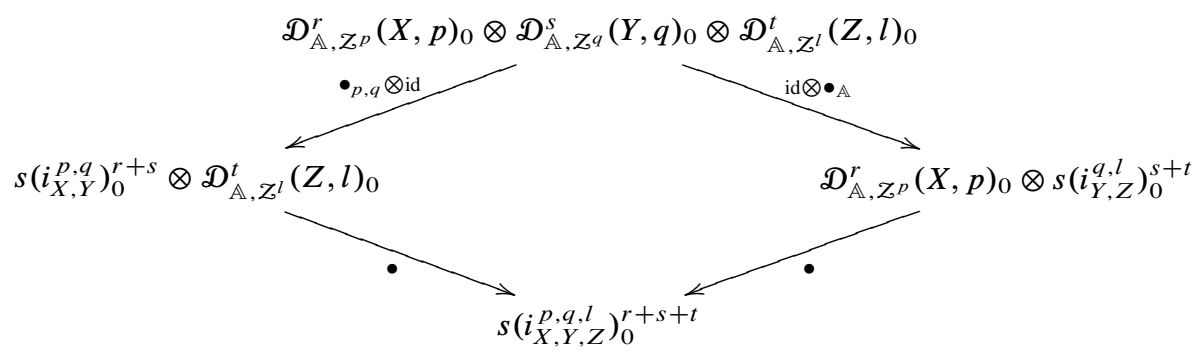

is commutative up to homotopy.

Proof. Let $\left(\omega_{1}, g_{1}\right) \in \tau \mathcal{D}_{\log , Z^{p}}^{r}\left(X \times \square^{n}, p\right)_{0},\left(\omega_{2}, g_{2}\right) \in \tau \mathscr{D}_{\log , Z^{q}}^{s}\left(Y \times \square^{m}, q\right)_{0}$, and $\left(\omega_{3}, g_{3}\right) \in \tau \mathscr{D}_{\log , Z^{l}}^{t}\left(Z \times \square^{d}, l\right)_{0}$. Then the composition of the morphisms on the left side of the diagram is

$$
\begin{array}{r}
(-1)^{(n+m) t+n s}\left(\left(\omega_{1} \bullet \omega_{2}\right) \bullet \omega_{3},\left(\left(g_{1} \bullet \omega_{2}\right) \bullet \omega_{3},(-1)^{r}\left(\omega_{1} \bullet g_{2}\right) \bullet \omega_{3},\right.\right. \\
\left.(-1)^{r+s}\left(\omega_{1} \bullet \omega_{2}\right) \bullet g_{3}\right),\left((-1)^{r-1}\left(g_{1} \bullet g_{2}\right) \bullet \omega_{3},(-1)^{r+s-1}\left(g_{1} \bullet \omega_{2}\right) \bullet g_{3},\right. \\
\left.\left.(-1)^{s-1}\left(\omega_{1} \bullet g_{2}\right) \bullet g_{3}\right),(-1)^{s-1}\left(g_{1} \bullet g_{2}\right) \bullet g_{3}\right) .
\end{array}
$$

The composition of the morphisms on the right side of the diagram is

$$
\begin{array}{r}
(-1)^{(n+m) t+n s}\left(\omega_{1} \bullet\left(\omega_{2} \bullet \omega_{3}\right),\left(g_{1} \bullet\left(\omega_{2} \bullet \omega_{3}\right),(-1)^{r} \omega_{1} \bullet\left(g_{2} \bullet \omega_{3}\right),\right.\right. \\
\left.(-1)^{r+s} \omega_{1} \bullet\left(\omega_{2} \bullet g_{3}\right)\right),\left((-1)^{r-1} g_{1} \bullet\left(g_{2} \bullet \omega_{3}\right),(-1)^{r+s-1} g_{1} \bullet\left(\omega_{2} \bullet g_{3}\right),\right. \\
\left.\left.(-1)^{s-1} \omega_{1} \bullet\left(g_{2} \bullet g_{3}\right)\right),(-1)^{s-1} g_{1} \bullet\left(g_{2} \bullet g_{3}\right)\right) .
\end{array}
$$

Then the homotopy for the commutativity of the diagram is given by

$$
\begin{aligned}
H_{n, m, d}=(-1)^{(n+m) t+n s}( & \left(h\left(\omega_{1} \otimes \omega_{2} \otimes \omega_{3}\right), h\left(g_{1} \otimes \omega_{2} \otimes \omega_{3}\right),\right. \\
& \left.(-1)^{r} h\left(\omega_{1} \otimes g_{2} \otimes \omega_{3}\right),(-1)^{r+s} h\left(\omega_{1} \otimes \omega_{2} \otimes g_{3}\right)\right), \\
& \left((-1)^{r-1} h\left(g_{1} \otimes g_{2} \otimes \omega_{3}\right),(-1)^{r+s-1} h\left(g_{1} \otimes \omega_{2} \otimes g_{3}\right),\right. \\
& \left.\left.(-1)^{s-1} h\left(\omega_{1} \otimes g_{2} \otimes g_{3}\right)\right),(-1)^{s-1} h\left(g_{1} \otimes g_{2} \otimes g_{3}\right)\right) .
\end{aligned}
$$

Observe that it gives indeed a homotopy, since $H$ and $\delta$ commute. 
Finally, the claim of Proposition 5.41 follows from the commutative diagram (all squares and triangles, apart from the one marked with \# are strictly commutative),

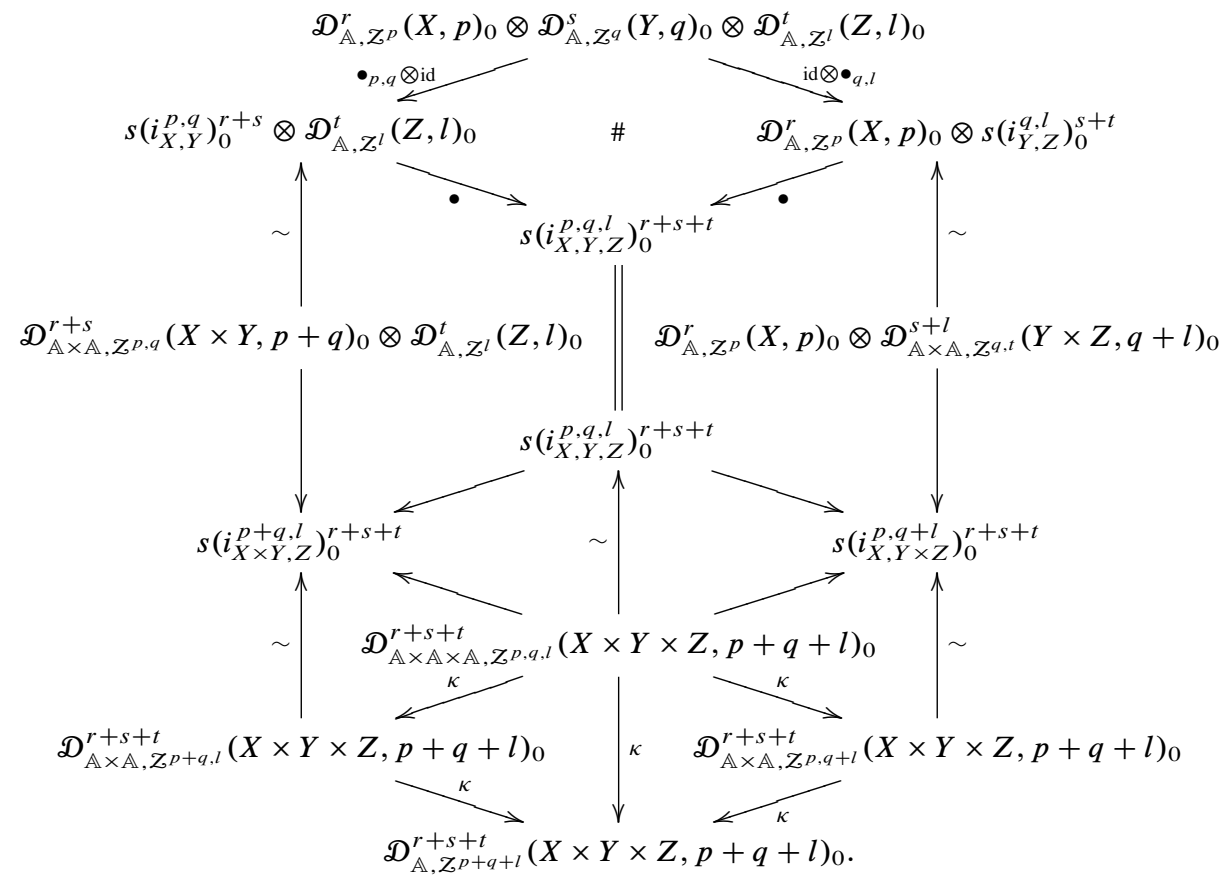

Remark 5.44. Observe that the homotopy constructed in the proof of Proposition 5.41 has no component in maximal degree, that is, in $\mathscr{D}_{\mathbb{A}, Z^{p+q+l}}^{2 p+2 q+2 l}(X \times Y \times Z, p+q+l)_{0}$.

Corollary 5.45. Let $X, Y, Z$ be arithmetic varieties.

(i) Under the canonical isomorphism $(X \times Y) \times Z \cong X \times(Y \times Z)$, the external pairing

$$
\widehat{\mathrm{CH}}^{p}(*, n) \otimes \widehat{\mathrm{CH}}^{q}(*, m) \otimes \stackrel{\cup}{\rightarrow} \widehat{\mathrm{CH}}^{p+q}(* \times *, n+m),
$$

is associative.

(ii) The internal pairing

$$
\widehat{\mathrm{CH}}^{p}(X, n) \otimes \widehat{\mathrm{CH}}^{q}(X, m) \stackrel{\cup}{\rightarrow} \widehat{\mathrm{CH}}^{p+q}(X, n+m),
$$

is associative.

Proof. It follows from (5.40) and Proposition 5.41, together with Remark 5.44 and the compatibility of the homotopies in (5.40) and Proposition 5.41. For $n=m=l=0$, the associativity follows from equality (1.27). 
Finally, we have proved the following theorem.

Theorem 5.46. Let $X$ be an arithmetic variety over an arithmetic field $K$. Then

$$
\widehat{\mathrm{CH}}^{*}(X, *):=\bigoplus_{p \geq 0, n \geq 0} \widehat{\mathrm{CH}}^{p}(X, n)
$$

is a commutative and associative ring with unity (graded commutative with respect to the degree $n$ and commutative with respect to the degree $p$ ). Moreover, the morphism $\widehat{\mathrm{CH}}^{*}(X, *) \stackrel{\zeta}{\rightarrow} \mathrm{CH}^{*}(X, *)$ of Proposition 4.4 is a ring morphism.

\section{References}

[1] A. A. Berlinson, Notes on absolute Hodge cohomology. In Applications of algebraic $K$-theory to algebraic geometry and number theory, Part I, II (Boulder, Colo., 1983), Contemp. Math. 55, Amer. Math. Soc., Providence, RI, 1986, 35-68. Zbl 0621.14011 MR 0923132

[2] S. Bloch, Some notes on elementary properties of higher chow groups, including functoriality properties and cubical chow groups.

http://www.math.uchicago.edu/ bloch/publications.html

[3] S. Bloch, Algebraic cycles and higher K-theory. Adv. in Math. 61 (1986), no. 3, 267-304. Zbl 0608.14004 MR 0852815

[4] S. Bloch, Algebraic cycles and the Berllinson conjectures. In The Lefschetz centennial conference (Mexico City, 1984), Part I, Contemp. Math. 58, Amer. Math. Soc., Providence, RI, 1986, 65-79. Zbl 0605.14017 MR 0860404

[5] J. I. Burgos Gil, Arithmetic Chow rings and Deligne-Beilinson cohomology. J. Algebraic Geom. 6 (1997), 335-377. Zbl 0922.14002 MR 1489119

[6] J. I. Burgos Gil, E. Feliu and Y. Takeda, On Goncharov's regulator and higher arithmetic Chow groups. Internat. Math. Res. Notices 2011 (2011), no. 1, 40-73 Zbl 05841662 MR 2755482

[7] J. I. Burgos Gil, J. Kramer and U. Kühn, Arithmetic characteristic classes of automorphic vector bundles. Doc. Math. 10 (2005), 619-716. Zbl 1080.14028 MR 2218402

[8] J. I. Burgos Gil, J. Kramer and U. Kühn, Cohomological arithmetic Chow rings. J. Inst. Math. Jussieu 6 (2007), no. 1, 1-172. Zbl 1115.14013 MR 2285241

[9] J. I. Burgos Gil and S. Wang, Higher Bott-Chern forms and Beilinson's regulator. Invent. Math. 132 (1998), no. 2, 261-305. Zbl 0924.14005 MR 1621424

[10] P. Deligne, Théorie de Hodge. II. Inst. Hautes Études Sci. Publ. Math. 40 (1971), 5-57. Zbl 0219.14007 MR 0498551

[11] P. Deligne, Théorie de Hodge. III. Inst. Hautes Études Sci. Publ. Math. 44 (1974), 5-77. Zbl 0237.14003 MR 0498552

[12] A. Dold and D. Puppe, Homologie nicht-additiver Funktoren. Anwendungen. Ann. Inst. Fourier Grenoble 11 (1961), 201-312. Zbl 0098.36005 MR 0150183 
[13] H. Gillet, Riemann-Roch theorems for higher algebraic K-theory. Adv. in Math. 40 (1981), no. 3, 203-289. Zbl 0478.14010 MR 0624666

[14] H. Gillet and C. Soulé, Arithmetic intersection theory. Inst. Hautes Études Sci. Publ. Math. (1990), no. 72, 93-174 (1991). Zbl 0741.14012 MR 1087394

[15] H. Gillet and C. Soulé, Filtrations on higher algebraic K-theory. In Algebraic K-theory (Seattle, WA, 1997), Proc. Sympos. Pure Math. 67, Amer. Math. Soc., Providence, RI, 1999, 89-148. Zbl 0951.19003 MR 1743238

[16] A. B. Goncharov, Polylogarithms, regulators, and Arakelov motivic complexes. J. Amer. Math. Soc. 18 (2005), no. 1, 1-60. Zbl 1104.11036 MR 2114816

[17] A. Grothendieck, La théorie des classes de Chern. Bull. Soc. Math. France 86 (1958), 137-154. Zbl 0091.33201 MR 0116023

[18] A. Krishna and M. Levine, Additive higher Chow groups of schemes. J. Reine Angew. Math. 619 (2008), 75-140. Zbl 1158.14009 MR 2414948

[19] M. Levine, Bloch's higher Chow groups revisited. In K-theory (Strasbourg, 1992), Astérisque 226 (1994), 10, 235-320. Zbl 0817.19004 MR 1317122

[20] M. Levine, Mixed motives. Math. Surveys Monogr. 57, Amer. Math. Soc., Providence, RI, 1998. Zbl 0902.14003 MR 1623774

[21] M. Levine, Chow's moving lemma and the homotopy coniveau tower. K-Theory 37 (2006), no. 1-2, 129-209. Zbl 1117.19003 MR 2274672

[22] J. Milnor and J. Stasheff, Characteristic classes. Ann. of Math. Stud. 76, Princeton University Press, Princeton, NJ, 1974. Zbl 0298.57008 MR 0440554

[23] D. Quillen, Higher algebraic K-theory. I. In Algebraic K-theory, I: Higher K-theories (Proc. Conf., Battelle Memorial Inst., Seattle, Wash., 1972), Lecture Notes in Math. 341, Springer-Verlag, Berlin, 1973, 85-147. Zbl 0292.18004 MR 0338129

Received July 29, 2009

José Ignacio Burgos Gil, Instituto de Ciencias Matemáticas ICMAT (CSIC, UAM, UCM, UC3), 28049 Madrid, Spain

E-mail: burgos@icmat.es, jiburgosgil@gmail.com

Elisenda Feliu, Facultat de Matemàtiques, Universitat de Barcelona, 08007 Barcelona, Spain; current address: Department of Mathematics, University of Copenhagen, 2100 Copenhagen $\varnothing$, Denmark

E-mail: efeliu@math.ku.dk 